\title{
Microstructural Characterization of Dislocation Networks During Harper-Dorn Creep of fcc, bcc, and hep Metals and Alloys
}

\author{
Final Report \\ By \\ Marek A. Przystupa \\ Marek A. Przystupa, Principal Investigator \\ Alan J. Ardell, Co-Principal Investigator \\ Department of Materials Science and Engineering \\ University of California \\ Los Angeles, CA 90095-1595
}

Grant \# DE-FG02-03ER46077

Decmber 2007 
Summary

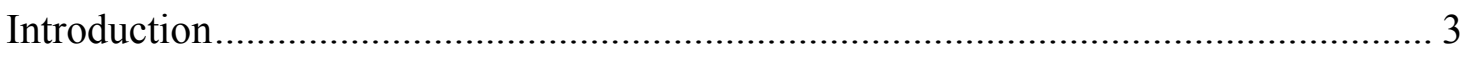

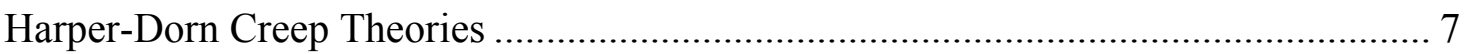

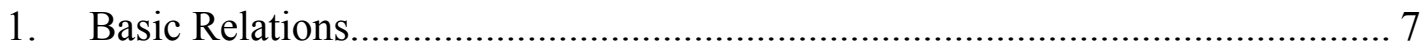

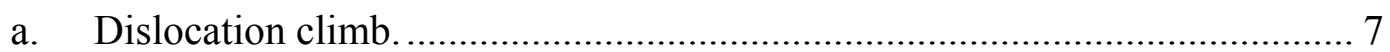

i. Climb of jogged edge dislocation. ........................................................... 9

ii. Climb of edge dislocation under supersaturated conditions. ........................ 11

b. Glide of jogged screw dislocation............................................................... 12

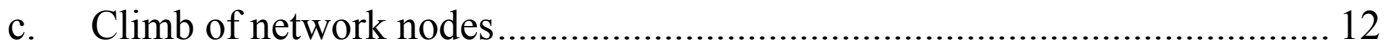

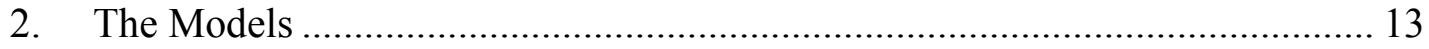

a. Harper-Shepard-Dorn jogged screw dislocation theory ................................ 14

b. Barrett-Muehleisen-Nix edge dislocation climb theory ................................. 16

c. Climb of jogged edge dislocations......................................................... 19

d. Climb of edge dislocations under supersaturated conditions........................ 20

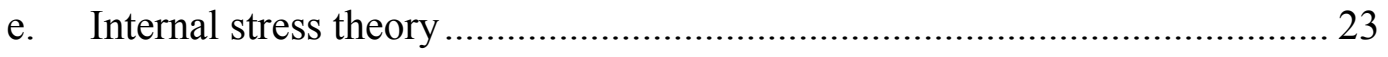

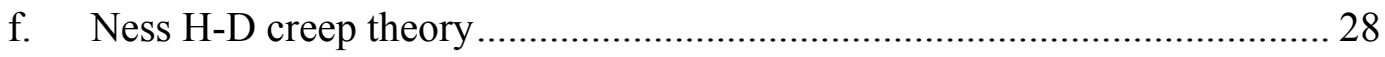

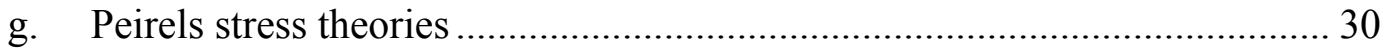

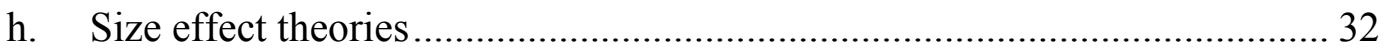

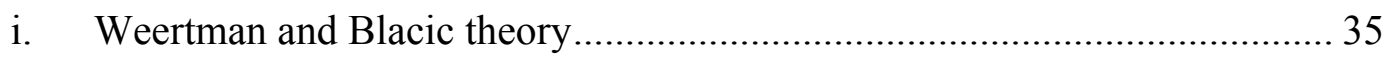

j. Intermediate temperature H-D creep theory ……………………………...... 37

The Dislocation Network Theory ............................................................................. 40

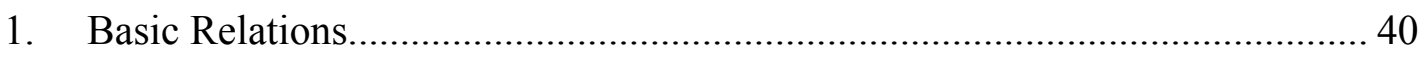

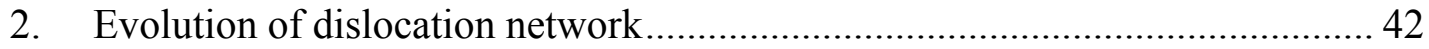

a. Network Refinement and Recovery Processes ............................................ 42

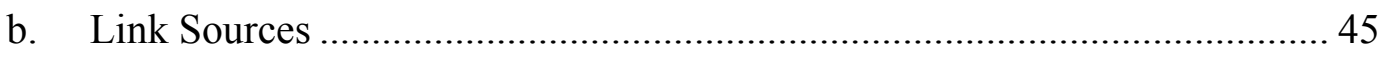

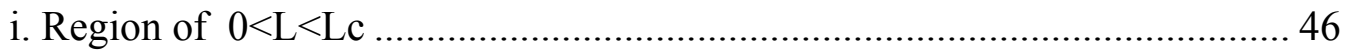

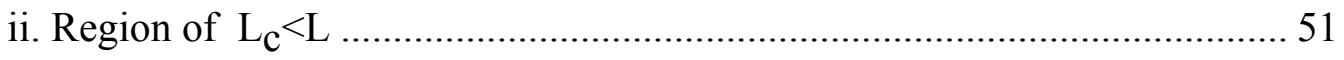

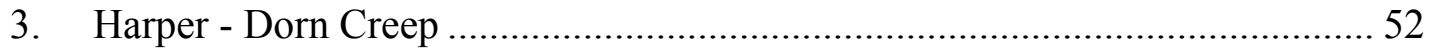

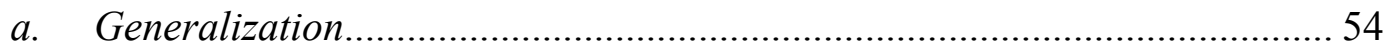

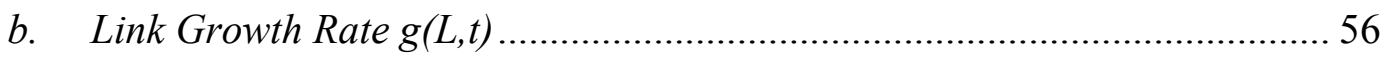




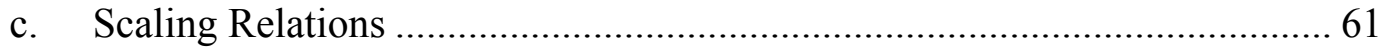

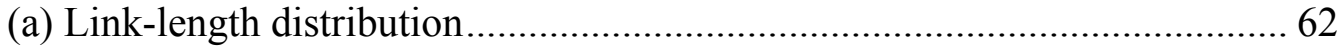

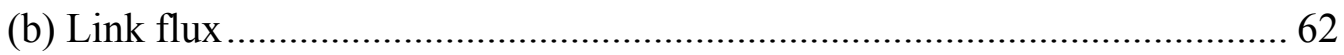

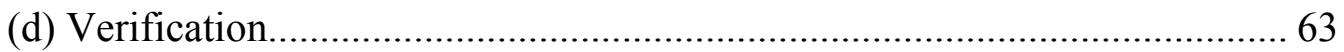

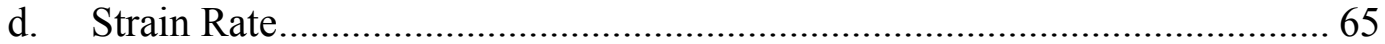

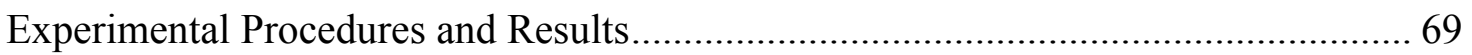

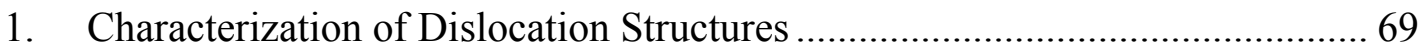

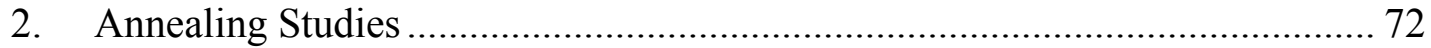

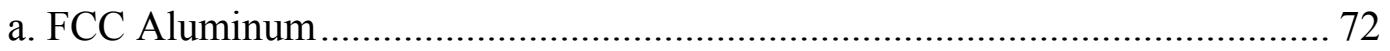

i. Samples pr-deformed at $913 \mathrm{~K}$........................................................... 74

ii. Samples pr-deformed at $620 \mathrm{~K}$............................................................. 77

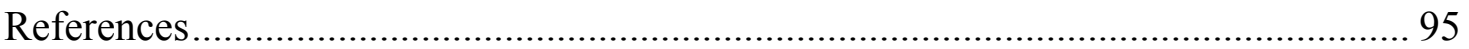

Appendix 1. Publications, Presentations and Personnel ....................................... 100

Appendix 2. Compilation of useful relationships ............................................... 102 


\section{$\underline{\text { Summary }}$}

Harper-Dorn (H-D) creep is observed in metals and geological materials exposed to very low stresses at temperatures close to the melting point. It is one of several types of creep processes wherein the steady-state strain rate is proportional to the applied stress, Nabarro-Herring creep and Coble creep being two other important processes. H-D creep can be somewhat insidious because the creep rates are much larger than those expected for Nabarro-Herring or Coble creep. Since the working conditions of structural components of power plants and propulsion systems, as well as the motion of the earth's mantle all involve very low stresses, an understanding of the factors controlling H-D creep is critical in preventing failures associated with those higher-than-expected creep rates. The purpose of this investigation was to obtain missing microstructural information on the evolution of the dislocation structures during static annealing of materials with fcc, bcc and hcp structure and use obtained results to test predictive capabilities of the dislocation network theory of H-D creep. In our view the evolutionary processes during static annealing and during Harper-Dorn creep are intimately related. The materials used in this study were fcc aluminum, hep zinc and bcc tin. All characterizations of dislocation structures, densities and dislocation link length distributions were carried out using the etch pit method. To obtain quantitative information on the evolution of the dislocation networks during annealing the pure fcc aluminum samples were pre-deformed by creep at 913 and $620 \mathrm{~K}$ and then annealed. The higher deformation temperature was selected to generate starting dislocation networks similar to those forming during Harper-Dorn creep and the lower, to obtain higher dislocation densities suitable for reliable estimates of the parameters of the network growth law. The measured experimental link length distribution were, after scaling, (1) the same for all annealing temperatures, (2) time invariant and (3) identical to the distributions obtained previously for Harper-Dorn creep. This has never been shown before and confirms our theoretical expectations that evolution of the dislocation networks during annealing and H-D creep is governed by the same growth law. Obtained results were also used to predict H-D steady creep rates from annealing kinetics data using equations of the dislocation network theory. For the three considered stresses the theory predicts systematically smaller creep rates by the average 
factor of 4.5. Considering that the creep rates have been predicted from the annealing data alone and without any adjustable parameters, this results shout be considered as outstanding. In case of hcp zinc the samples were pre-deformed in compression at constant stress of $4 \mathrm{MPa}$ at temperature of $573 \mathrm{~K}$ and subsequently annealed at the same temperature. During annealing samples readily recrystallized, but it was possible to obtain information on the link length distributions from several unrecrystallized grains. The results showed that the scaled link length distributions were time invariant and similar to those of the aluminum. The annealing studies on bec tin were also curtailed by the concurrent recrystallization. It was only possible to obtain link length distribution for samples deformed in compression at constant load of $2 \mathrm{MPa}$ at $423 \mathrm{~K}$ after unloading. The link length distribution was also in this case similar to that of the aluminum and zinc. These results suggest that the scaled link length distribution is universal and the same for the three considered crystal structures. This supports theoretical findings of these studies that appropriately scaled dislocation link length distribution should both universal and time invariant. We have also investigated the possibility of using alternative methods of estimating local dislocation densities from etch pits which could give more precise estimates of the dislocation link-lengths. The two most promising method are based on the use of Voronoi diagrams and uniform 3-connected nets. Both methods require further testing before they can be used in confidence in the analysis of the etch pit results. 


\section{$\underline{\text { Introduction }}$}

The phenomenon of Harper-Dorn (H-D) creep was discovered in the late fifties during studies of the creep of pure aluminum single and polycrystals (Harper and Dorn, 1957). This type of creep takes place at very low stresses and temperatures close to the melting point and manifests itself with unusually high creep rates that are three or more orders of magnitude faster than those expected for the diffusional or power-law creep under the same conditions. This accelerated creep behavior is important both from the technological and the scientific points of view. Technologically, its understanding is essential for improvements in the design of more reliable components in power plants and propulsion systems. For materials scientists, better understanding of the mechanisms controlling H-D creep will lead to advances in microstructural models for the transitions between various creep regimes (e.g. power-law creep and Nabarro-Herring or HarperDorn creep), the relationship between annealing and static recovery processes under stress-free condition and under applied stress, evolution of the dislocation network structure, and may eventually clarify the role of the Peierls stress in the very low-stress regime (Nabarro, 1997). H-D creep is also important in the earth sciences because olivine rocks, which are a major constituent of the earth's mantle, are exposed to stresses and temperatures corresponding to those in the H-D creep regime (Kohlstedt et al, 1974, Beron et al, 1996). A better understanding of H-D creep behavior will lead to the improvement of rheological models of the flow of the earth's mantle.

Although during the past forty years there have been numerous studies addressing H-D creep behavior, there is still no consensus regarding our understanding of this phenomenon, and particularly its microstructural characteristics (Kassner et al, 2007, Kumar et al, 2007). Nevertheless, the general characteristics of H-D creep behavior are more-or-less agreed upon (Yavari et al, 1982, Nabarro, 1989, Cadek et al, 1988, Mohamed et al, 1991, Kassner et al, 2000):

(i) The activation energy for H-D creep is equal to that for self-diffusion (Harper et al, 1957, Barrett et al, 1972)

(ii) The steady-state creep strain rate is 
- three orders of magnitude or more faster than the rate expected for bulk or grain-boundary diffusion controlled creep under the same conditions (Nabarro, 1948, Herring, 1950)

- proportional to the stress to the power of one (Harper et al, 1957, Barrett et al, 1972)

- independent of the grain size (Harper et al, 1957, Barrett et al, 1972)

- the same for pure metals and solid solutions

(iii) The steady state dislocation density is

- independent of the stress (Barrett et al, 1972);

- the same in pure metals and solid solutions (Barrett et al, 1972);

(iv) The H-D creep is suppressed by

- presence of small grains (Novotny et al, 1985)

- presence of precipitates (Barrett et al, 1972)

- initially high dislocation density (Mohamed et al, 1982)

(v) The stress at which Harper-Dorn creep gives way to power-law creep increases with increasing alloy content

(vi) There is disagreement whether or not subgrains are formed during creep Yavari et al, 1982, Barrett et al, 1972).

Not all of the above characteristics have been observed in all of the materials and not all them are universally accepted. Most disagreements exist regarding the very existence of the H-D creep and the lack of the dependency of the steady-state dislocation density on stress. The detractors attributed the H-D creep behavior to the effect of the low-amplitude temperature cycling during testing, measurements made during primary rather than steady state creep, necking, grain sliding and size effect (Weertman et al, 1984, Blum et al, 1999, Ginter et al, 2001, Ness et al, 2002). These concerns were addressed by Nabarro $(1989,2000,2000$ a) who showed that they can be dismissed as unsubstantiated. There are also disagreements about theoretical interpretations of the H-D creep results. Most of the theories attribute high steady-state creep rates to diffusioncontrolled dislocation motion but they disagree on the mechanism responsible for that motion. The most common assumption is that dislocations are predominantly of edge character and that their climb is controlled by the diffusion of vacancies (Nabarro, 1989, 
Wang et al, 1994, 1996, Ruano et al, 1988, 1988a, 1992). During steady-state deformation the stresses on dislocations are balanced by either the Peierls stress or by internal stresses, and creep results from the thermally activated dislocation climb events which are biased in one direction by the external stress.

Theories invoking the Peierls stress or internal stress adequately describe the steady-state H-D creep rate, but they are incapable of describing the decreasing creep rate during the primary stage. The only theory compatible with the behavior in both regions is the dislocation network coarsening theory ( Lagneborg, 1972, Ardell et al, 1984 and 1986). In this approach H-D creep takes place as a result of the coarsening of the dislocation network, which is continuously refined by collisions between growing links. The network theory is also the only one capable of predicting not only the steady-state creep rates but also the entire H-D creep curve and other characteristics of H-D creep behavior (e.g. the distribution of dislocation link lengths) that other theories cannot.

The predictive capabilities of the network theory are currently limited only by the availability of the microstructural data necessary for its verification. Specifically, for metals the most extensive studies on H-D creep have been done on fcc aluminum and its solid solutions but even those results are incomplete (Harper et al, 1957, Barrett et al, 1972, Mohamed et al, 1982, Ginter et al, 2001, Ardell et al, 1986, Harper et al, 1958). They lack, in particular, data on the kinetics of static annealing of the dislocation network structure; this information is an essential ingredient of the network theory. Some of the other microstructural results are also contradictory; it has been reported that the dislocation density both increases and is independent of stress (Kohlstedt et al, 1974, Yavari et al, 1982, Barrett et al, 1972, Ardell et al, 1986, Nost et al, 1969); similar inconsistencies exist regarding the formation of subgrains (Yavari et al, 1982, Barrett et al, 1972, Mohamed et al, 1982). The frequently cited information on the types of dislocations forming networks is also not entirely reliable. As pointed out recently by Nes, 1998, these conclusions were based on the TEM characterizations of samples that had dislocation densities several orders of magnitude higher than those expected for H-D creep. Conclusions regarding jogs on dislocations have also been drawn from the examination of TEM micrographs (Yavari et al, 1982, Mohamed et al, 1982) which 
revealed dislocation densities far exceeding the small densities characteristic of H-D creep $\left(70-90 \mathrm{~mm}^{-2}\right)$.

For materials with other crystal structures the microstructural data are even more scarce. The standard H-D creep results on dislocation density and creep rates for bcc metals come from two different studies on $\mathrm{Fe}+3 \mathrm{Si}$ alloys, and from slightly different alloys tested using different procedures (Jones et al, 1966, Strang et al, 1973). For hcp metals there are number of sources of data on steady-state H-D creep rates but only semiquantitative information on the dislocation densities. One such investigation is that of Novotny et al., 1985) on hcp $\alpha-\mathrm{Zr}$ in which they state that “...the dislocation density is close to $10^{12} \mathrm{~m}^{-2}$ and does not increase in the course of creep at the highest stress considered..." This information is obviously not very precise. Other frequently quoted data on dislocation densities in hep $\alpha$-Ti and $\beta$-Co are taken not from measurements but from the best fit of a theory to data on the steady-state creep rates (Malakondaiah et al, 1979). There is also a large body of data on H-D creep rates and dislocation densities for geological materials but, unfortunately, it is also as incomplete as that for metals (Kohlstedt et al, 1974, Wang, 1996, Banerdt et al, 1985, Ramesh et al, 1986, 1986a, Justice, 1982).

It appears then that there is a need for obtaining a reliable and consistent set of $\mathrm{H}$ D creep microstructural data for the representative materials with the most important metallic crystal structures. These data could be then incorporated into the H-D creep microstructural database that could be used to provide critical tests of not only the dislocation network theory but also all other H-D creep models requiring microstructural input. The purpose of this investigation was to fill that need by (1) augmenting the existing $\mathrm{H}-\mathrm{D}$ creep results for $\mathrm{Al}$ with the missing data on the kinetics of static recovery of the dislocation microstructure, (2) generating static recovery data for representative materials with bcc and hcp crystal structures and (3) testing the predictive capabilities of the dislocation network model of H-D creep by using the annealing results to generate theoretical creep curves and comparing them with the creep curves obtained experimentally. The theoretical background behind the different H-D creep and H-D network theories, the experimental procedures used and the obtained results are described 
in more detail below. In addition, Appendix 1 contains list of publications, presentations and information on the personnel involved in this project.

\section{Harper-Dorn Creep Theories}

\section{Basic Relations}

The starting point of H-D creep theories is the Orowan's strain rate relation in the form:

$$
\dot{\varepsilon}=\alpha \rho b \mathrm{v}
$$

where $\alpha$ is geometrical constant, $\rho$ is the mobile dislocation density, b dislocations Burgers vector and $v$ is dislocation glide or climb velocity. What differentiate various theories are their assumptions regarding the mechanisms controlling the movement of dislocation, hence their velocities. During creep dislocations propagate by a series of glide and climb movements and the slower of the two controls the dislocation velocity hence the creep rate. In pure metals, with which we are primarily concern with, the glide mobility of the jogs-free dislocation is high, which means that the creep rate must be controlled by the slower diffusional processes such, climb, glide of the jogged dislocations, movement of network nodes, coarsening of the dislocation network etc. Before proceeding to the specific H-D creep theories it is then essential to consider factors controlling those processes.

\section{a. Dislocation climb.}

The diffusion controlled dislocation climb has been considered in details by Weertman 1968, Lothe 1960, Hirth et al. 1968, Friedel 1964. For the case of edge dislocation it has been determined that the external stress, $\sigma$, perpendicular to dislocation extra half-plane creates a climbing force per unit length of dislocation line, $\mathrm{F} / \mathrm{L}$, equal to

$$
\frac{F}{L}=\sigma b
$$

This force results in the change of the equilibrium concentration of vacancies at the dislocation line from the stress-free value of $\mathrm{c}_{\mathrm{o}}$ to 


$$
c=c_{o} \exp \left(\frac{\sigma \Omega}{k_{B} T}\right)
$$

where $\Omega$ is atomic volume. At the steady-state conditions the difference in vacancy concentration at dislocation and at the regions at distance $\mathrm{R}$, where vacancy concentration is maintained at $\mathrm{c}_{\mathrm{o}}$, results in the concentration profile described by relation:

$$
c(r)=c_{o}\left\{1-\frac{\ln (R / r)}{\ln (R / b)}\left[1-\exp \left(\frac{\sigma \Omega}{k_{B} T}\right)\right]\right\} \approx c_{o}\left\{1+\frac{\ln (R / r)}{\ln (R / b)} \frac{\sigma \Omega}{k_{B} T}\right\}
$$

where $\mathrm{r}$ is the distance from the dislocation and where it was assumed that $\sigma \Omega<<k T$. The net current of vacancies per unit length of dislocation line, $I$, away from dislocation is than equal to

$$
I=-2 \pi r D_{V} \frac{\partial c}{\partial r}=\frac{2 \pi D_{V} \sigma \Omega c_{o}}{k_{B} T \ln (R / b)}
$$

where $D_{V}$ is vacancy diffusion coefficient. The volume of vacancies removed per unit length and time is then $I \cdot \Omega$, which divided by Burgers vector gives climb velocity

$$
v_{c}=\frac{I \Omega}{b}=\frac{2 \pi D_{V} \sigma \Omega^{2} c_{o}}{b k_{B} T \ln (R / b)}
$$

Noting that atomic self-diffusion coefficient, $D_{s}$, is equal to

$$
D_{s}=D_{V} \Omega c_{o}
$$

gives the final expression for edge dislocation climb velocity equal to

$$
v_{c}=\frac{2 \pi D_{s} \Omega \sigma}{b k_{B} T \ln (R / b)}
$$

As shown by Hirth et al. 1968 (p. 512, eq. 15-90) this equation can be generalized and for an arbitrary stress tensor and mixed dislocation it has the form:

$$
v_{c}=\frac{2 \pi D_{s} \Omega\left(F_{e l} / L\right)}{b_{e}^{2} k_{B} T \ln (R / b)}
$$

where $b_{\mathrm{e}}$ is dislocation edge component and

$$
\frac{F_{e l}}{L}=\frac{(\mathbf{b} \cdot \boldsymbol{\sigma} \times \xi) \cdot(\mathbf{b} \times \xi)}{|\mathbf{b} \times \xi|}
$$

$\sigma$ is here the stress tensor and $\xi$ is a unit vector along dislocation line. Assuming $\Omega \sim \mathrm{b}^{3}$, and that $\ln (\mathrm{R} / \mathrm{b}) \sim 2 \pi$ gives standard form of the climb equation used in creep literature: 


$$
v_{c}=b \frac{D_{s} \sigma b}{k_{B} T}
$$

It is also important to note that during creep and annealing some of the dislocations will act as dislocation sinks and some as sources, hence the characteristic distance $\mathrm{R}$ can be assume equal to the average dislocation spacing, $\langle L\rangle$,

$$
R \approx\langle L\rangle=\frac{\beta}{\rho^{1 / 2}}
$$

where $b$ is geometrical constant of the order of one. Combining (1.8) and (1.12) gives then

$$
v_{c}=\frac{2 \pi D_{s} \Omega \sigma}{b k_{B} T \ln \left(\beta / b \rho^{1 / 2}\right)}
$$

The more specialized climb cases follow.

\section{i. Climb of jogged edge dislocation.}

Following treatment by Hirth et al 1968, an edge dislocation under stress $\sigma$ generates a force on the jog of height $h$ equal to

$$
F_{j}=\sigma b h
$$

The work to move the jog by one atomic distance, $a$, along dislocation by generating a vacancy is then

$$
W=F_{j} a=\sigma b h a \approx \sigma \Omega
$$

The resulting local equilibrium concentration of vacancies will be then the same as that given by eq. (1.3). However, since the jog can be treated as a point vacancy source, the local vacancy concentration profile changes; it has now spherical symmetry and is given by relation

$$
c(r)=c_{o}\left\{1+\frac{b}{r}\left(\exp \left[\frac{\sigma \Omega}{k_{b} T}\right]-1\right)\right\}
$$

which gives vacancy current

$$
I=-4 \pi r^{2} D_{V} \frac{\partial c}{\partial r}=4 \pi D_{V} b c_{o}\left(\exp \left[\frac{\sigma \Omega}{k_{b} T}\right]-1\right)
$$


and jog velocity along dislocation line equal to

$$
v_{j}=I a=4 \pi D_{V} b a c_{o}\left(\exp \left[\frac{\sigma \Omega}{k_{b} T}\right]-1\right) \approx \frac{4 \pi D_{s} \sigma b^{2}}{k_{B} T}
$$

The last part of the equation was obtained by incorporating eq. (1.7) and assuming $\sigma \Omega<<$ $k T$. Converting jog drift velocity to dislocation climb velocity, $\mathrm{v}_{\text {cej }}$, gives

$$
v_{c e j}=\frac{b}{l_{j c}} v_{j}=\frac{4 \pi b^{2}}{l_{j c}} \frac{D_{s} b \sigma}{k_{B} T}
$$

where $l_{j c}$ is the jog spacing.

When pipe diffusion along dislocation line is considerable, it is necessary to take into account the distance traveled by vacancy along dislocation core before diffusing to the matrix. According to Hirth et al 1968 the average value of this distance is

$$
\bar{z}=b \exp \left(\frac{Q_{s}-Q_{p}}{2 k_{b} T}\right)
$$

where $Q_{s}$ is activation energy for volume and $Q_{p}$ for core self-diffusion. Depending on the value of $\bar{z}$ it is possible to consider the following three cases:

- Case 1: If $\bar{z}<<l_{j c}$ each jog can be considered as a non-interacting line vacancy source of width $2 \bar{z}$. Eq. (1.18) and (1.19) need to be then modified for the change of source geometry which results in the expression for jog velocity in the form

$$
v_{j p}=\frac{4 \pi D_{s} \sigma b^{2}}{k_{B} T \ln (\bar{z} / b)} \exp \left(\frac{Q_{s}-Q_{p}}{2 k_{b} T}\right)
$$

Dislocation climb velocity becomes then equal to

$$
v_{c e j p}=\frac{4 \pi b^{2}}{l_{j c} \ln (\bar{z} / b)} \frac{D_{s} b \sigma}{k_{B} T} \exp \left(\frac{Q_{s}-Q_{p}}{2 k_{b} T}\right)
$$

- Case 2: If $\bar{z}>l_{j c}$ jogs act as a "continuous" source of vacancies with width equal to the entire dislocation length. This is equivalent to the case of diffusional climb of dislocation without jogs with climb velocity given by eqs (1.8) or (1.13).

- Case3: If $\bar{z} \sim l_{j c}$ the diffusional field of jogs overlap and the interaction of those fields result in the saturation with jog velocity independ on $\bar{z}$. As shown by Hirth et al 1968 the saturation takes place when 


$$
\bar{z}>l_{j c} \frac{\ln (\bar{z} / b)}{\ln \left(R / l_{j c}\right)}
$$

The jog velocity is in this case

$$
v_{j s}=\frac{2 \pi D_{s} \sigma b l_{j c}}{k_{B} T \ln \left(R / l_{j c}\right)}
$$

which gives dislocation climb velocity equal to

$$
v_{c e j s}=\frac{2 \pi D_{s} \sigma b^{2}}{k_{B} T \ln \left(R / l_{j c}\right)}
$$

It is important to point out that all equation in this section assume stationary vacancy concentration field. The refinements taking into account dislocation motion can be found in Nix et al 1971, Turunen et al 1973, 1974 and 1976.

\section{ii. Climb of edge dislocation under supersaturated conditions.}

Up until now it has been assumed that the concentration of vacancies at dislocation is not at equilibrium which produces a driving force to climb. This is not true when diffusion of vacancies produced by climb is sluggish. In this case concentration rises to the equilibrium level and climb stops. The climb velocity is in this case controlled not by the emission of vacancies by dislocation but by the diffusion of vacancies from dislocation. For this case the climb velocity is given by Hirth et al. 1968 as

$$
v_{c s s}=\frac{2 \pi D_{s}}{b \ln (R / b)}\left(\frac{c}{c_{o}}-1\right)
$$

The term in the brackets can be estimated from eq. (1.3) to be

$$
\ln \left(\frac{c}{c_{o}}\right) \approx \frac{c}{c_{o}}-1=\frac{\sigma \Omega}{k_{B} T}
$$

Combining eqs (1.12), (1.26) and (1.27) gives climb velocity

$$
v_{c s s}=\frac{2 \pi b}{\ln \left(\frac{\beta}{b \rho^{1 / 2}}\right)} \frac{D_{s} b \sigma}{k_{B} T}
$$

which, as expected, is the same as climb velocity of straight edge dislocation given be eq. (1.13). 


\section{b. Glide of jogged screw dislocation}

This case is similar to the one of bulk diffusion controlled climb of edge dislocation with jogs (Barrett and Nix 1965). The only difference is that the force on a jog is now

$$
F_{j}=\sigma l_{j} b
$$

which gives the work to advance each jog, hence also entire screw dislocation, by one atomic distance, $a$, equal to

$$
W=F_{j} a=\sigma l_{j} b a
$$

The jog drift velocity is in this case equivalent to the dislocation velocity, hence incorporating changes from eqs. (1.29) and (1.30) into eq. (1.18) gives dislocation glide velocity equal

$$
v_{g j}=\frac{4 \pi D_{s} \sigma b a l_{j}}{h k_{B} T} \approx 4 \pi l_{j} \frac{D_{s} \sigma b}{k_{B} T}
$$

As evident form eq. (1.18) the last equation is only valid in the small stress regime.

\section{c. Climb of network nodes}

As discussed by Friedel 1964 (p.109), Miekk-Oja et al 1972 and Evans and Knowles 1977, the movement of nodes requires formation of jogs on one or more dislocation joined at the node hence emission or absorption of vacancies. The node can be then treated similarly as a jog on an edge dislocation and its velocity described by modified eq. (1.18). Assuming that the average climbing force per unit length of dislocations forming jogs is $F$ gives the node velocity equal

$$
v_{n b}=\frac{4 \pi D_{s} F b}{k_{B} T}
$$

for bulk diffusion and

$$
v_{n p}=\frac{4 \pi D_{s} F b}{k_{B} T \ln (\bar{z} / b)} \exp \left(\frac{Q_{s}-Q_{p}}{2 k_{B} T}\right)
$$


for pipe diffusion controlled climb. In both cases the most important contributions to force $F$ are applied stress, elastic interactions between links and the line tension (Friedel 1964, Evans et al 1977).

\section{The Models}

The dislocation velocities from the previous sections will be now used to sort out various H-D creep theories. Wherever possible the obtained creep rates will be compared with the experimental data. The data included in comparisons are only those for which not only creep rates but also dislocation densities are available. The materials and sources of data are summarized in Table I.

Table 1. References used as the sources of data for verifying H-D creep models.

\begin{tabular}{|c|c|c|c|c|}
\hline Data Set & $\begin{array}{l}\text { Crystal } \\
\text { structure }\end{array}$ & $\begin{array}{c}\text { Strain rates and } \\
\text { stresses }\end{array}$ & Dislocation density & $\begin{array}{l}\text { Diffusion } \\
\text { coefficient }\end{array}$ \\
\hline $\mathrm{Al}$ (mono) & fcc & Lee, 1985 & Lee, 1985 & Frost et al, 1982 \\
\hline $\mathrm{Al}$ (poly) & fcc & Barrett et al, 1972 & Barrett et al, 1972 & Frost et al, 1982 \\
\hline $\mathrm{NaCl}$ & $\mathrm{fcc}$ & Banerdt et al, 1985 & Banerdt et al, 1985 & Frost et al, 1982 \\
\hline$\alpha-Z r$ & hcp & Novotny et al, 1985 & Novotny et al, 1985 & Sargent et al., 1982 \\
\hline $\mathrm{MgO}$ & cubic & Ramesh et al, 1986 & Ramesh et al, 1986a & Frost et al, 1982 \\
\hline $\mathrm{Fe}-3 \mathrm{Si}$ & bcc & Jones et al, 1966 & Stang, et al, 1973 & Frost et al, 1982 \\
\hline $\mathrm{Al}-5 \mathrm{Mg}$ & fcc & $\begin{array}{l}\text { Malakondaiah et al, } \\
1979\end{array}$ & $\begin{array}{l}\text { Malakondaiah et al, } \\
1979\end{array}$ & Frost et al, 1982 \\
\hline Quartz & hexagonal & Wang, 1996 & Wang, 1996 & Wang, 1996 \\
\hline$\alpha-\mathrm{Ti}$ & hcp & $\begin{array}{l}\text { Malakondaiah et al, } \\
1979\end{array}$ & $\begin{array}{l}\text { Malakondaiah et al, } \\
1979\end{array}$ & Frost et al, 1982 \\
\hline$\beta-\mathrm{Co}$ & fcc & $\begin{array}{l}\text { Malakondaiah et al, } \\
1979\end{array}$ & $\begin{array}{l}\text { Malakondaiah et al, } \\
1979\end{array}$ & $\begin{array}{c}\text { Malakondaiah et al, } \\
1979\end{array}$ \\
\hline Olivine & orthorhombic & $\begin{array}{l}\text { Kohlstedt et al, 1974, } \\
\text { Justice et al, } 1982\end{array}$ & $\begin{array}{l}\text { Kohlstedt et al, 1974, } \\
\text { Justice et al, } 1982\end{array}$ & Frost et al, 1982 \\
\hline
\end{tabular}




\section{a. Harper-Shepard-Dorn jogged screw dislocation theory}

The earliest H-D creep theory has been proposed by Harper and Dorn, 1958 to explain their original $\mathrm{Al}$ results. They assumed that the rate controlling process is the formation and diffusion of vacancies necessary for the jog-controlled motion of the screw dislocation segments. Their final expression for the steady state creep rate had the following form:

$$
\dot{\varepsilon}=\frac{v_{o} \sigma \rho b^{5} N_{a}}{6 R T} \exp \left(-\frac{\Delta H_{s d}+\Delta H_{p}-\Delta H_{j}}{R T}\right)
$$

where $v_{\mathrm{o}}$ is Debye frequency, $\mathrm{N}_{\mathrm{a}}$ is the Avogadro's number, $\mathrm{R}$ the gas constant, and $\Delta \mathrm{H}_{\mathrm{sd}}$, $\Delta \mathrm{H}_{\mathrm{p}}$ and $\Delta \mathrm{H}_{\mathrm{j}}$ are activation energies for the self diffusion, overcoming Peierls force and jog formation, respectively. This equation can be expressed in terms of glide velocity of screw dislocations with jogs, eq. (1.31), by noticing that the mean equilibrium jog spacing was assumed

$$
l_{j}=b \exp \left(\Delta H_{j} / R T\right) \sim 6 b
$$

and self diffusion coefficient equal

$$
D_{s}=v_{o} b^{2} \exp \left(-\frac{\Delta H_{s d}+\Delta H_{p}}{R T}\right)
$$

Incorporating eqs. (1.35) and (1.36) into (1.34) gives

$$
\dot{\varepsilon}=\frac{b}{6} \rho l_{j} \frac{D_{s} \sigma b}{k_{B} T}=\frac{1}{24 \pi} \rho b v_{g j}
$$

where the factor $1 / 24 \pi$ comes from the conversion of jog movement to the area swept by the dislocation. Langdon and Yavari 1982 refined this approach and derived an alternative relation in the form:

$$
\dot{\gamma}=12 \pi \rho l_{j} b\left(\frac{D_{s} G b}{k_{B} T}\right)\left(\frac{\tau}{G}\right)
$$

where $\mathrm{G}$ is shear modulus. Converting shear stresses and strains to their tensile equivalent using relations (Yavari 1982) 


$$
\tau=\frac{\sigma}{2} \quad \text { and } \quad \dot{\gamma}=\frac{3}{2} \dot{\varepsilon}
$$

produces

$$
\dot{\varepsilon}=\frac{12 \cdot 2}{3 \cdot 2} \pi \rho l_{j} b\left(\frac{D_{s} G b}{k_{B} T}\right)\left(\frac{\sigma}{G}\right)=\rho b\left(\frac{4 \pi l_{j} D_{s} \sigma b}{k_{B} T}\right)=\rho b v_{g j}
$$

which means that the above relation is indeed consistent with the Orowan strain rate equation with the dislocation velocity equal to the glide velocity of jogged screw dislocation. Combining eqs. (1.40) and (1.35) gives the following normalized form of the steady-state creep equation

$$
\frac{\dot{\varepsilon} k_{B} T}{24 \pi \Omega D_{s} G \rho}=\frac{\sigma}{G}
$$

which is compared with experimental data in Figure 1. The general trend indicates that

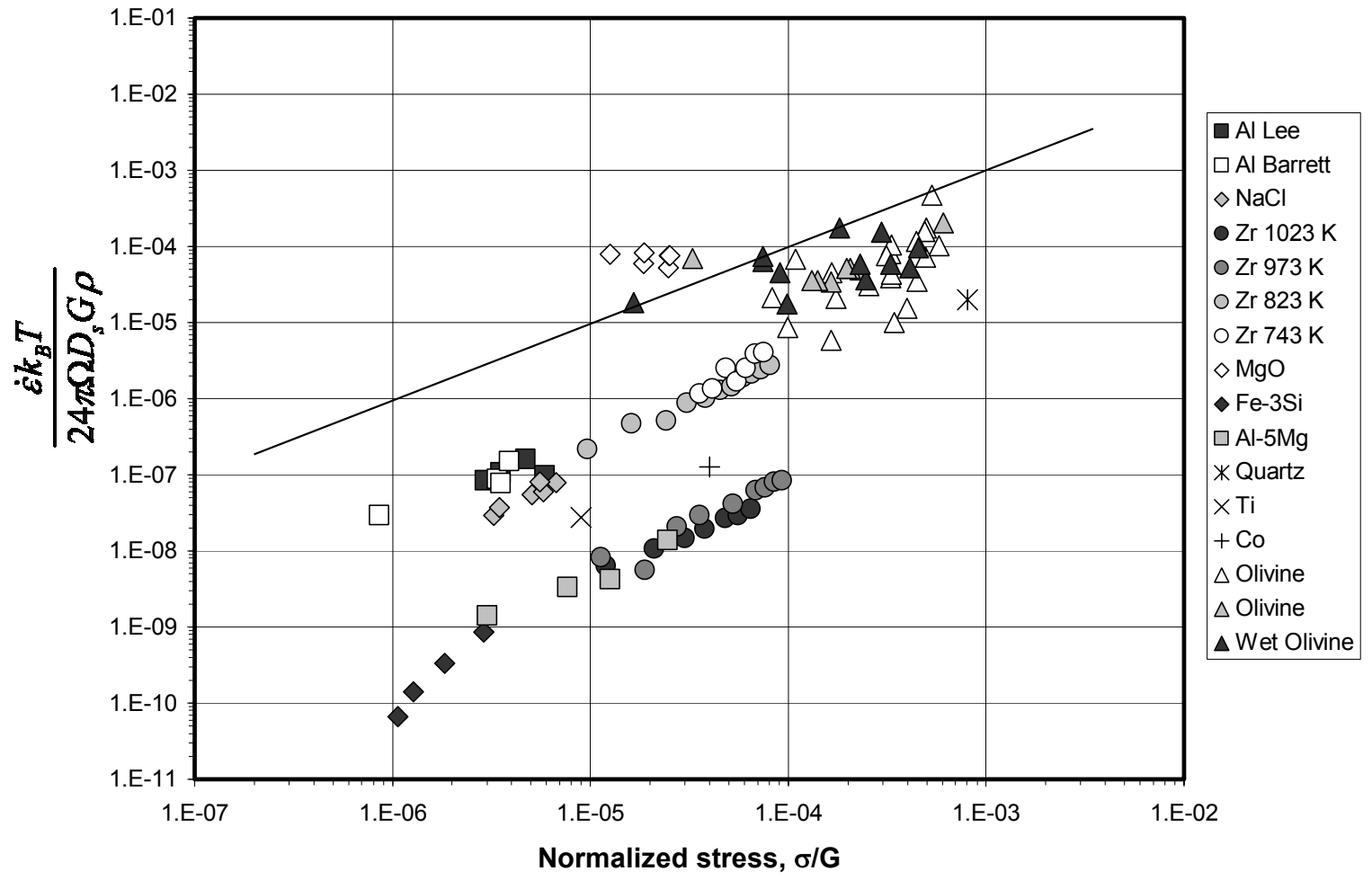

Figure 1. Comparison of the predictions from the jogged screw dislocations glide theory, eq.(1.41), represented by the straight line with the experimental data for H-D creep from data sources listed in Table 1. 
the theory overestimates steady-state creep rates in the lower stress range of the H-D creep regime. The list of advantages and disadvantages of the above model follows.

\section{Advantages:}

- the model predicts linear dependence of creep rate on stress

- predicted strain rates are in good agreement with the original Harper and Dorn experimental data for aluminum

\section{Disadvantages:}

- the assumed screw dislocations are not observed during H-D creep

- the jog spacing obtained from the above relation and strain rate data for Al is less then $b$ which is unrealistically small

- theory does not explain why dislocation density is independent of stress

\section{b. Barrett-Muehleisen-Nix edge dislocation climb theory}

The next attempt to explaining the H-D behavior was due to Barrett et al. 1972. They assumed that the H-D creep results from the diffusion-controlled climb of edge dislocation generated by the fixed number of sources. The steady state dislocation density, $\rho$, was estimated from the condition of the balance between dislocation generation, $\dot{\rho}_{+}$, the annihilation, $\dot{\rho}_{-}$, rates. The former was assumed proportional to the dislocation density generated per one climb event, $\rho_{o}$, divided by the time of climb

$$
\dot{\rho}_{+}=\rho_{o} \frac{v_{c}}{x}
$$

where $v_{c}$ is the climb velocity given by eq. (1.11) and $\mathrm{x}$ is climb distance. $\rho_{o}$ was assumed constant and equal to the as-annaled dislocation density, and climb distance was obtained from the standard stress-dislocation density relation

$$
\sigma \propto G b \sqrt{\rho}=\frac{G b}{x}
$$

which gives

$$
x=\frac{G b}{\sigma}
$$


Substituting expressions for $\mathrm{x}$ and $v_{\mathrm{c}}$ into eq. (1.42) gives dislocation generation rate equal to

$$
\dot{\rho}_{+}=\frac{\rho_{o} D_{s} b \sigma^{2}}{G k_{B} T}
$$

As to the annihilation rate, $\dot{\rho}_{-}$, it was obtained by assuming that dislocation disappear into the subgrain boundaries. Consequently, $\dot{\rho}_{-}$is equal to the dislocation density divided by the time it takes for a dislocation to cross the subgrain

$$
\dot{\rho}_{-}=\frac{\rho v_{g}}{\lambda}
$$

where $v_{g}$ is glide velocity and $\lambda$ is subgrain diameter assumed inversely proportional to stress

$$
\lambda=\frac{\lambda_{o}}{\sigma}=\frac{2 G b \xi}{\sigma}
$$

The first form of the last relation is due to Barrett et al. 1972 and the second to Langdon and Yavari 1982. From experimental data for Al it was determine that $\lambda_{\mathrm{o}}=68.9$ $\mathrm{N} / \mathrm{m}$ while $\xi \sim 10$. Glide velocity was assumed linearly proportional to stress:

$$
v_{g}=v_{o} \sigma
$$

where $v_{o}$ is a temperature dependent constant. Consequently

$$
\dot{\rho}_{-}=\frac{\rho v_{o} \sigma^{2}}{2 G b \xi}
$$

At steady state conditions

$$
\dot{\rho}_{+}=\dot{\rho}_{-} \quad \text { or } \quad \frac{\rho_{o} D_{s} b \sigma^{2}}{G k_{B} T}=\frac{\rho v_{o} \sigma^{2}}{2 G b \xi}
$$

which solved for steady state dislocation density gives:

$$
\rho=\frac{2 \rho_{o} D_{s} b^{2} \xi}{k_{B} T v_{o}}
$$

Substituting result to the expression for strain rate, eq. (1.1) produces

$$
\dot{\varepsilon}=\frac{2 \rho_{o} D_{s} b^{3} \xi \sigma}{k_{B} T}
$$

or in terms of climb velocity $v_{c}$ given by eq. (1.11) as 


$$
\dot{\varepsilon}=2 \xi \rho_{o} b v_{c}
$$

which suggests that the initial dislocation density $\rho_{o}$ controls both steady state dislocation density and the creep rate. Eq. (1.52) can be also written in a standard creep literature form as

$$
\dot{\varepsilon}=2 \rho_{o} b^{2} \xi\left(\frac{D_{s} G b}{k_{B} T}\right)\left(\frac{\sigma}{G}\right)=\frac{\rho_{o} b \lambda_{o}}{G}\left(\frac{D_{s} G b}{k_{B} T}\right)\left(\frac{\sigma}{G}\right)
$$

where the first form is due to Langdon-Yavari 1982 and the second Barrett et al. 1972 (in Langdon-Yavari paper the numerical constant is in error equal to 4/3). The theory predicts then correctly that strain rate is proportional to $\sigma$ and that dislocation density is independent on stress. However, if subgrains were not present, then dislocations would need to annihilate at grain boundaries. For the grain size $d$ the annihilation rate will be then

$$
\dot{\rho}_{-}=\frac{\rho v_{o} \sigma}{d}
$$

hence, following above procedure, the steady state dislocation density becomes:

$$
\rho=\frac{\rho_{o} D_{s} b d \sigma}{G k_{B} T v_{o}}
$$

and, as a result

$$
\dot{\varepsilon}=\rho_{o} b^{2}\left(\frac{D_{s} G b}{k_{B} T}\right)\left(\frac{d}{b}\right)\left(\frac{\sigma}{G}\right)^{2}
$$

The steady-state strain rate is now proportional to the stress to power 2 and dislocation density is linear with stress. Unfortunately predictions from both eqs. (1.54) and (1.57) cannot be compared with the experimental data because of unavailability of data on initial dislocation density, $\rho_{o}$, and grain size, $d$, for materials listed in Table 1 . The main benefits and drawbacks of the above approach are listed below.

\section{Advantages}

- predicted stress exponent equal one

- predicted steady-state dislocation density is independent on stress

- theory has good predictive capabilities

\section{Disadvantages}


- requires presence of the subgrain boundaries.

\section{c. Climb of jogged edge dislocations}

Using derived earlier expression for climb velocity of jogged edge dislocation, $v_{c e j}$, eq. (1.19), the steady-state creep rate is equal to:

$$
\dot{\varepsilon}=\frac{4 \pi b^{3} \rho}{l_{j c}} \frac{D_{s} b G}{k_{B} T} \frac{\sigma}{G}
$$

Using above relation and data for H-D creep on Al obtained by Lee 1985, the estimated jog spacing at $920 \mathrm{~K}$ is in the order of $\sim 5 \mathrm{~b}$. On the other hand, according Langdon et al. 1982 and Ardell et al 1965, the theoretical thermal equilibrium jog spacing is for Al equal to

$$
l_{j c}=\frac{b}{10} \exp \left(\frac{U_{j}}{k_{B} T}\right) \approx 15 b
$$

which was obtained using activation energy for jog formation equal $0.4 \mathrm{eV}$ (Langdon et al. 1982). The experimental jog spacing is then three times smaller then expected. Combining eqs. (1.58) and (1.59) gives the following normalized form of the creep rate equation:

$$
\frac{15 \dot{\varepsilon} k_{B} T}{4 \pi \Omega \rho D_{s} G}=\frac{\sigma}{G}
$$

Predictions from that equation have been compared with experimental data in Figure 2. The predictions of this theory are in surprisingly good agreement with experimental data despite the fact that the theory neglects expected supersaturation effects. Below is the summary of advantages and disadvantages of the above approach:

\section{Advantages:}

- predicted stress exponent equal one

- theory is consistent with the expected presence of edge dislocations

\section{Disadvantages:}

- for $\mathrm{Al}$ at $920 \mathrm{~K}$ the estimated steady state creep rate is three times smaller than expected 


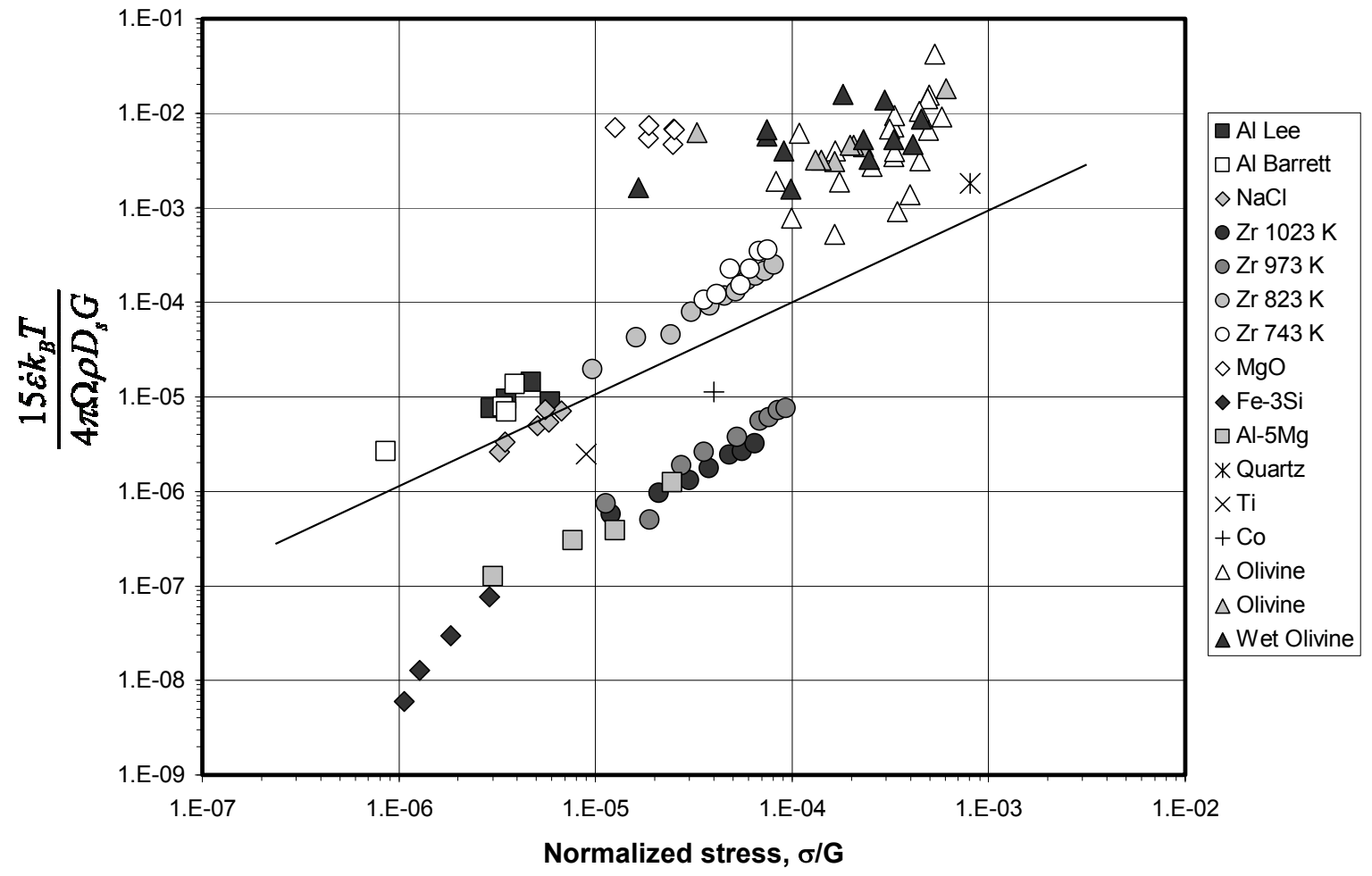

Figure 2. Comparison of the predictions from the jogged edge dislocation climb theory, eq.(1.60), represented by the straight line with the experimental data for H-D creep from data sources listed in Table 1.

- high jog density should result in vacancy supersaturation which is not taken into account.

\section{d. Climb of edge dislocations under supersaturated conditions}

This creep mechanism was originally proposed by Fridel 1964 and subsequently adopted for H-D creep by Mohamed et al. 1975. It is assumed that the jogs on the edge dislocations are saturated with vacancies and as a result the movement of dislocation is

controlled by the diffusion of vacancies to/from the dislocation line. Using strain rate eq. (1.1) with the climb velocity $v_{\text {css }}$ for the supersaturation conditions, eq. (1.28), one obtains:

$$
\dot{\varepsilon}=\frac{2 \pi b^{2} \rho}{\ln \left(\frac{1}{b \rho^{1 / 2}}\right)}\left(\frac{D_{s} b G}{k_{B} T}\right)\left(\frac{\sigma}{G}\right)
$$


The equation gives then correct dependence of strain-rate on the stress to the power of one. The predictions from this approach using normalized version eq. (1.61) in the form

$$
\frac{\dot{\varepsilon} k_{B} T}{2 \pi \Omega D G} \ln \left(\frac{1}{b \rho^{1 / 2}}\right)=\frac{\sigma}{G}
$$

are shown in Figure 3. It is quite evident that the predicted H-D creep rates are in general in good agreement with experimental data.

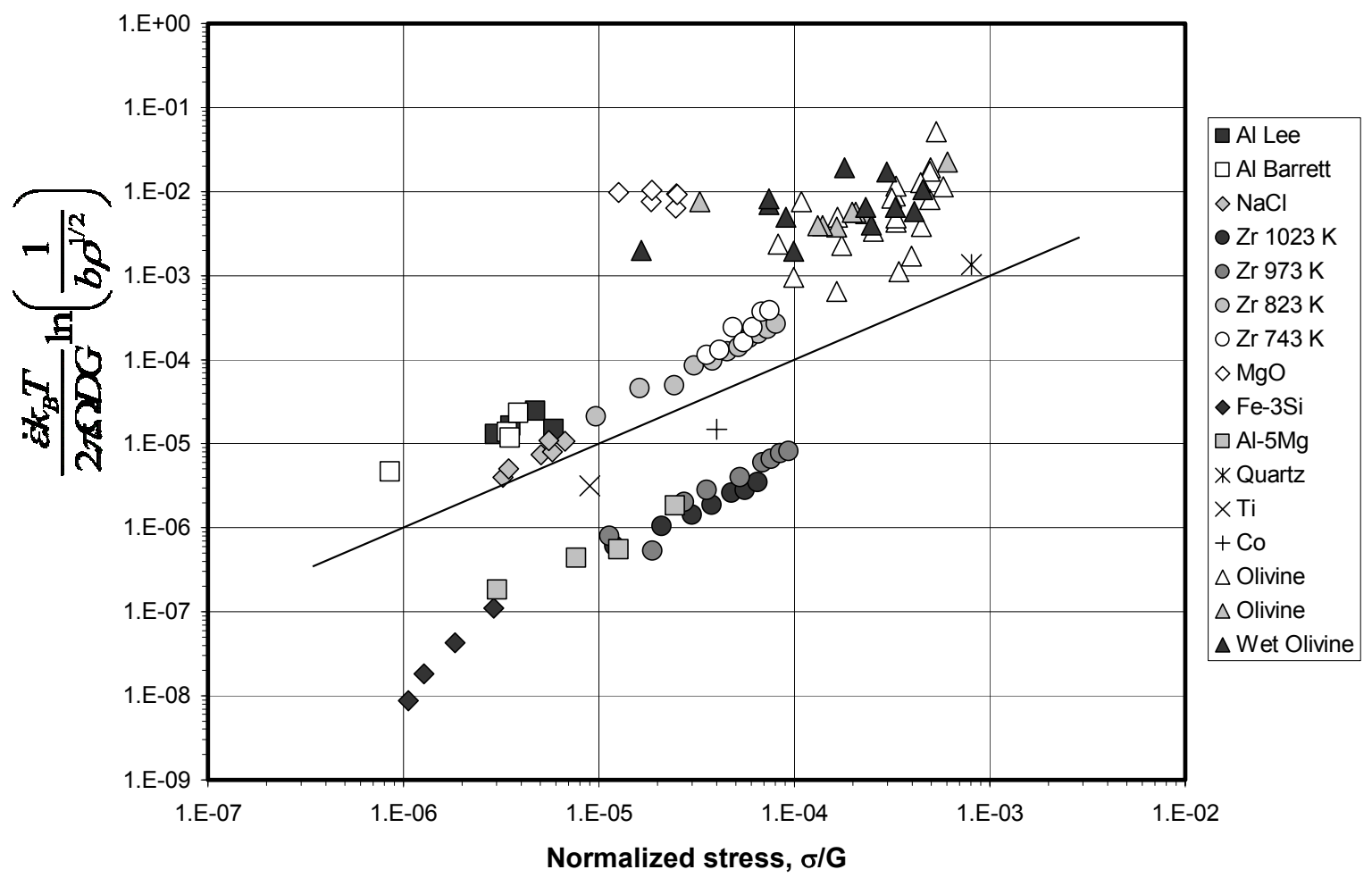

Figure 3. Comparison of the predictions from theory of climb of edge dislocations under supersaturation conditions, eq.(1.62), represented by the straight line with experimental data for H-D creep from sources listed in Table 1.

To validate the main assumption of this approach it is necessary to check if saturation condition indeed exist during H-D creep. According to Langdon et al 1982 and Fridel 1964 the saturation takes place when:

$$
\ln \left(\frac{\Lambda}{b}\right)>2.5
$$

where $\Lambda$ is a characteristic distance for vacancy diffusion equal: 


$$
\Lambda=b \ln \left(\frac{r}{b}\right)
$$

where $\mathrm{r}$ is in this case dislocation spacing assumed equal:

$$
r=\rho^{-1 / 2}
$$

Combining last three relations gives saturation condition in the form:

$$
\ln \left(\frac{1}{b \rho^{1 / 2}}\right)>12.18 \quad \text { or } \quad \rho<2.62 \cdot 10^{-11} b^{-2}
$$

As an example, for $\mathrm{Al}$ at $920 \mathrm{~K}$ this condition gives $\rho<3.2 \cdot 108 \mathrm{~m}^{-2}$. Comparing with the experimental value of dislocation density of about $8 \cdot 107 \mathrm{~m}^{-2}$ the predicted value is four times larger which means that H-D creep indeed takes place under condition of supersaturation.

Langdon and Yavari 1982 also developed a condition for the limiting grain size resulting in the transition from $H-D$ creep under vacancy supersaturation to the diffusion controlled Nabarro-Herring or Coble creep. For the Harper-Dorn they assumed the strain rate given by relation:

$$
\dot{\varepsilon}=28\left(\frac{D_{s} G b}{k_{B} T}\right)\left(\frac{b}{d}\right)^{2}\left(\frac{\sigma}{G}\right)
$$

Equating this strain rate with the one for supersaturation controlled H-D creep, eq. (1.61), and solving for the grain size, $\mathrm{d}$, gives:

$$
d_{N-H}>\sqrt{\frac{14}{\pi \rho} \ln \left(\frac{1}{b \rho^{1 / 2}}\right)}
$$

For Al, using experimental $\rho$ this condition gives the grain size for H-D creep grater than about $850 \mu \mathrm{m}$. Using dislocation density from the supersaturation conditions transforms the last relation to:

$$
d_{N-H}>b e^{e^{2,5}} \sqrt{\frac{14}{\pi} e^{2.5}} \approx 1.439 \cdot 10^{6} b
$$

which gives transitional grain size equal $412 \mu \mathrm{m}$. For Coble creep the strain-rate equation given by Langdon and Yavari 1982 has the form:

$$
\dot{\varepsilon}=\frac{1.03 \times 10^{2}}{\pi}\left(\frac{\delta}{b}\right)\left(\frac{D_{g b} G b}{k_{B} T}\right)\left(\frac{b}{d}\right)^{3}\left(\frac{\sigma}{G}\right)
$$


where $\delta$ and $D_{g b}$ are grain boundary width and diffusion, respectively. The transition grain size is in this case equal to

$$
d_{C}>\left\{\frac{1.03 \times 10^{2}}{2 \pi^{2}} \frac{D_{g b}}{D} \frac{\delta}{\rho} \ln \left(\frac{1}{b \rho^{1 / 2}}\right)\right\}^{1 / 3} \cong\left\{5.23 \frac{D_{g b}}{D} \frac{\delta}{\rho} \ln \left(\frac{1}{b \rho^{1 / 2}}\right)\right\}^{1 / 3}
$$

Replacing dislocation density with theoretical saturation value gives:

$$
d_{C}>\left\{\frac{1.03 \times 10^{2}}{2 \pi^{2}} \frac{\delta D_{g b}}{D} b^{2} e^{2.5+2 e^{2.5}}\right\}^{1 / 3}=1.344 \times 10^{4}\left(\frac{\delta b^{2} D_{g b}}{D}\right)^{1 / 3}
$$

The H-D theory of edge dislocation climb under superasaturation conditions has than the following advantages and shortcomings:

\section{Advantages:}

- predicts linear relation with stress to the power of one

- creep rate is independent on grain size

- consistent with primary creep behavior

- steady state dislocation density does not depend on stress

- does not preclude presence of subgrains

- consistent with observations of the dominance of edge dislocations

- valid both for pure metals and solid solutions and is independent of solute concentration

\section{Disadvantages:}

- predicted creep rate is 4 to 5 times smaller than expected rate for Al.

- predicted steady state dislocation density at saturation conditions is for Al for times larger then experimental

- the model does not have provision for dislocation multiplication, hence it cannot account for large strains observed during H-D creep (Mohamed 1991).

\section{e. Internal stress theory}

The internal stress unified approach to the creep behavior has been proposed by $\mathrm{Wu}$ and Sherby 1984 with the elements of that approach introduced earlier by Barrett 1967. The H-D creep model is a special case of a general Wu and Sherby creep model 
which has been subsequently refined by Ruano at al. 1988. The starting relation is in this approach the Garafano, 1963 creep equation

$$
\varepsilon=\frac{K}{\alpha^{n}} \frac{D_{e f f}}{b^{2}}\left(\sinh \alpha \frac{\sigma}{E}\right)^{n}=\frac{K}{\alpha^{n}} \frac{D_{e f f}}{b^{2}}\left(\frac{1}{2} \exp \left(\alpha \frac{\sigma}{E}\right)-\frac{1}{2} \exp \left(-\alpha \frac{\sigma}{E}\right)\right)^{n}
$$

where $K, \alpha$ and $n$ are material constants with $n \sim 5, D_{\text {eff }}$ is effective diffusion coefficient and $\alpha$ is on the order of $\sim E / \sigma$ at the "start of the power law breakdown". The model assumes further that:

- above relation correctly describes diffusion controlled dislocation creep,

- internal stress, $\sigma_{i}$, generated by the dislocations within the subgrains adds to the external stress in driving the motion of half of the dislocations and opposes the motion of the other half

- plastic flow contributions from each half of the dislocations are independent.

As a result the strain rate equation, for tensile deformation, can be written as

$$
\dot{\varepsilon}=\frac{1}{2} \frac{K}{\alpha^{n}} \frac{D_{e f f}}{b^{2}}\left[\left(\sinh \left(\alpha \frac{\sigma+\sigma_{i}}{E}\right)\right)^{n}+\frac{\left|\sigma-\sigma_{i}\right|}{\sigma-\sigma_{i}}\left(\sinh \left(\alpha \frac{\left|\sigma-\sigma_{i}\right|}{E}\right)\right)^{n}\right]
$$

where the coefficient in the second term in the brackets is introduced to allow for negative contribution to creep when external stress is less then $\sigma_{i}$ (expression valid for both tension and compression is also given in the original paper). Since the argument of the sinh is small, one can write:

$$
\left\{\sinh \left(\alpha \frac{\sigma \pm \sigma_{i}}{E}\right)\right\}^{n} \approx\left(\frac{\alpha}{E}\right)^{n}\left(\sigma \pm \sigma_{i}\right)^{n}
$$

which substituted to strain rate equation gives:

$$
\dot{\varepsilon}=\frac{1}{2} A_{P L} \frac{D_{e f f}}{b^{2}}\left\{\left(\frac{\sigma+\sigma_{i}}{E}\right)^{n}+\frac{\mid \sigma-\sigma_{i}}{\sigma-\sigma_{i}}\left|\frac{\sigma-\sigma_{i}}{E}\right|^{n}\right\}
$$

where $A_{P L}=K$ is a power law material constant. In the $H-D$ regime the acting stress, $\sigma$, is much less than the internal stress $\sigma_{\mathrm{i}}$ and we have:

$$
\dot{\varepsilon}=\frac{1}{2} A_{P L} \frac{D_{e f f}}{b^{2}}\left(\frac{\sigma_{i}}{E}\right)^{n}\left\{\left(1+\frac{\sigma}{\sigma_{i}}\right)^{n}-\left(1-\frac{\sigma}{\sigma_{i}}\right)^{n}\right\} \approx \frac{1}{2} A_{P L} \frac{D_{e f f}}{b^{2}}\left(\frac{\sigma_{i}}{E}\right)^{n}\left\{\left(1+n \frac{\sigma}{\sigma_{i}}\right)-\left(1-n \frac{\sigma}{\sigma_{i}}\right)\right\}
$$

which reduces to (Wu 1984): 


$$
\dot{\varepsilon}=A_{P L} n \frac{D_{e f f}}{b^{2}}\left(\frac{\sigma_{i}}{E}\right)^{n-1}\left(\frac{\sigma}{E}\right)
$$

Assuming $\mathrm{n}=5$, replacing Young modulus E with $2 \mathrm{G}(1+v)$ and using Taylor relation in the form (Ruano 1988)

$$
\frac{\sigma_{i}}{E}=0.4 b \sqrt{\rho}
$$

leads to the following expression for the strain rate:

$$
\dot{\varepsilon}=0.128 A_{P L} \frac{D_{e f f}(b \rho)^{2}}{2(1+v)}\left(\frac{\sigma}{G}\right)
$$

The Taylor relation defines than in this case the transition stress to the H-D creep regime. Using dislocation density data for H-D creep of $\mathrm{Al}$ at $920 \mathrm{~K}$ the internal stress estimated from Taylor formula is about $0.05 \mathrm{MPa}$ which is about half of the experimentally observed H-D transition stress. Eq. (1.80) can be written in a normalized form as

$$
\frac{\dot{\varepsilon}(1-v)}{0.64 A_{P L}(b \rho)^{2} D_{e f f}}=\frac{\sigma}{G}
$$

The H-D data from the sources given in Table I are plotted according to that relation in Figure 4.

$\mathrm{Wu}$ and Sherby 1984 also derived an alternative form of the H-D strain rate equation which incorporates effects of the subgrain size. Starting from relation

$$
\dot{\varepsilon}=\frac{K}{\alpha^{n}} \frac{D_{e f f}}{b^{2}}\left(\sinh \alpha \frac{\sigma}{E}\right)^{n} \approx K \frac{D_{e f f}}{b^{2}}\left(\frac{\sigma}{E}\right)^{n}
$$

they additionally assumed that the strain rate in power law regime is proportional to the subgrain size to the power of 3 . To introduce subgrain size, $\lambda$, to the strain rate equation they use power law relation between $\lambda$ and the stress in the form:

$$
\lambda=A b \frac{E}{\sigma} \quad \text { or } \quad 1=\frac{\lambda}{A b} \frac{\sigma}{E}
$$

where for metals $A \sim 4$. Taking last expression to the third power and substituting to the expression for strain rate gives:

$$
\dot{\varepsilon}=\frac{K}{A^{3}} \frac{D_{e f f}}{b^{2}}\left(\frac{\lambda}{b}\right)^{3}\left(\frac{\sigma}{E}\right)^{n+3}=\frac{K}{A^{3}} \frac{D_{e f f}}{b^{2}}\left(\frac{\lambda}{b}\right)^{3}\left(\frac{\sigma}{E}\right)^{8}
$$




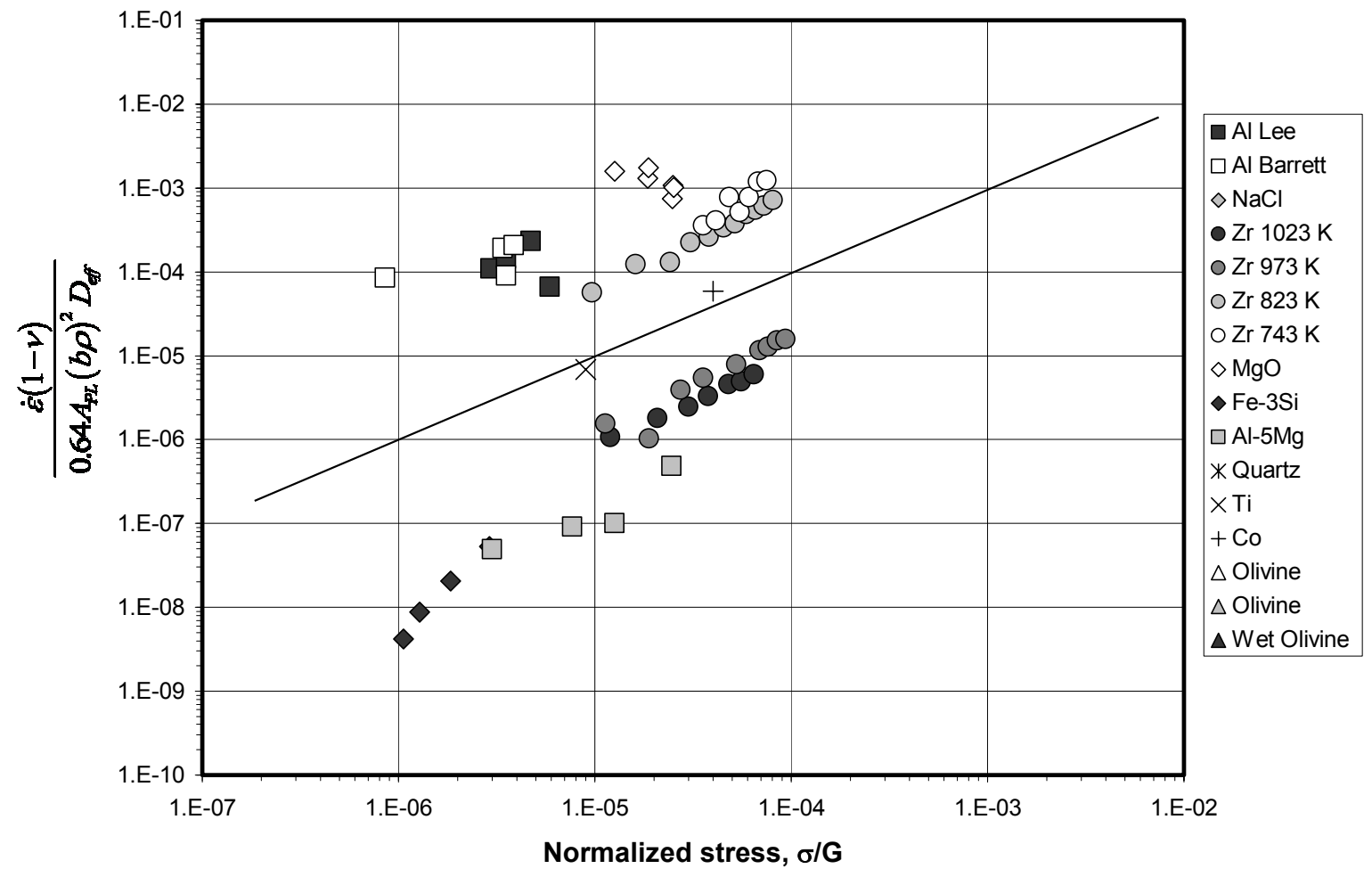

Figure 4. Comparison of the predictions from internal stress theory, eq. (1.81), represented by the straight line with experimental data for H-D creep from sources listed in Table 1. The values of $\mathrm{A}_{\mathrm{PL}}$ are from Ruano et al, 1988.

which is valid in the power law region. Introducing internal stress the same way as before transforms above relation to:

$$
\dot{\varepsilon}=\frac{1}{2} \frac{K}{A^{3}} \frac{D_{e f f}}{b^{2}}\left(\frac{\lambda}{b}\right)^{3}\left[\left(\frac{\sigma+\sigma_{i}}{E}\right)^{8}+\frac{\left|\sigma-\sigma_{i}\right|}{\sigma-\sigma_{i}}\left(\frac{\sigma-\sigma_{i}}{E}\right)^{8}\right]
$$

and in the H-D regime of small $\sigma<\sigma_{i}$ to

$$
\dot{\varepsilon}=\frac{8 K}{A^{3}} \frac{D_{e f f}}{b^{2}}\left(\frac{\lambda}{b}\right)^{3}\left(\frac{\sigma_{i}}{E}\right)^{7}\left(\frac{\sigma}{E}\right)
$$

The above relation predicts then that the strain rate increases with subgrain size and dislocation density through increase of $\sigma_{i}$. The strongest and weakest points of the internal stress approach to the H-D creep are summarized below.

\section{Advantages:}

- creep rate linear with stress 
- good predictive capabilities

- capable of describing power-law and H-D creep rates with one equation

- suggests that high temperature creep is of the H-D rather than Nabarro-Herring type

- suggested annihilation mechanism is climb hence multiplication is by operation of F-R sources.

- $\sigma_{i}$ obtained from fitting theory to data correlates well with dislocation density (fig 4 Ruano 1988)

- suggests that if dislocation density decreases with increasing temperature, this change would result in the apparent decrease of the activation energy for creep beyond that of lattice diffusion as observed by Novotny et al 1985 in Zr.

\section{Disadvantages:}

- theory does not provide explanation of how the steady state develops, or for multiplication and annihilation mechanisms

- theory is inconsistent with the primary creep behavior - large initial dislocation density suggests increase rather observed decrease of the steady-state creep rate (Ardell 1997)

- diffusion of vacancies at high temperatures would relieve all internal stresses (Ardell 1997)

- theory suggests that H-D creep is an extension of power-law creep although these two creep regimes are controlled by different processes, e.g. dislocation density is independent on stress in H-D creep while it is proportional to the square of stress in the power-law creep region (Nabarro 1989, Mohamed et al. 1991).

- theory suggests that there are fluxes of dislocation similar to those of vacancies. This is incorrect because dislocations are not in thermal equilibrium like vacancies (Nabarro 1989)

- the dislocation travel distance corresponding to the H-D transient strain of $10^{-4}$ is about $6 \mathrm{~mm}$ while for the total strain of about $10^{-1}$ it would be about $6 \mathrm{~m}$. These distances are too large (Nabarro 1989). 
- if the H-D creep is an extension of the power-law creep it should have both $\sigma_{\mathrm{i}}$ and $\rho^{1 / 2}$ proportional to stress. In H-D creep region $\rho$ is independent of stress (Nabarro 1989).

\section{f. Ness H-D creep theory}

In his review of the work hardening models of fcc metals Ness questioned the assumption of the H-D creep models that dislocation density is independent on stress (Ness 1998). He showed that:

- his results on dislocation density on Al quoted by Mohamed eat al. 1973 and Yavari et al. 1982 do not show, as claimed, that dislocation density is independent of stress but rather that $\rho \propto \sigma^{1.3}$.

- the Yavari et al 1982 conclusion that during H-D creep dislocation have predominantly edge character is based on the measurements on the foils that had 5 to 6 orders of magnitude higher dislocation density that the one expected for H-D creep

He also proposed a H-D creep theory based on the balance of the dislocation generation/storage and annihilation rates similar in principle to that of Barrett et al. 1972. The key assumptions of the Ness theory are as follows:

i. The H-D creep is similar to the Stage I deformation in single crystals because steady state is reached at strains on the order of $1 \%$ hence under single slip conditions.

ii. The density of dislocation sources is independent of stress: each source generates $N$ loops traveling average distance $\mathrm{L}$ and forming slip lines separated by distance $y$. In Stage I $L$ and y are independent on stress which gives source and generated dislocation densities equal to:

$$
\begin{gathered}
\text { source density }=\left[y L^{2}\right]^{-1} \\
\rho=\text { loop length } \times \text { source density } \approx 2 \pi L N \times \frac{1}{y L^{2}}=\frac{2 \pi N}{y L}
\end{gathered}
$$

According to Ness it is expected that for $\mathrm{Al} \mathrm{L} \sim 10^{-2}-10^{-3} \mathrm{~m}$ and $\mathrm{y} \sim 10^{-4}-10^{-5} \mathrm{~m}$. 
iii. The dislocation storage/generation rate is estimated as the ratio of the generated dislocation density to the resulting shear strain. Since the generated strain is

$$
\gamma=\frac{N b(\text { Swept Area })}{\text { Volume }} \approx \frac{N b L^{2}}{y L^{2}}=\frac{N b}{y}
$$

then the dislocation storage/generation rate is equal to

$$
\frac{d \rho^{+}}{d \gamma}=\frac{2 \pi N}{y L} \frac{y}{N b}=\frac{2 \pi}{b L}
$$

iv. Following Seeger 1961 the "back" stress on the source from "superdislocation" of strength $N b$ is estimated similar to Stage I flow stress as:

$$
\tau_{s}=\frac{\left(y^{3} L\right)^{1 / 4}}{4 \pi^{2}} G b \rho
$$

v. The dislocation recovery/annihilation rate is assumed to be controlled by the collapse of edge dipoles which is proportional to dislocation density and inversely proportional to the strain per one collapse event

$$
\frac{d \rho^{-}}{d \gamma}=-\rho \frac{v_{g}}{\dot{\gamma}}
$$

where $\mathrm{v}_{\mathrm{g}}$ is dipole collapse frequency.

From the condition that at steady state the sum of generation and annihilation rates is zero Ness obtained the steady state dislocation density equal to

$$
\rho=\frac{\pi y^{2}}{\Omega L B_{\rho}}\left(\frac{\dot{\gamma}}{v_{o}}\right)\left(\frac{k_{B} T}{G \Omega}\right) \exp \left(\frac{Q_{s}}{k_{B} T}\right)
$$

where $B_{\rho}$ is material constant and $Q_{s}$ activation energy for self diffusion. Eliminating dislocation density from eqs. (1.91) and (1.93) gives the following expression for the steady-state strain rate (Ness 1998):

$$
\dot{\varepsilon}=\frac{b^{2} L}{y^{2}\left(y^{3} L\right)^{1 / 4}} v_{o}\left(\frac{\sigma \Omega}{k_{B} T}\right) \exp \left(-\frac{Q_{s}}{k_{B} T}\right)
$$

Both eqs. (1.93) and (1.94) contain tacit assumption that annihilation/recovery takes place by climb of edge dislocations under supersaturation conditions. Lack of information about the values of $\mathrm{L}$ and $\mathrm{y}$ for materials other than aluminum precludes comparison of 
the predictions from this theory with the experimental data. The most important aspects of the theory are summarized below:

\section{Advantages}

- predicted creep rate stress exponent is equal one

- predicted steady-state dislocation density is independent on stress

- theory has good predictive capabilities

\section{Disadvantages}

- requires presence of active dislocation sources which is inconsistent with the low value of acting external stress

- assumes operation of a single rather than multiple slip mechanisms which are expected to be active at temperatures close to the melting point.

- does not explain transient creep behavior.

\section{g. Peirels stress theories}

The Peirels stresses approach has been suggested by Nabarro 1989 and subsequently refined by Wang et al. 1994, Owns et al. 1996 and Wang 1995, 1996. It is assumed that (1) the H-D creep is controlled by the climb of the edge dislocations under supersaturated vacancy conditions and (2) that transition to H-D creep takes place when acting stress is smaller than either Peierls or internal stress. Under those conditions the steady-state creep rate is given by eq. (1.61). It is further assumed that H-D steady-state dislocation density is related to the Peierls stress, $\tau_{\mathrm{p}}$, through Taylor relation in form

$$
\rho^{1 / 2}=\frac{\tau_{p}}{G b}
$$

Combining eqs. (1.61) and (1.95) and assuming $\Omega=0.7 \mathrm{~b}^{3}$ gives (Owen et al. 1996)

$$
\dot{\varepsilon}=A_{H D} \frac{D_{s} G b}{k_{B} T} \frac{\sigma}{G}
$$

where

$$
A_{H D}=-\frac{1.4 \pi\left(\tau_{p} / G\right)^{2}}{\ln \left(\tau_{p} / G\right)} \approx 0.5\left(\frac{\tau_{p}}{G}\right)^{2}
$$

The values of $A_{H D}$ estimated using above relation with Peierls stress equal to 


$$
\tau_{p}=\frac{2 G}{1-v} \exp \left(-\frac{2 \pi L}{b} \frac{3-2 v}{4(1-v)}\right)
$$

where $\mathrm{L}$ is distance between atomic planes, have been compiled in Wang 1995. In the further refinement of the theory Wang 1996 used slightly different expression for Peirels stress

$$
\tau_{p}=\frac{G}{1-v} \exp \left(-\frac{2 \pi}{1-v} \frac{L}{b}\right)
$$

which, with few additional modifications, led to the following expression for $\mathrm{A}_{\mathrm{HD}}$

$$
A_{H D}=1.4\left(\frac{\tau_{p}}{G}\right)^{2}
$$

The values of $A_{H D}$ for various materials estimated using that relation are given in Wang 1996a. The normalized form of the resulting steady-state creep eq. (1.96) is then

$$
\frac{\dot{\varepsilon} k_{B} T}{A_{H D} D_{s} G b}=\frac{\sigma}{G}
$$

This relation is compared with experimental data in Figure 5 below. The Peirels stresses theory of H-D creep has the following advantages and deficiencies:

\section{Advantages:}

- predicted creep rate stress exponent is equal one

- good agreement between predicted and experimental creep rates

- excellent predictive capabilities with respect to the H-D transition stress

\section{Disadvantages:}

- theory is inconsistent with the primary creep behavior

- as pointed out by Nabarro (1997 and 1997a), Wang's 1996 results should be "vitiated" because of the error in his Peirels stress equation

- there is no adequate explanation why H-D stresses smaller than Peierls stress can cause dislocation multiplication. 


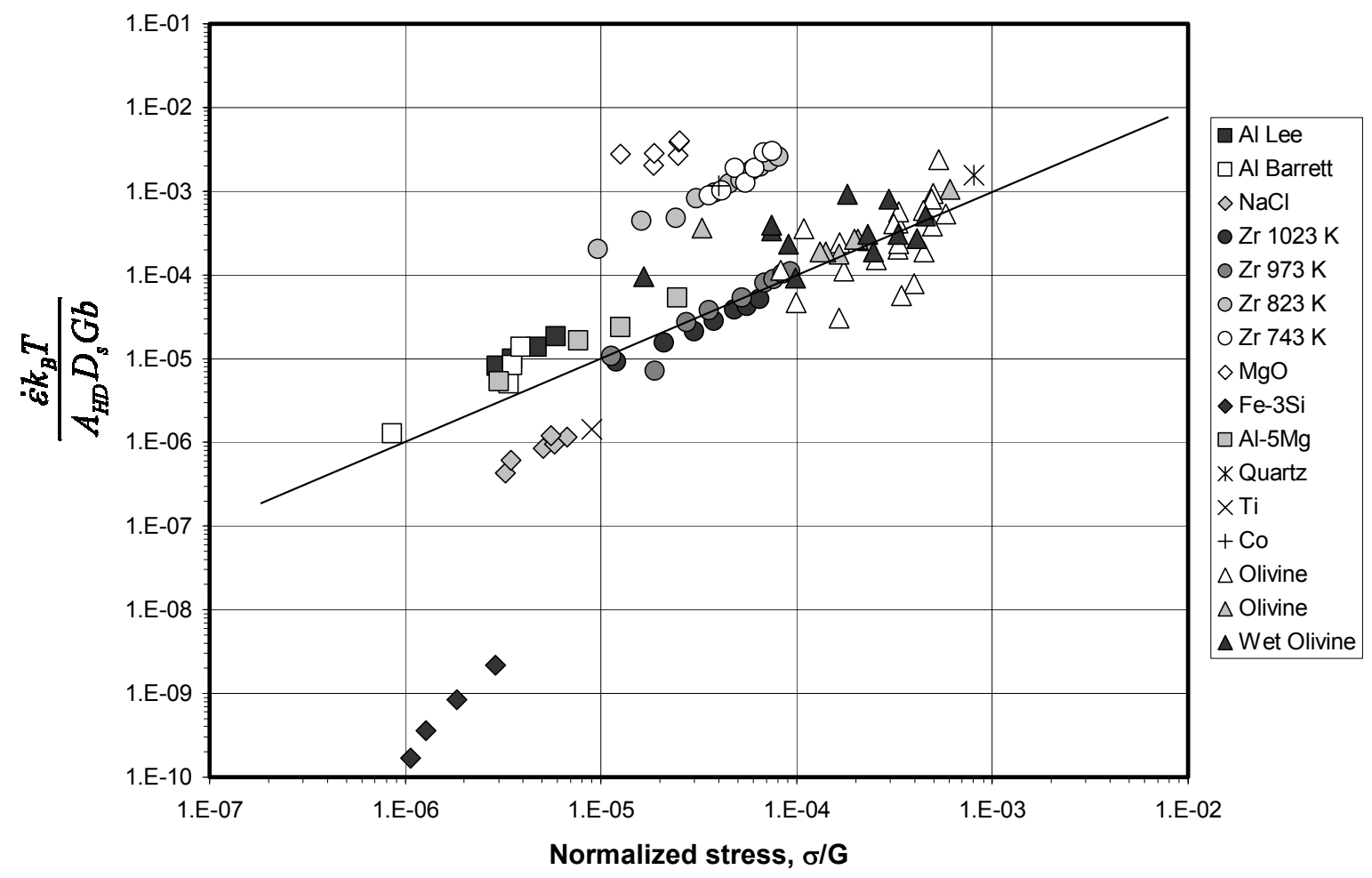

Figure 5. Comparison of the predictions of the Peirels theory, eq. (1.101), represented by the straight line with experimental data for H-D creep from sources listed in Table 1. The values of $A_{H D}$ are from Wang 1996a.

\section{h. Size effect theories}

There are two theories that propose to explain H-D behavior based on the sample size effect. The first one of them has been proposed by Raj 1985, 1987 and Raraman and Raj 1985. It assumes that the H-D creep is a bulk mechanisms controlled by the operation of surface Frank-Read or Bardeen-Herring sources. In the case of Frank-Read sources it is shown that the stress to activate surface source is half of that required to operate a source inside the sample. The Bardeen-Herring sources operate under the action of climb forces triggered by the flow of vacancies from the surface to the dislocation line. The faster of the two mechanisms is the acting one and the generated dislocations propagate by unspecified climb mechanism. The authors determined that these mechanisms control H-D creep when the ratio of specimen thickness or diameter, t, to the grain size, $d$, is 


$$
\mathrm{t} / \mathrm{d}>4
$$

which leads to dependence of the H-D creep rate on the grain size

$$
\dot{\varepsilon} \propto d^{-p}
$$

where $1<p<2$. Based on the experimental data the authors also find that the H-D creep transition stress can be described by relation

$$
\left(\frac{\sigma}{G}\right)_{\text {trans }}=7.3 \times 10^{-6}\left(\frac{t}{d}\right)^{0.8}
$$

The main characteristics of Raj approach are summarized below:

\section{Advantages:}

- good predictive capabilities with respect to the H-D transition stress

- good agreement between predicted and experimental creep rates

\section{Disadvantages:}

- theory is inconsistent with the primary creep behavior

- the obtained transition stress and creep rate dependence on grain size is strongly influence by one uncertain data point (Ardell 1986).

The second size effect theory has been proposed by Ness and coworkers (Ness 2002). This theory is en extension of the described earlier Ness H-D approach based on his unified microstructure evolution model. The unified model has been developed in five papers (Ness 1998, Ness et al. 2001, 2002, 2002a, 2002b), 97 pages total, with numerous adjustable parameters. The size effect H-D theory is a special case of that general approach and it assumes that the dislocation generation rate is described by relation

$$
\dot{\rho}^{+}=S \frac{2}{b L} \dot{\gamma}
$$

where $\mathrm{L}$ is an average distance traveled by mobile dislocation before it is intercepted by the network and equal to

$$
L=\frac{C}{\sqrt{\rho}}
$$

with numerical constant $C>1 . \mathrm{S}$ is the size effect parameter that corrects dislocation generation and recovery rates for the effect of $L$ greater than specimen diameter it is described by an arbitrary chosen function 


$$
S=\frac{R^{2}}{\left((R+L)^{2}-R^{2}\right) \lambda+R^{2}}
$$

where $\mathrm{R}$ is specimen radius an $\lambda$ is a parameter of the order of one. The dislocation annihilation/recovery rate is given by relation:

$$
\dot{\rho}^{-}=-2 S^{3 / 2} \rho v_{D} b^{2} B_{\rho} c_{j}\left(\frac{F b^{2}}{k T}\right) \exp \left(\frac{U_{S D}}{k T}\right)-B_{F N} \rho^{2}\left(\frac{G b^{3}}{k T}\right) D_{S D}
$$

where the first term of the relation is due to dynamic and the second due to static recovery. $B_{r}$ and $B_{F N}$ are parameters, $v_{D}$ is Debye frequency, $c_{j}$ is concentration of jogs controlling dislocation climb rate, $U_{S D}$ and $D_{S D}$ are self-diffusion activation energy and diffusion coefficient, respectively, and $\mathrm{F}$ is driving force generated by dislocation curvatures and estimated to be equal

$$
F \approx 100 \sigma b
$$

Examples of the values of all parameters for Al can be found in Ness et al 2002. From the condition that at steady-state dislocation generation rate, eq.(1.105), is equal to annihilation rate, eq.(1.108), one can obtain steady state dislocation density which substituted to the Orowan eq. (1.1) gives (Ness et al 2002)

$$
\dot{\gamma}=\frac{C}{2 \alpha^{3} b^{2}}\left(\frac{G b^{3}}{k T}\right)\left[\frac{B_{F N}}{S}\left(\frac{\tau}{G}\right)^{3} D_{S D}+\sqrt{S} \frac{13.6 b^{2}}{\alpha} \xi^{2} B_{\rho}\left(\frac{\tau}{G}\right)^{4} v_{D} \exp \left(-\frac{U_{S D}}{k T}\right)\right]
$$

where $\xi$ is an additional adjustable parameter in addition to $B_{F N}$ and $B_{\rho}$. The first part in the brackets describes contribution of static and the second that of dynamic recovery. At $\mathrm{H}-\mathrm{D}$ regime $\mathrm{L}>>\mathrm{R}$ hence according to eq. (1.107) parameter $\mathrm{S}$ becomes small which means that the effect of dynamic recovery becomes minimal and steady-state creep rate is controlled by static recovery - this is the specimen size effect. Eq. (1.110) can be then written as

$$
\dot{\gamma}=\frac{C^{3}}{2 \alpha}\left(\frac{G b^{3}}{k T}\right) \frac{B_{F N}}{R^{2}}\left(\frac{\tau}{G}\right) D_{S D}
$$

In H-D regime the strain rate becomes then proportional to the stress to the power of one. The advantages and disadvantages of this approach are listed below:

Advantages:

- steady-state creep rate is proportional to stress to the power of one 
- predicts transition from the H-D to power law creep with increasing stress

- takes into account both static and dynamic recovery

\section{Disadvantages:}

- the theory does not predict that H-D creep dislocation density is independent on stress

- in the transition to the power law creep the predicted stress exponent is 4 rather than expected 5.

- the expression for pivotal parameter S lacks adequate justification.

- it is unclear how under H-D stress regime mobile dislocation can move distances much greater than average dislocation spacing.

- contrary to the experimental observations the theory predicts that steady-state creep depends on the grain size

Both size effect theories contain parameters which are known only for few special cases which precludes comparison of the predictions from those theories with experimental data.

\section{i. Weertman and Blacic theory}

Weertman and Blacic 1984 developed a theory based on the assumption that H-D creep steady-state dislocation density is controlled by the temperature fluctuations during creep test. The theory assumes that the creep rate is given by Orowan relation (1.1) with dislocation climb velocity given by eq. (1.11) written as

$$
v_{c}=\frac{\sigma}{G} \frac{G \Omega}{k_{B} T} \frac{D_{s}}{b}
$$

where $\sigma$ is applied stress. Dislocation density is on the other hand given by Taylor formula

$$
\rho=\beta\left(\frac{\sigma_{c}}{G b}\right)^{2}
$$

where $\sigma_{c}$ is cyclic climbing stress. Substituting last two eqs. to Orowan relation gives steady-state creep rate equal to

$$
\dot{\varepsilon}=\alpha \beta\left(\frac{\sigma_{c}}{G b}\right)^{2} \frac{\sigma}{G} \frac{G \Omega}{k_{B} T} D_{s}
$$


This means that dislocation density is controlled by the cyclic climbing stress, $\sigma_{c}$, which is responsible for dislocation multiplication, while average creep rate is proportional to external stress $\sigma$. The thermal climbing stress comes about from the ever-present temperature fluctuations which cause local equilibrium of concentration vacancies to lag behind its equilibrium value. The maximum value of theis stress for a temperature change by $\delta \mathrm{T}$ can be obtained from eq. (1.3) and it is equal to:

$$
\sigma_{c \max }=\frac{Q_{f} \delta T}{T \Omega}
$$

where $Q_{f}$ is activation energy for the formation of a vacancy. For $\mathrm{Al}$ near melting temperature this stress is estimated by authors to be on the order of 3 to $6 \mathrm{MPa}$, which is much more than a typical H-D creep regime stress of less than $0.1 \mathrm{MPa}$. The presence of stress $\sigma_{c}$ does not guarantee dislocation multiplication. For that to happen the temperature fluctuation period needs to be long enough for dislocation to climb a distance equal to the dislocation spacing. However, is this period is too long, the local vacancy concentration reaches equilibrium and climbing stress reduces to zero. There is then an optimum range of fluctuation periods, $t_{f}$, for which $\sigma_{c} \neq 0$. According to Weertman and Blacic 1984 this range is

$$
\frac{\beta^{1 / 2} k_{B}^{3} T^{5}}{G \Omega D_{S D}}\left(\frac{b}{c_{o} Q_{f} \delta T}\right)^{2}<t_{f}<\frac{G^{2} b^{2} c_{o} Q_{f} \delta T}{\sigma^{3} T \Omega D_{S D} \beta}
$$

As an example, for Al near melting point $40 \mathrm{~s}<\mathrm{t}_{\mathrm{f}}<10^{4} \mathrm{~s}$. The steady-state dislocation density and climbing stress amplitude for those fluctuation periods are given by relations

$$
\rho=\beta\left(\frac{c_{o} Q_{f} \delta T}{G b T \Omega D t \beta}\right)^{2 / 3}
$$

and

$$
\sigma_{c}=\left(\frac{c_{o} Q_{f} \delta T}{T \Omega D t \beta}\right)^{1 / 3}(b G)^{2 / 3}
$$

where $t$ is temperature fluctuation period. For H-D creep of AL with $\delta \mathrm{T}=1 \mathrm{~K}$ and fluctuation periods from 5 to $50 \mathrm{~min}$ the estimated stedy-state dislocation density is $2.3 \times$ $10^{8}$ to $5 \times 10^{7} \mathrm{~m}^{-2}$ which is in very good agreement with the experimental values. The corresponding values of climbing stress amplitude, $\sigma_{c}$, are 0.075 and $0.035 \mathrm{MPa}$ which 
are of the same magnitude as the acting stress. The key features of the theory are summarized below:

\section{Advantages:}

- steady-state creep rate is proportional to stress to the power of one

- predicts steady-state dislocation density independent of stress

- allows for estimates of the effects of temperature cyclic during creep test

\section{Disadvantages:}

- suggests that H-D creep is an artifact of low-amplitude temperature cycling which is not supported by experimental data (Ardell 1986).

- Nabarro pointed out that the estimated activation energy of the proposed mechanism is only 0.69 of the observed activation energy of the H-D creep.

- the theory can not be verified because of the lack of information on temperature fluctuations during H-D creep tests.

\section{j. Intermediate temperature H-D creep theory}

Although H-D creep has been associated with temperatures close to melting point, it has been also observed at homologous temperatures in the 0.4 to 0.6 range in $\alpha$-titanium (Malakondaiah et al, 1982), $\beta$-cobalt (Malakondaiah et al, 1979), $\alpha$-iron (Novotny et al, 1983 ) and $\alpha$-zirconium(Novotny et al, 1984). Novotny et al 1985 determined that at intermediate temperature H-D creep is controlled by glide of screw dislocations with jogs driven by core diffusion. Using slightly modified eq. (1.31) they proposed the following expression to describe steady-creep rate in $\alpha-\mathrm{Zr}$ :

$$
\frac{\dot{\varepsilon}}{D_{c}}=K \rho_{s} l_{j} b \frac{G b}{k T}\left(\frac{\sigma}{G}-\frac{\sigma_{o}}{G}\right)
$$

where $D_{c}$ is dislocation core diffusion coefficient, $K$ is a numerical constant, $\rho_{s}$ is density of mobile screw dislocations, $l_{j}$ is jog spacing and $\sigma_{o}$ is a threshold stress. The introduction of threshold stress was justified by the experimental data that showed negative intercept of the plots of steady-state creep rate vs $\sigma$. Based on experimental results it was concluded that H-D creep in $\alpha-\mathrm{Zr}$ is controlled by the core diffusion with diffusion coefficient equal to 


$$
D_{c}=10^{-4} \exp \left(-\frac{121.8 \mathrm{~kJ} / \mathrm{mol}}{R T}\right) \frac{\mathrm{m}^{2}}{\mathrm{~s}}
$$

Novotny et al. also proposed to use Ardell et al 1965 expression to obtain equilibrium jog spacing

$$
l_{j}=\frac{b}{5} \exp \left(\frac{U_{j}}{R T}\right)
$$

with the experimentally obtained $\mathrm{U}_{\mathrm{j}}=27.2 \mathrm{~kJ} / \mathrm{mol}$, and introduced a Harper-Dorn constant equal

$$
A_{H D}=K \rho_{s} l_{j} b
$$

and which decreases with temperature. Substituting $A_{H D}$ to the creep rate equation gives:

$$
\frac{\dot{\varepsilon}}{D_{c}}=A_{H D} \frac{b}{k T}\left(\sigma-\sigma_{o}\right)
$$

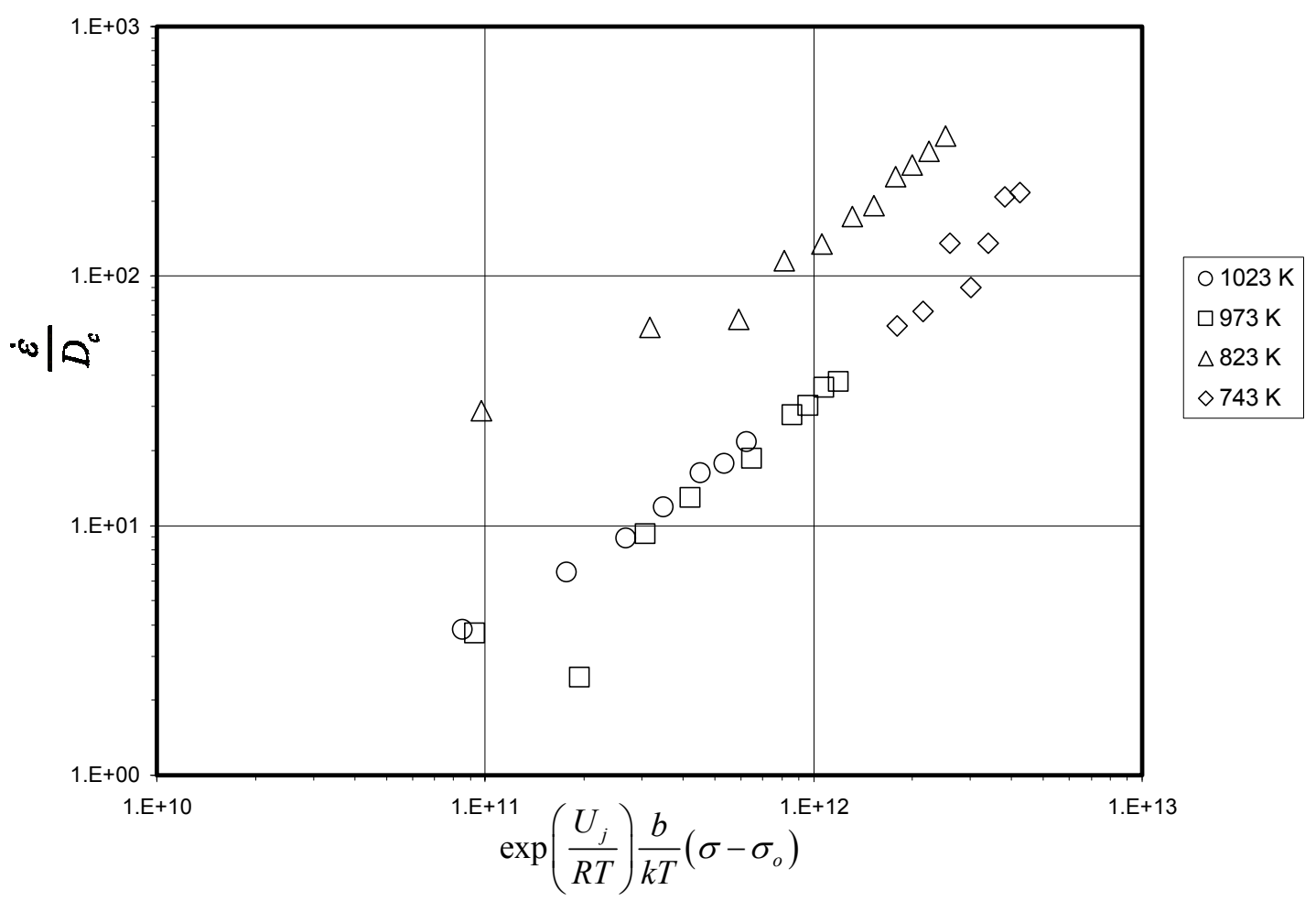

Figure 6. Comparison of the predictions of the Novotny et al 1985 H-D creep theory, eq. (1.123), with experimental data for $\alpha-Z$ r. For perfect agreement all data should fall on one straight line. 
To check the predictive capabilities of Novotny et al 1995 theory the plot of

$$
\frac{\dot{\varepsilon}}{D_{c}} v s \exp \left(\frac{U_{j}}{R T}\right) \frac{b}{k T}\left(\sigma-\sigma_{o}\right)
$$

should be a straight line with the slope $A_{H D}^{{ }_{H D}}=K / 5 \rho_{s} b^{2}$ : This plot, for the available relevant data for $\alpha-Z r$, is shown in Figure 6. With the exception for data for $823 \mathrm{~K}$ all other experimental results seems to fall on one straight line supporting the proposed model. 


\section{The Dislocation Network Theory}

The dislocation network theory of high temperature creep was originally proposed by McLean (Mitra and McLean, 1967) and subsequently put into a quantitative framework by Lagneborg and his co-workers (Lagneborg et al, 1972, 1973, 1974, Ostrom et al, 1976, 1980). Several shortcomings of the early version of that theory have been addressed by us in a series of publications (Ardell et al, 1984, 1986, 1993, 1997, Lin et al, 1989, Przystupa et al, 2000, 2002) which led to the current formulation of the dislocation network theory of the H-D creep (Przystupa et al, 2002). This theory assumes that dislocations generated upon loading form a three-dimensional Frank network that subsequently coarsens under stress, with concomitant continuous refinement by collisions between gliding links. During primary creep the coarsening dominates, which results in an increase of the average link length, $\langle\mathrm{L}\rangle$, and a reduction in the dislocation density, $\rho$. Eventually, the coarsening rate decreases due to exhaustion of the available links that can react and the network becomes frustrated (Ardell, 1997). The coarsening would then stop if not for the occasional link collisions that refine the network and thus keep the coarsening process alive. At this stage the coarsening and refinement rates balance each other and steady-state sets in. The creep strain results from movement of the links. The decreasing creep rate during primary creep and the development of the steady-state conditions are natural consequences of the slowing network growth rate. At high stresses in the power law creep regime the longest links in the network can bow out between pinning points to semicircles, hence become unstable and therefore free to glide in an unconstrained manner. If there are no such links, which we argue happens during H-D creep, the strain can be only generated by network coarsening. The transition between these two regimes takes place when the stress is just sufficient to bow out the longest links, $\mathrm{L}_{\mathrm{c}}$, to semicircle.

\section{Basic Relations}

Following Ardell et al 1984, it is assumed that the link length distribution is given by a distribution function $\phi(\mathrm{L}, \mathrm{t})$ define in such a way that $\phi(\mathrm{L}, \mathrm{t}) \mathrm{dL}$ describes the number of 
links, per unit volume, with lengths in the interval $[\mathrm{L}, \mathrm{L}+\mathrm{dL}]$. The total number of links per unit volume, $\mathrm{N}$, is then equal to

$$
N=\int_{0}^{\infty} \phi(L, t) d L
$$

Knowing the distribution function, $\phi(L, t)$, the average link length can be obtained from relation:

$$
\langle L\rangle=\frac{1}{N} \int_{0}^{\infty} L \phi(L, t) d L
$$

which gives dislocation density equal to:

$$
\rho=N\langle L\rangle=\int_{0}^{\infty} L \phi(L, t) d L
$$

Assuming additionally that each link can be associated with a volume $\mathrm{L}^{3}$, the "volume fraction" of those volumes must be equal to unity and one obtains

$$
1=\int_{0}^{\infty} C(L, t) L^{3} \phi(L, t) d L=\left\langle C(L, t) L^{3}\right\rangle N=C_{1}\langle L\rangle^{3} N
$$

where $\mathrm{C}(\mathrm{L}, \mathrm{t})$ and $\mathrm{C}_{1}$ is a geometrical factors. Combining last two equation gives

$$
\rho=\frac{1}{C_{1}\langle L\rangle^{2}} \text { or }\langle L\rangle=\frac{\beta}{\sqrt{\rho}}
$$

and

$$
N=\frac{\rho}{\langle L\rangle}=\rho^{3 / 2} / \beta
$$

where $\beta=\frac{1}{\sqrt{\mathrm{C}_{1}}}$ is a new geometrical constant. Assuming additionally that there is a critical link length $\mathrm{L}_{\mathrm{c}}$ such that links with $\mathrm{L}<\mathrm{L}_{\mathrm{c}}$ constitute network and those with $\mathrm{L}>\mathrm{L}_{\mathrm{c}}$ glide, the number of network links per unit volume, $\mathrm{N}_{\mathrm{n}}$, and network dislocation density, $\rho_{\mathrm{n}}$, are:

$$
N_{n}=\int_{0}^{L_{c}} \phi(L, t) d L \text { and } \rho_{n}=N_{n}\left\langle L_{n}\right\rangle=\int_{0}^{L_{c}} L \phi(L, t) d L
$$

Similarly, for glissile links

$$
N_{g}=\int_{L_{c}}^{\infty} \phi(L, t) d L \text { and } \rho_{g}=N_{g}\left\langle L_{g}\right\rangle=\int_{L_{c}}^{\infty} L \phi(L, t) d L
$$

The sum of network and gliding links must of course be equal to $\mathrm{N}$ and adding densities of network and glissile dislocations gives the total dislocation density, $\rho$. 


\section{Evolution of dislocation network}

The evolution of the link length distribution function, $\phi(L, t)$, with time can be described by the modified coarsening equation (Wagner 1961, Ardell et al 1984):

$$
\frac{\partial \phi}{\partial t}=-\frac{\partial(\phi g)}{\partial t}+\sum_{i=1}^{m} Q_{i}(L, t)
$$

where $g=g(L, t)=d L / d t-$ link growth rate

$\mathrm{Q}_{\mathrm{i}}(\mathrm{L}, \mathrm{t})$ - source terms describing contributions from network refinement and recovery processes

The first term on the right hand side of eq. (2.9) represent then contribution from network coarsening, with $\phi^{\prime} \mathrm{g}$ representing the flux of links leaving the interval $[\mathrm{L}, \mathrm{L}+\mathrm{dL}]$ due to coarsening. The second term is a sum of the contributions from yet undetermined network refinement and recovery processes. The source terms $\mathrm{Q}_{\mathrm{i}}(\mathrm{L}, \mathrm{t})$ in that sum are defined in such a way that $\mathrm{Q}_{\mathrm{i}}(\mathrm{L}, \mathrm{t}) \mathrm{dL}$ describes the number of links per unit volume and time contributed to interval $[\mathrm{L}, \mathrm{L}+\mathrm{dL}]$ from the $\mathrm{i}$-th link source. Knowing all quantities in eq. (2.9) it is possible to estimate rate of changes of the links number and dislocation density as

$$
\frac{d N}{d t}=\int_{0}^{\infty} \frac{\partial \phi}{\partial t} d L=-\int_{0}^{\infty} \frac{\partial(\phi g)}{\partial t} d L+\sum_{i=1}^{m} \int_{0}^{\infty} Q_{i}(L, t) d L
$$

and

$$
\frac{d \rho}{d t}=\int_{0}^{\infty} L \frac{\partial \phi}{\partial t} d L=-\int_{0}^{\infty} L \frac{\partial(\phi g)}{\partial t} d L+\sum_{i=1}^{m} \int_{0}^{\infty} L Q_{i}(L, t) d L
$$

To proceed further it will be then necessary to identified mechanisms controlling network refinement and develop expressions for the corresponding source terms, $\mathrm{Q}_{\mathrm{i}}$; this will be done in the next section.

\section{a. Network Refinement and Recovery Processes}

The network refinement and recovery processes considered in this work are shown schematically in Figure 7. Considering first network refinements, each collision between two links results in their destruction and creation of five, four or two new links. Hence, if 


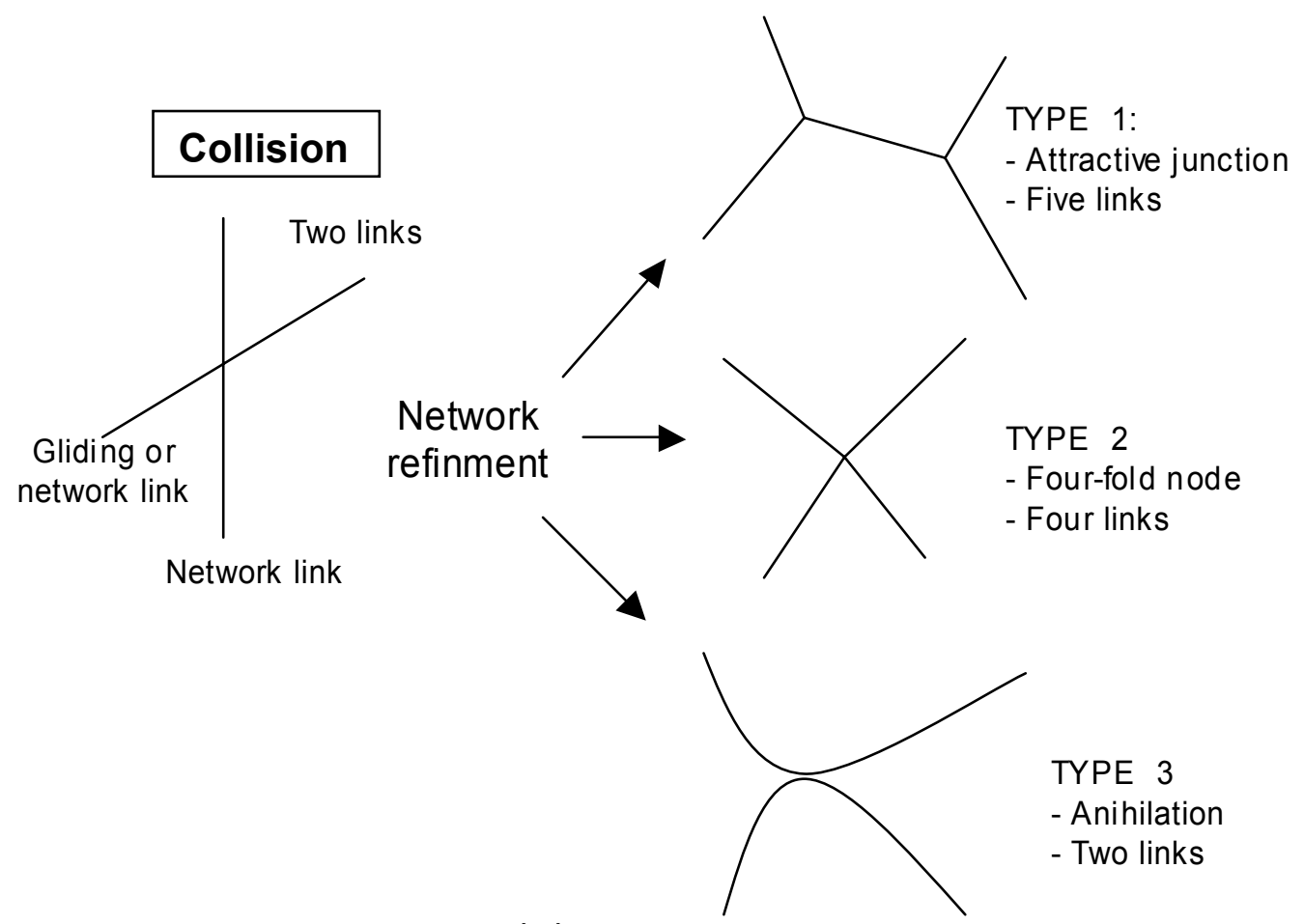

(a)

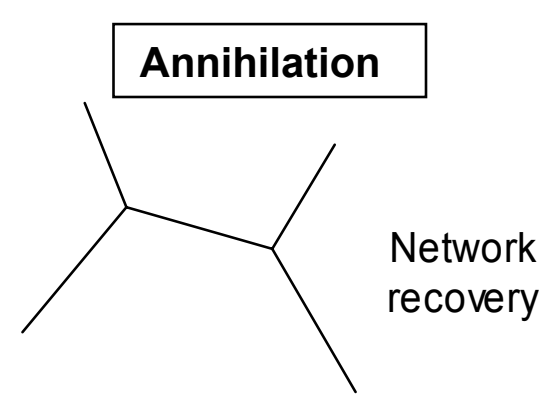

Five links
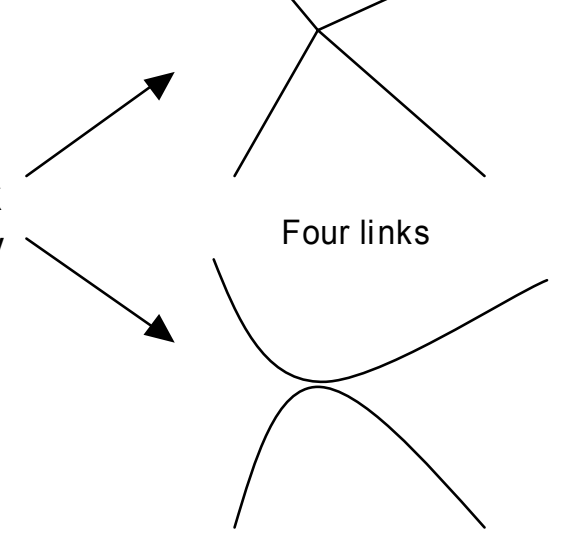

Two links

(b)

Figure 7. Schematics showing (a) network refinement and (b) recovery processes taking place during high temperature creep. 
$\mathrm{M}_{\mathrm{t}}$ is total rate of collision per unit volume between links and $\mathrm{c}_{\mathrm{i}}$ is fraction of collisions of type I, then

$3 \mathrm{c}_{1} \mathrm{M}_{\mathrm{t}}$ - rate of increase in the number of links per unit volume due to collisions of type 1

$2 c_{2} M_{t}$ - rate of increase in the number of links per unit volume due to collisions of type 2

$c_{3} 0$ - rate of increase in the number of links per unit volume due to collisions of type 3

Recovery by annihilation of one link results on the other hand in the formation of a fourfold node or in the recombination of four links into two longer ones. Consequently if $\mathrm{M}_{\mathrm{O}}$ is the link annihilation rate and $c_{0}$ is the fraction of annihilations resulting in recombinations then

$-2 \mathrm{M}_{\mathrm{O}} \mathrm{c}_{\mathrm{O}}-$ link number reduction rate due to recovery

Summing network refinement and recovery rates gives the contribution of the source terms to the rate of change the number of links excluding links shrinking to zero length. This rate is equal to:

$$
\sum_{i=1}^{m} \int_{0}^{\infty} Q_{i}(L, t) d L=M_{t}\left(3 c_{1}+2 c_{2}\right)-2 M_{o} c_{o}
$$

Also noting that for a fixed time

$$
\int_{0}^{\infty} \frac{\partial(\phi g)}{\partial L} d L=-\lim _{L \rightarrow 0}(\phi g)
$$

and substituting last two relation to eq. (2.10) gives

$$
\frac{d N}{d t}=\lim _{L \rightarrow 0}(\phi g)+M_{t}\left(3 c_{1}+2 c_{2}\right)-2 M_{o} c_{o}
$$

The rate of change of dislocation density can be obtained from eq. (2.11). The first term in that relation describes the change due to network coarsening and second that due to network refinement. In Ardell et al 1984 it was assumed that refinement by collision does not result in the change of the sum of the link lengths, thus 


$$
\sum_{i=1}^{m} \int_{0}^{\infty} L Q_{i}(L, t)=0
$$

However, for the collision and annihilation processes considered above one can expect a net change (most likely decrease) in the sum of link lengths. This change can be assumed equal to the product of the collision rate, $\mathrm{M}_{t}$, and the average change of the sum of the lengths of participating link, $\langle\Delta \mathrm{L}\rangle$, hence

$$
\sum_{i=1}^{m} \int_{0}^{\infty} L Q_{i}(L, t)=M_{t}\langle\Delta L\rangle
$$

Noting additionally that for a fixed time the first term in eq. (2.11) can be written as

$$
-\int_{0}^{\infty} L \frac{\partial(\phi g)}{\partial L} d L=-\int_{0}^{\infty} L d(\phi g)=\int_{0}^{\infty} \phi g d L=N\langle g\rangle
$$

and combining eq. (2.11) with the last two eqs. gives change of the dislocation density equal to

$$
\frac{d \rho}{d t}=N\langle g\rangle+M_{t}\langle\Delta L\rangle
$$

It is worth to note that differentiating eq. (2.3) and combining result with (2.18) gives

$$
\langle L\rangle \frac{d N}{d t}+N \frac{d\langle L\rangle}{d t}=N\langle g\rangle+M_{t}\langle\Delta L\rangle
$$

or

$$
\langle g\rangle=\left\langle\frac{d L}{d t}\right\rangle=\frac{\langle L\rangle}{N} \frac{d N}{d t}+\frac{d\langle L\rangle}{d t}-\frac{M_{t}}{N}\langle\Delta L\rangle
$$

which means that

$$
\left\langle\frac{d L}{d t}\right\rangle \neq \frac{d\langle L\rangle}{d t}
$$

Eqs. (2.12), (2.14), (2.16) and (2.19) constitute constraints on the distribution function $\phi(\mathrm{L}, \mathrm{t})$.

\section{b. Link Sources}

To obtain link length distribution function from eq. (2.9) it is necessary to identify all source term $\mathrm{Q}_{\mathrm{i}}(\mathrm{L}, \mathrm{t})$. Assuming that gliding links collide only with network links and become part of the network, the contribution from those terms to region of links smaller 
than $L_{c}$ will be different than those for links greater than $L_{c}$, as shown schematically in Figure 8. Consequently, the source terms for each of those regions will be considered separately.

\section{i. Region of $0<\mathrm{L}<\mathrm{Lc}$}

Based on the assumed network refinement and recovery processes shown in Figure 8 , there are the following seven source terms contributing to the change of the

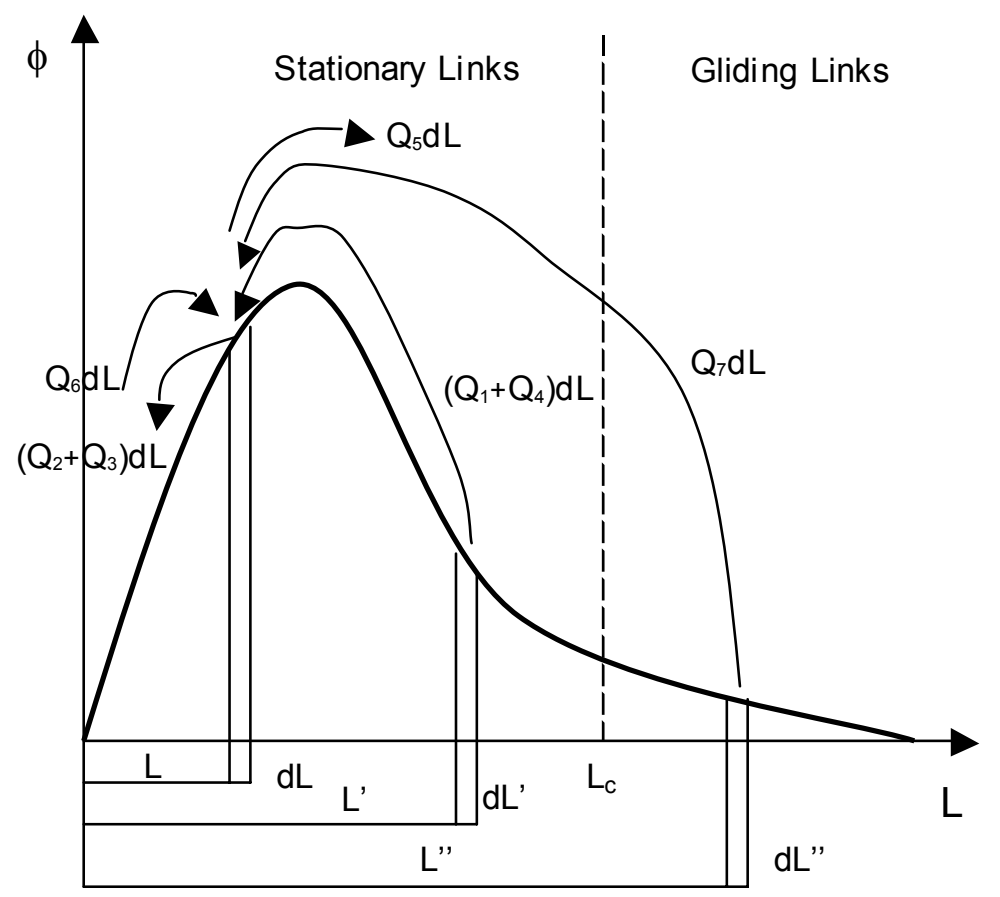

Figure 8. Schematic showing links length distribution function and contributions of source terms $\mathrm{Q}_{\mathrm{i}} \mathrm{dL}$ to the change of link number in the interval $[\mathrm{L}, \mathrm{L}+\mathrm{dL}]$. $\mathrm{L}_{\mathrm{c}}$ is a critical link length; links smaller then $\mathrm{L}_{\mathrm{c}}$ form the network and larger than $\mathrm{L}_{\mathrm{c}}$ glide.

number of links in the interval $[\mathrm{L}, \mathrm{L}+\mathrm{dL}]$ for the links in the region $0<\mathrm{L}<\mathrm{Lc}$ :

$\mathrm{Q}_{1}$ - new links produced by division of network links with lengths in interval [L', $\left.\mathrm{L}^{`}+\mathrm{dL} \mathrm{L}^{`}\right]$ by the gliding links

$\mathrm{Q}_{2}$ - links leaving interval $[\mathrm{L}, \mathrm{L}+\mathrm{dL}]$ due to collision with gliding links 
Q3 - links leaving interval $[\mathrm{L}, \mathrm{L}+\mathrm{dL}]$ due to collision with network links

Q4 - new links produced by division of network links with lengths in interval [L', $\left.\mathrm{L}^{\prime}+\mathrm{dL} \mathrm{L}^{\prime}\right]$ by other network links

Q5 - links leaving interval $[\mathrm{L}, \mathrm{L}+\mathrm{dL}]$ due to recombination

Q6 - links produced by recombination of shorter links due to the annihilation events

Q7 - links produced by division of gliding links by the network links Each of those terms will be considered in details below:

Source term $\mathbf{Q}_{\underline{1}}:$ Contribution from devision of network links with lengths in interval $\left[L^{\prime}, L^{\prime}+d L^{\prime}\right]$ by the gliding links

Assuming that $\mathrm{M}_{\mathrm{gn}}$ is the rate of collisions between gliding and network links and that $c_{\text {gni }}$ is fraction of collisions between gliding and network links of type $i$ then the number of network links destroyed per unit volume during time $\delta \mathrm{t}$ is $\mathrm{M}_{\mathrm{gn}} \delta \mathrm{t}$. Assuming additionally that distribution of those links is similar to the original distribution gives:

$$
\begin{aligned}
& \frac{\phi\left(L^{\prime}, t\right) \cdot d L^{\prime}}{N_{n}} \cdot M_{g n} \cdot \delta t-n u m b e r \text { of network links lost from interval }\left[L^{\prime}, L^{\prime}+d L^{\prime}\right] \\
& \frac{3 \cdot \phi\left(L^{\prime}, t\right) \cdot d L^{\prime}}{N_{n}} \cdot M_{g n} \cdot c_{g n 1} \cdot \delta t-\text { number of new links from collisions of type } 1 \\
& \frac{2 \cdot \phi\left(L^{\prime}, t\right) \cdot d L^{\prime}}{N_{n}} \cdot M_{g n} \cdot c_{g n 2} \cdot \delta t-\text { number of new links from collisions of type } 2 \\
& \frac{1 \cdot \phi\left(L^{\prime}, t\right) \cdot d L^{\prime}}{N_{n}} \cdot M_{g n} \cdot c_{g n 3} \cdot \delta t-\text { number of new links from collisions of type } 3
\end{aligned}
$$

For collisions of type 1 and 3 it is reasonable to assume that the size distribution of the new links will be also similar to the original distribution, hence:

$$
\begin{array}{r}
\frac{\left(3 \cdot c_{\mathrm{gn} 1}+\mathrm{c}_{\mathrm{gn} 3}\right) \cdot \phi\left(\mathrm{L}^{\prime}, \mathrm{t}\right) \cdot \mathrm{dL}}{\mathrm{N}_{\mathrm{n}}} \cdot \mathrm{M}_{\mathrm{gn}} \cdot \mathrm{dt} \cdot \frac{\phi(\mathrm{L}, \mathrm{t}) \cdot \mathrm{dL}}{\mathrm{N}_{\mathrm{n}}}-\text { number of links coming from interval [L', } \\
\left.\mathrm{L}^{\prime}+\mathrm{dL} \mathrm{L}^{\prime}\right] \text { to }[\mathrm{L}, \mathrm{L}+\mathrm{dL}] \text { as a result of collisions of type } 1 \text { and } 3
\end{array}
$$

Since the network links are intersected anywhere along their length, the probability that a new link has a length between $\mathrm{L}$ and $\mathrm{L}+\mathrm{dL}$ is uniform and equal $\mathrm{dL} / \mathrm{L}$ '. Consequently 


$$
\begin{aligned}
& \frac{2 \cdot \phi\left(L^{\prime}, t\right) \cdot d L^{\prime}}{N_{n}} \cdot M_{g n} \cdot c_{g n} \cdot \delta t \cdot \frac{d L}{L^{\prime}}-\text { number of links coming from interval }\left[L^{\prime}, L^{\prime}+d L^{\prime}\right] \text { to }[L, \\
& \mathrm{L}+\mathrm{dL}] \text { as a result of collisions of type } 2
\end{aligned}
$$

The total number of new links in interval $[\mathrm{L}, \mathrm{L}+\mathrm{dL}]$ resulting from the division of network links by the gliding links is then equal to

$$
\mathrm{Q}_{1}(\mathrm{~L}, \mathrm{t})=\frac{\mathrm{M}_{\mathrm{gn}} \cdot\left(3 \cdot \mathrm{c}_{\mathrm{gn} 1}+\mathrm{c}_{\mathrm{gn} 3}\right)}{\mathrm{N}_{\mathrm{n}}} \cdot \phi(\mathrm{L}, \mathrm{t})+\frac{2 \cdot \mathrm{M}_{\mathrm{gn}} \cdot \mathrm{c}_{\mathrm{gn} 2}}{\mathrm{~N}_{\mathrm{n}}} \cdot \int_{\mathrm{L}}^{\mathrm{L}_{\mathrm{c}}} \frac{\phi\left(\mathrm{L}^{\prime}, \mathrm{t}\right)}{\mathrm{L}^{\prime}} \mathrm{d} \mathrm{L}^{\prime}
$$

Source term $\mathbf{Q}_{2}$ : Contribution from links leaving interval $[\mathrm{L}, \mathrm{L}+\mathrm{dL}]$ due to collision with gliding links

Based assumptions made for source term $\mathrm{Q}_{1}$ the number of links leaving the interval [L, $\mathrm{L}+\mathrm{dL}]$ as a result of collision with gliding links is

$$
\mathrm{Q}_{2} \cdot \mathrm{dL}=-\frac{\mathrm{M}_{\mathrm{gn}}}{\mathrm{N}_{\mathrm{n}}} \cdot \phi(\mathrm{L}, \mathrm{t}) \cdot \mathrm{dL}
$$

Source term $\mathbf{Q}_{\mathbf{3}}$ : Contribution from links leaving interval $[\mathrm{L}, \mathrm{L}+\mathrm{dL}]$ due to collision with network links

Assuming that $\mathrm{M}_{\mathrm{nn}}$ is the rate of intersections between network link and that $\mathrm{c}_{\mathrm{nni}}$ are the fractions of network-network collisions of type i, the number of network links destroyed in this process per unit volume during time $\delta \mathrm{t}$ is $2 \mathrm{M}_{\mathrm{n}} \delta \mathrm{t}$. Making the same assumptions as for $\mathrm{Q}_{2}$ gives than the number of to links leaving interval $[\mathrm{L}, \mathrm{L}+\mathrm{dL}]$ due to collision with network links equal to

$$
\mathrm{Q}_{3} \cdot \mathrm{dL}=-\frac{2 \cdot \mathrm{M}_{\mathrm{nn}}}{\mathrm{N}_{\mathrm{n}}} \cdot \phi(\mathrm{L}, \mathrm{t}) \cdot \mathrm{dL}
$$

Source term Q4: Contribution from the division of network links with lengths in interval

$$
\left[\mathrm{L}^{\prime}, \mathrm{L}^{\prime}+\mathrm{dL} \mathrm{L}^{\prime}\right] \text { by other network links }
$$

As a result of collision between network links, the number of newly created network links in time $\delta \mathrm{t}$ is

$$
\begin{aligned}
& 5 M_{n n} c_{n n 1} \delta t \text { - due to collisions of type } 1 \\
& 4 M_{n n} c_{n n} \delta t \text { - due to collisions of type } 2 \\
& 2 M_{n n} c_{n n 3} \delta t \text { - due to collisions of type } 3
\end{aligned}
$$


Assuming as for $\mathrm{Q}_{1}$ that the distribution of those links is the same as the original one for reactions of type 1 and 3 and uniform for reactions of type 2 gives

$$
\begin{gathered}
\left(5 \cdot c_{n n 1}+2 \cdot c_{n n 3}\right) \cdot \frac{\phi(L, t) \cdot d L}{N_{n}} \cdot M_{n n} \cdot \delta t-\text { number of new links joining interval }[L, L+d L] \\
\text { from reactions of type } 1 \text { and } 3 \\
\frac{4 \cdot c_{n n 2} \cdot \phi\left(L^{\prime}, t\right) \cdot d L^{\prime}}{N_{n}} \cdot M_{n n} \cdot \delta t \cdot \frac{d L}{L^{\prime}}- \\
L+d L] \text { as a result of collisions of type } 2
\end{gathered}
$$

Consequently, the number of links coming to interval [L', $\left.\mathrm{L}^{\prime}+\mathrm{dL}^{\prime}\right]$ as a result of collision between longer network links is equal to

$$
\mathrm{Q}_{4}=\frac{\left(5 \cdot \mathrm{c}_{\mathrm{nn} 1}+2 \cdot \mathrm{c}_{\mathrm{nn} 3}\right) \cdot \mathrm{M}_{\mathrm{nn}}}{\mathrm{N}_{\mathrm{n}}} \cdot \phi(\mathrm{L}, \mathrm{t})+\frac{4 \cdot \mathrm{M}_{\mathrm{nn}} \cdot \mathrm{c}_{\mathrm{nn} 2}}{\mathrm{~N}_{\mathrm{n}}} \cdot \int_{\mathrm{L}}^{\mathrm{L}_{\mathrm{c}}} \frac{\phi\left(\mathrm{L}^{\prime}, \mathrm{t}\right)}{\mathrm{L}^{\prime}} \mathrm{dL}^{\prime}
$$

Source term Q5: Contribution from links leaving interval $[\mathrm{L}, \mathrm{L}+\mathrm{dL}]$ due to recombinations

If $\mathrm{M}_{\mathrm{O}}$ is the link annihilation rate and $\mathrm{c}_{\mathrm{O}}$ fraction of annihilation events leading to link recombination then each annihilation event results in either recombination of four links into two longer ones or formation of a four-fold node. Assuming that the distribution of destroyed links is similar to $\phi$ gives

$\frac{\phi(\mathrm{L}, \mathrm{t}) \cdot \mathrm{dL}}{\mathrm{N}} \cdot 4 \mathrm{M}_{\mathrm{o}} \cdot \mathrm{c}_{\mathrm{o}} \cdot \delta 1$ - number of destroyed links coming from interval $[\mathrm{L}, \mathrm{L}+\mathrm{dL}]$

As a result, the number of links leaving interval $[\mathrm{L}, \mathrm{L}+\mathrm{dL}]$ due to recombination is equal to

$$
\mathrm{Q}_{5} \cdot \mathrm{dL}=-\frac{4 \mathrm{M}_{\mathrm{O}} \cdot \mathrm{c}_{\mathrm{o}}}{\mathrm{N}} \cdot \phi(\mathrm{L}, \mathrm{t}) \cdot \mathrm{dL}
$$

Source term $\mathbf{Q}_{\mathbf{6}}$ : Contribution from links produced by recombination of shorter links due to the annihilation events

Assuming that the distribution of recombined links is also similar to $\phi$ one obtains $\frac{\phi(\mathrm{L}, \mathrm{t}) \cdot \mathrm{dL}}{\mathrm{N}} \cdot 2 \cdot \mathrm{M}_{\mathrm{o}} \cdot \mathrm{c}_{\mathrm{o}} \cdot \delta \mathrm{t}-$ number of recombined links joining the interval $[\mathrm{L}, \mathrm{L}+\mathrm{dL}]$ 
which gives the number of links formed by recombination of shorter links due to the annihilation equal to

$$
\mathrm{Q}_{6} \cdot \mathrm{dL}=\frac{2 \cdot \mathrm{M}_{\mathrm{o}} \cdot \mathrm{c}_{\mathrm{o}}}{\mathrm{N}} \cdot \phi(\mathrm{L}, \mathrm{t}) \cdot \mathrm{dL}
$$

Source term $\mathbf{Q}_{7}$ : Contribution from links produced by division of gliding links by the network links

To estimate the source term describing number of links joining each interval due to division of gliding links we define the two following quantities:

$\mathrm{M}_{\mathrm{d}}$ - rate of interception of gliding links by the network

$\mathrm{j}$ - average number of network links colliding with one gliding link

Hence

$$
j=\frac{M_{g n}}{M_{d}}
$$

and

$2 \mathrm{j}+1$ - number of new network links created by the division of one gliding link

$(2 \mathrm{j}+1) \mathrm{M}_{\mathrm{d}}$ - flux of new links due to division of gliding links

If the distribution of the flux links is similar to $\phi$ then number of links produced by division of gliding links by the network links is

$$
\mathrm{Q}_{7} \cdot \mathrm{dL}=\frac{(2 \cdot \mathrm{j}+1) \cdot \mathrm{M}_{\mathrm{d}}}{\mathrm{N}_{\mathrm{n}}} \cdot \phi(\mathrm{L}, \mathrm{t}) \cdot \mathrm{dL}
$$

Substituting all source terms into continuity eq.(2.9) gives

$$
\begin{aligned}
\frac{\mathrm{d}}{\mathrm{dt}} \phi= & -\frac{\mathrm{d}}{\mathrm{dL}}(\phi \cdot \mathrm{g})+\frac{\mathrm{M}_{\mathrm{gn}} \cdot\left(3 \cdot \mathrm{c}_{\mathrm{gn} 1}+\mathrm{c}_{\mathrm{gn} 3}\right)}{\mathrm{N}_{\mathrm{n}}} \cdot \phi(\mathrm{L}, \mathrm{t}) \ldots \\
& +\frac{2 \cdot \mathrm{M}_{\mathrm{gn}} \cdot \mathrm{c}_{\mathrm{gn} 2}}{\mathrm{~N}_{\mathrm{n}}} \cdot \int_{\mathrm{L}}^{\mathrm{L}_{\mathrm{c}}} \frac{\phi\left(\mathrm{L}^{\prime}, \mathrm{t}\right)}{\mathrm{L}^{\prime}} \mathrm{dL} \mathrm{L}^{\prime}-\frac{\mathrm{M}_{\mathrm{gn}}}{\mathrm{N}_{\mathrm{n}}} \cdot \phi(\mathrm{L}, \mathrm{t})-\frac{2 \cdot \mathrm{M}_{\mathrm{nn}}}{\mathrm{N}_{\mathrm{n}}} \cdot \phi(\mathrm{L}, \mathrm{t}) \ldots \\
& +\frac{\mathrm{M}_{\mathrm{nn}} \cdot\left(5 \cdot \mathrm{c}_{\mathrm{nn} 1}+2 \cdot \mathrm{c}_{\mathrm{nn} 3}\right)}{\mathrm{N}_{\mathrm{n}}} \cdot \phi(\mathrm{L}, \mathrm{t})+\frac{4 \cdot \mathrm{M}_{\mathrm{nn}} \cdot \mathrm{c}_{\mathrm{nn} 2}}{\mathrm{~N}_{\mathrm{n}}} \cdot \int_{\mathrm{L}}^{\mathrm{L}_{\mathrm{c}}} \frac{\phi\left(\mathrm{L}^{\prime}, \mathrm{t}\right)}{\mathrm{L}^{\prime}} \mathrm{dL^{ \prime }}-\frac{4 \cdot \mathrm{M}_{\mathrm{o}} \cdot \mathrm{c}_{\mathrm{o}}}{\mathrm{N}_{\mathrm{n}}} \cdot \phi(\mathrm{L}, \mathrm{t}) \ldots \\
& +\frac{2 \cdot \mathrm{M}_{\mathrm{o}} \cdot \mathrm{c}_{\mathrm{o}}}{\mathrm{N}_{\mathrm{n}}} \cdot \phi(\mathrm{L}, \mathrm{t})+\frac{(2 \cdot \mathrm{j}+1) \cdot \mathrm{M}_{\mathrm{d}}}{\mathrm{N}_{\mathrm{n}}} \cdot \phi(\mathrm{L}, \mathrm{t})
\end{aligned}
$$

which after some rearrangements reduces to 


$$
\begin{aligned}
\frac{\partial \phi}{\partial t}= & -\frac{\partial(\phi g)}{\partial L}+\frac{2}{N_{n}}\left(M_{g n} c_{g n 2}+2 M_{n n} c_{n n 2}\right) \int_{L}^{L_{c}} \frac{\phi\left(L^{\prime}, t\right)}{L^{\prime}} d L^{\prime} \\
& +\frac{\phi(L, t)}{N_{n}}\left[M_{g n}\left(3 c_{g n 1}+2 c_{n n 3}-1\right)+M_{n n}\left(5 c_{n n 1}+2 c_{n n 1}-2\right)-2 M_{o} c_{o} \frac{N_{n}}{N}+(2 j+1) M_{d}\right]
\end{aligned}
$$

This equation describes the evolution of the distribution of network links, hence links with lengths $0<\mathrm{L}<\mathrm{L}_{\mathrm{c}}$.

\section{ii. Region of $\mathrm{L}_{\underline{c}} \leq \underline{\mathrm{L}}$}

All links in that region are mobile, which means that the change of the number of links in that region can be described by the following three source terms:

Q5 - gliding links destroyed during annihilation events

Q6 - gliding links produced by recombination of shorter links during annihilation events

Q8 - lost of gliding links intercepted by the network

$\underline{\text { Source terms }} \mathbf{Q}_{\mathbf{5}}$ and $\mathbf{Q}_{\mathbf{6}}$ : Contribution from the destruction of links during annihilation events

The expression for $\mathrm{Q}_{6}$ and $\mathrm{Q}_{5}$ are, except for sign, the same as those for the region $0<\mathrm{L}<\mathrm{Lc}$, thus

$$
\begin{aligned}
& Q_{5} d L=-\frac{2 M_{o} c_{o}}{N_{g}} \phi(L, t) d L \\
& Q_{6} d L=\frac{2 M_{o} c_{o}}{N_{g}} \phi(L, t) d L
\end{aligned}
$$

Source terms Q8 : Contribution due to interception of gliding links by the network To estimate this term let us assume that $\mathrm{p}(\mathrm{L} ", \mathrm{t}) \mathrm{dL}$ " is the cumulative probability that gliding links form the interval [L", L"+dL"] are intercepted by the network and that $g_{g}$ is the gliding link growth rate. Thus

$$
\frac{p(L ", t) \cdot d L^{\prime \prime} \cdot \phi\left(L^{\prime \prime}, t\right) \cdot d L^{\prime \prime}}{\delta t}=p(L ", t) \cdot \phi\left(L^{\prime \prime}, t\right) \cdot g_{g} \cdot d L^{\prime \prime}-\text { number of gliding links leaving interval }
$$

$$
\text { [L", L"+dL"] per unit time }
$$


Consequently

$$
Q_{8} \cdot d L=-p(L, t) \cdot \phi(L, t) \cdot g_{g} \cdot d L
$$

Making additional assumption that the probability of the intercepting gliding link is proportional to the number of links of a given length gives

$$
\mathrm{Q}_{8} \cdot \mathrm{dL}=\frac{-\phi(\mathrm{L}, \mathrm{t})}{\mathrm{N}_{\mathrm{g}}} \cdot \phi(\mathrm{L}, \mathrm{t}) \cdot \mathrm{g}_{\mathrm{g}} \cdot \mathrm{dL}=\frac{(\phi(\mathrm{L}, \mathrm{t}))^{2} \cdot \mathrm{g}_{\mathrm{g}}}{\mathrm{N}_{\mathrm{g}}} \cdot \mathrm{dL}
$$

Substituting source terns to the continuity equation (2.9) gives:

$$
\frac{\partial \phi}{\partial t}=-\frac{\partial(\phi t)}{\partial L}-\frac{2 M_{o} c_{o}}{N} \phi(L, t)-p(L, t) \cdot \phi(L, t) \cdot g_{g}
$$

which describes evolution of the distribution of gliding links with lengths greater than $\mathrm{L}_{\mathrm{c}}$.

\section{Harper - Dorn Creep}

Following Ardell et al 1986 it is assumed that during Harper-Dorn creep there are no links greater than $\mathrm{L}_{\mathrm{c}}$ hence that all links are part of the network and $\mathrm{N}=\mathrm{N}_{\mathrm{n}}$. In such a case the number of gliding links, $\mathrm{N}_{\mathrm{g}}$, the rates of interception of gliding links by the network, $\mathrm{M}_{\mathrm{d}}$, and the rate of collisions between gliding and network links, $\mathrm{M}_{\mathrm{gn}}$, are zero and

$$
\mathrm{L}_{\mathrm{c}}=\mathrm{L}_{\mathrm{m}}
$$

where $\mathrm{L}_{\mathrm{m}}$ is the maximum link length . The continuity equation describing evolution of link length distribution is then the general eq. (2.29) which for the H-D creep reduces to

$$
\frac{\partial \phi}{\partial t}=-\frac{\partial(\phi g)}{\partial L}+\frac{4 M_{n n} c_{n n 2}}{N_{n}} \int_{L}^{L_{c}} \frac{\phi\left(L^{\prime}, t\right)}{L^{\prime}} d L^{\prime}+\frac{\phi(L, t)}{N_{n}}\left[M_{n n}\left(5 c_{n n 1}+2 c_{n n 1}-2\right)-2 M_{o} c_{o}\right]
$$

The change of the total number of links per unit volume and unit time can be now obtained by adding up the contributions from the network recovery and refinement processes summarized below:

\section{Contribution from}

Network recovery:

- links shrinking to zero length

- network links destroyed
$\underline{\mathrm{dN} / \mathrm{dt}}$

$$
\begin{aligned}
& -\mathrm{Mo} \\
& -4 \mathrm{M}_{\mathrm{O}} \mathrm{c}_{\mathrm{O}}
\end{aligned}
$$


- newly created network links

$$
2 \mathrm{M}_{\mathrm{O}} \mathrm{c}_{\mathrm{O}}
$$

Network refinement through collisions:

- network links destroyed

$-2 M_{n n}$

- links created from collisions of type $1 \quad 5 c_{n n 1} M_{n n}$

- links created from collisions of type $2 \quad 4 c_{n n 2} M_{n n}$

- links created from collisions of type $3 \quad 2 c_{n n 3} M_{n n}$

As a result

$$
\frac{\mathrm{d}}{\mathrm{dt}} \mathrm{N}=-\mathrm{M}_{\mathrm{o}} \cdot\left(1+2 \cdot \mathrm{c}_{\mathrm{o}}\right)+\mathrm{M}_{\mathrm{nn}} \cdot\left(-2+5 \cdot \mathrm{c}_{\mathrm{nn} 1}+4 \cdot \mathrm{c}_{\mathrm{nn} 2}+2 \cdot \mathrm{c}_{\mathrm{nn} 3}\right)
$$

An alternative way of obtaining $\mathrm{dN} / \mathrm{dt}$ is by making use of eq. (2.34) which gives

$$
\begin{aligned}
\frac{\mathrm{d}}{\mathrm{dt}} \mathrm{N}=\int_{0}^{\mathrm{L}_{\mathrm{c}}} \frac{\mathrm{d}}{\mathrm{dt}} \phi \mathrm{dL}= & -\int_{0}^{\mathrm{L}_{\mathrm{c}}} \frac{\mathrm{d}}{\mathrm{dL}}(\phi \cdot \mathrm{g}) \mathrm{dL}+\frac{4 \cdot \mathrm{M}_{\mathrm{nn}} \cdot \mathrm{c}_{\mathrm{nn} 2}}{\mathrm{~N}_{\mathrm{n}}} \cdot \int_{0}^{\mathrm{L}_{\mathrm{c}}}\left(\int_{\mathrm{L}}^{\mathrm{L}_{\mathrm{c}}} \frac{\phi\left(\mathrm{L}^{\prime}, \mathrm{t}\right)}{\mathrm{L}^{\prime}} \mathrm{dL}\right) \\
& +\frac{\mathrm{M}_{\mathrm{nn}} \cdot\left(5 \cdot \mathrm{c}_{\mathrm{nn} 1}+2 \cdot \mathrm{c}_{\mathrm{nn} 3}-2\right)-2 \cdot \mathrm{M}_{\mathrm{o}} \cdot \mathrm{c}_{\mathrm{o}}}{\mathrm{N}_{\mathrm{n}}} \cdot \int_{0}^{\infty} \phi \mathrm{dL}
\end{aligned}
$$

Utilizing eq. (2.13) and relations from Appendix 1 one obtains

$$
\frac{\mathrm{d}}{\mathrm{dt}} \mathrm{N}=\lim _{\mathrm{L} \rightarrow 0}(\phi \cdot \mathrm{g})+4 \cdot \mathrm{M}_{\mathrm{nn}} \cdot \mathrm{c}_{\mathrm{nn} 2}+\mathrm{M}_{\mathrm{nn}} \cdot\left(5 \cdot \mathrm{c}_{\mathrm{nn} 1}+2 \cdot \mathrm{c}_{\mathrm{nn} 3}-2\right)-2 \cdot \mathrm{M}_{\mathrm{o}} \cdot \mathrm{c}_{\mathrm{o}}
$$

Since the first right-hand term in that equation is equal to the link annihilation rate, $\mathrm{M}_{0}$, then the equation reduces to

$$
\frac{\mathrm{d}}{\mathrm{dt}} \mathrm{N}=-\mathrm{M}_{\mathrm{o}} \cdot\left(1+2 \cdot \mathrm{c}_{\mathrm{o}}\right)+\mathrm{M}_{\mathrm{nn}} \cdot\left(-2+5 \cdot \mathrm{c}_{\mathrm{nn} 1}+4 \cdot \mathrm{c}_{\mathrm{nn} 2}+2 \cdot \mathrm{c}_{\mathrm{nn} 3}\right)
$$

which, as expected, is identical to eq. (2.35).

The rate of change of the dislocation density, $\mathrm{d} \rho / \mathrm{dt}$, can be also obtained with the help of eq. (2.34) as

$$
\begin{aligned}
\frac{\mathrm{d}}{\mathrm{dt}} \rho=\int_{0}^{\infty} \mathrm{L} \cdot \frac{\mathrm{d}}{\mathrm{dt}} \phi \mathrm{dL}= & -\int_{0}^{\infty} \mathrm{L} \cdot \frac{\mathrm{d}}{\mathrm{dL}}(\phi \cdot \mathrm{g}) \mathrm{dL}+\frac{4 \cdot \mathrm{M}_{\mathrm{nn}} \cdot \mathrm{c}_{\mathrm{nn} 2}}{\mathrm{~N}_{\mathrm{n}}} \cdot \int_{0}^{\infty} \mathrm{L} \cdot\left(\int_{\mathrm{L}}^{\mathrm{L}_{\mathrm{c}}} \frac{\phi\left(\mathrm{L}^{\prime}, \mathrm{t}\right)}{\mathrm{L}^{\prime}} \mathrm{dL} \mathrm{L}^{\prime}\right) \mathrm{dL} \ldots \\
& +\frac{\mathrm{M}_{\mathrm{nn}} \cdot\left(5 \cdot \mathrm{c}_{\mathrm{nn} 1}+2 \cdot \mathrm{c}_{\mathrm{nn} 3}-2\right)-2 \cdot \mathrm{M}_{\mathrm{o}} \cdot \mathrm{c}_{\mathrm{o}}}{\mathrm{N}_{\mathrm{n}}} \cdot \int_{0}^{\infty} \mathrm{L} \cdot \phi \mathrm{dL}
\end{aligned}
$$


Taking advantage of eqs. (2.7) and (2.17) and relations from Appendix1 leads to:

$$
\begin{aligned}
\frac{d \rho}{d t} & =N_{n}\left\langle g_{n}\right\rangle+2 M_{n n} c_{n n 2}\left\langle L_{n}\right\rangle+\left[M_{n n}\left(5 c_{n n 1}+2 c_{n n 3}-2\right)-2 M_{o} c_{o}\right]\left\langle L_{n}\right\rangle \\
& =N_{n}\left\langle g_{n}\right\rangle+\left\langle L_{n}\right\rangle\left[3 M_{n n} c_{n n 1}-2 M_{o} c_{o}\right]
\end{aligned}
$$

The change in the dislocation density can be also expressed as sum of the contributions from:

- Coarsening

- Cumulative change of link lengths during collisions

$$
\begin{gathered}
N_{n}\left\langle g_{n}\right\rangle \\
M_{n n}\langle\Delta L\rangle
\end{gathered}
$$

This gives the following alternative form of the expression for the rate of change of the dislocation density

$$
\frac{d \rho}{d t}=N_{n}\left\langle g_{n}\right\rangle+M_{n n}\langle\Delta L\rangle
$$

Comparing eq (2.39) and (2.40) results in the condition:

$$
\left\langle L_{n}\right\rangle\left(3 M_{n n} c_{n n 1}-2 M_{o} c_{o}\right)=M_{n n}\langle\Delta L\rangle
$$

Consequently, if it is assumed that the dislocation reactions do not result in the change of the dislocation length, then $\Delta L=0$ and

$$
\mathrm{M}_{\mathrm{nn}}=\frac{2 \cdot \mathrm{M}_{\mathrm{o}} \cdot \mathrm{c}_{\mathrm{o}}}{3 \cdot \mathrm{c}_{\mathrm{nn} 1}}
$$

Otherwise

$$
\langle\Delta L\rangle=\frac{\left\langle L_{n}\right\rangle}{M_{n n}}\left(3 M_{n n} c_{n n 1}-2 M_{o} c_{o}\right)
$$

which relates average change of the link length to the annihilation and collision rates.

\section{a. Generalization}

The form of eq, (2.34) suggests that it can be simplified by making the following generalizing assumptions:

- Distribution of links lost due to the network-network collisions and annihilation events is proportional to the current link-length distribution and described by the source term

$$
Q_{\text {lost } 1}=b_{1}(t) \phi(L, t)
$$


- New links formed during network-network collisions and link annihilation events form two distributions:

1 - proportional to the current one with the source term

$$
Q_{\text {gen } 1}=b_{2}(t) \phi(L, t)
$$

2 - uniform, having the source term equal

$$
Q_{\text {gen } 2}=A(t) \int_{0}^{L c} \frac{\phi(L, t)}{L} d L
$$

As a result, the continuity equation for the link-length distribution can be written as:

$$
\frac{\partial \phi(L, t)}{\partial t}=-\frac{d \phi(L, t) g(L, t)}{d L}+A(t) \int_{L}^{\infty} \frac{\phi(L, t)}{L} d L+B(t) \phi(L, t)
$$

where $B(t)=-b_{1}(t)+b_{2}(t)$. The unknown functions $A(t)$ and $B(t)$ can be expressed as a function of the link number and dislocation density rates $d N / d t$ and $d \rho / d t$. For that purpose let us first estimate $d N / d t$ using terms from eq. (2.45):

$$
\begin{aligned}
\frac{d N}{d t} & =\int_{0}^{\infty} \frac{d \phi(L, t)}{d t} d L \\
& =-\int_{0}^{\infty} \frac{d \phi(L, t) g(L, t)}{d t} d L+A(t) \int_{0}^{\infty}\left(\int_{L}^{\infty} \frac{\phi\left(L^{\prime}, t\right)}{L^{\prime}} d L^{\prime}\right) d L+B(t) \int_{0}^{\infty} \phi(L, t) d L \\
& =\lim _{L \rightarrow 0}(\phi(L, t) g(L, t))+A(t) N+B(t) N \\
& =-M_{o}(t)+A(t) N+B(t) N
\end{aligned}
$$

Combining next expression for $d \rho / d t$ with continuity eq. (2.45) results in:

$$
\begin{aligned}
\frac{d \rho}{d t} & =\int_{0}^{\infty} L \frac{d \phi(L, t)}{d t} d L \\
& =-\int_{0}^{\infty} L \frac{d \phi(L, t) g(L, t)}{d t} d L+A(t) \int_{0}^{\infty} L\left(\int_{L}^{\infty} \frac{\phi\left(L^{\prime}, t\right)}{L^{\prime}} d L^{\prime}\right) d L+B(t) \int_{0}^{\infty} L \phi(L, t) d L \\
& =N\langle g(t)\rangle+A(t) \frac{1}{2} N\langle L\rangle+B(t) N\langle L\rangle \\
& =N\langle g(t)\rangle+A(t) \frac{1}{2} \rho+B(t) \rho
\end{aligned}
$$

The two final expressions in (2.46) and (2.47) can be solved for $\mathrm{A}(\mathrm{t})$ and $\mathrm{B}(\mathrm{t})$ giving: 


$$
\begin{aligned}
& A(t)=\frac{2}{\rho}\left(\langle L\rangle\left(\dot{N}+M_{o}(t)\right)-\dot{\rho}+N\langle g(t)\rangle\right) \\
& B(t)=\frac{2}{\rho}\left(\dot{\rho}-N\langle g(t)\rangle-\frac{\langle L\rangle}{2}\left(\dot{N}+M_{o}(t)\right)\right)
\end{aligned}
$$

which after making reasonable assumption that

$$
\frac{d\langle L\rangle}{d t}=\left\langle\frac{d L}{d t}\right\rangle=\langle g(t)\rangle
$$

simplify to

$$
\begin{aligned}
& A(t)=\frac{2 M_{o}(t)}{N} \\
& B(t)=\frac{1}{N}\left(\frac{d N}{d t}-M_{o}(t)\right)
\end{aligned}
$$

$A(t)$ represents then the fraction of links shrinking, per unit time, to zero length and $B(t)$ the fraction of links forming, also per unit time, as a result of the reactions of type 2 and 3, Figure 7. Equations (2.48) can be now substituted to (2.34) giving the following general form of the continuity equation:

$$
\frac{\partial \phi(L, t)}{\partial t}=-\frac{\partial \phi(L, t) g(L, t)}{\partial L}+\frac{2 M_{o}(t)}{N} \int_{L}^{\infty} \frac{\phi(L, t)}{L} d L+\frac{1}{N}\left(\frac{d N}{d t}-M_{o}(t)\right) \phi(L, t)
$$

Note that from all the functions defined above only $\langle g(t)\rangle$ and $M_{o}(t)$ cannot be obtained from the links-length distribution function $\phi(L, t)$. As it will be shown below, it is possible to obtain an explicit relation for the link growth rate, $g(L, t)$, but the link annihilation rate, $M_{o}(t)$, needs to be obtained from the experimental strain rate or link length distribution data (Ardell et al 1986).

\section{b. Link Growth Rate $g(L, t)$}

The equation for $g(L, t)$ needs to be consistent with the requirements that the number of links shrinking to zero length as well of those growing beyond current maximum length are greater than zero and finite. This means that fluxes of links, given by product $\phi(\mathrm{L}, \mathrm{t})^{\cdot} \mathrm{g}(\mathrm{L}, \mathrm{t})$, must be also finite at both ends of the distribution function. Experimental data on the dislocation link length distributions for $\mathrm{Al}$ and $\mathrm{NaCl}$ analyzed 
by Lin et al. 1989 suggest that for high temperature creep $\phi(\mathrm{L}, \mathrm{t}) \rightarrow \mathrm{L}^{\mathrm{m}}$, as $\mathrm{L} \rightarrow 0$ where $\mathrm{m}$ is a constant with the value of $\approx 4 / 3$ for $\mathrm{Al}$ and $3 / 2$ for $\mathrm{NaCl}$. This means the if the product $\phi(L, t) \cdot g(L, t)$ were to remain finite in the limit of $L \rightarrow 0$ it would be necessary that $\mathrm{g}(\mathrm{L}, \mathrm{t}) \rightarrow \mathrm{L}^{-\mathrm{m}}$ in the same limit. Borrowing from the coarsening theories (Wagner 1961) a plausible empirical equation for $g(L, t)$ that fulfills those requirement is (Przystupa et al, 2002)

$$
g(L, t) \equiv \frac{d L}{d t}=v_{n}\left(\frac{L^{*}}{L}\right)^{m}\left[\frac{L}{L^{*}}-1\right]
$$

where $L^{*}(t)$ is a critical link length and $v_{n}$ is the velocity of the dislocation nodes in the network. As in the coarsening theory links with length $\mathrm{L}=\mathrm{L}^{*}$ are momentarily stationary in time; links greater than $\mathrm{L}^{*}$ grow while those smaller than $\mathrm{L}^{*}$ shrink and disappear. Eq, (2.51) is not unique and it is similar to the one proposed by Lagneborg 1972 (he used $\mathrm{m}=1$ ). However, it has advantage of scaling $\mathrm{L}$ by a natural length scale of the system, namely $\mathrm{L}^{*}$, and coupling the coarsening of the network to the velocities of the network nodes. The node velocity is in this case given by eq. (1.32), where the main contribution to the force $\mathrm{F}$ driving the motion of the nodes comes from the line tensions of the 3 dislocations meeting at the node (Evans et al 1977). This force is then equal to

$$
\mathrm{F} \approx \frac{\Gamma}{\langle\mathrm{L}\rangle}
$$

where $\Gamma$ is the dislocation line tension. Combining eqs (1.32) and (2.52) gives node velocity equal to

$$
\mathrm{v}_{\mathrm{n}}=\frac{4 \pi \mathrm{bD} \Gamma}{\mathrm{k}_{\mathrm{B}} \mathrm{T}\langle\mathrm{L}\rangle}
$$

which leads to the link growth rate equal to

$$
\mathrm{g}(\mathrm{L}, \mathrm{t})=\frac{\mathrm{dL}}{\mathrm{dt}}=\frac{4 \pi \mathrm{bD} \Gamma}{\mathrm{k}_{\mathrm{B}} \mathrm{T}\langle\mathrm{L}\rangle}\left(\frac{\mathrm{L}^{*}}{\mathrm{~L}}\right)^{\mathrm{m}}\left[\frac{\mathrm{L}}{\mathrm{L}^{*}}-1\right] .
$$


Introducing a normalized link length, $\mathrm{u}$, defined as

$$
u=\frac{L}{L^{*}(t)}
$$

the equation for link growth rate transforms into

$$
\mathrm{g}(\mathrm{L}, \mathrm{t})=\frac{\mathrm{dL}}{\mathrm{dt}}=\frac{\mathrm{k}}{\mathrm{L}^{*}} \frac{(\mathrm{u}-1)}{\mathrm{u}}
$$

where $\mathrm{k}$ is the rate constants for the kinetics of network coarsening, equal to

$$
\mathrm{k}=\frac{4 \pi \mathrm{bD} \Gamma}{\mathrm{k}_{\mathrm{B}} \mathrm{T}\langle\mathrm{u}\rangle}
$$

and $\langle u\rangle$ is equal to $\langle L\rangle / \mathrm{L}^{*}$. Note that since $\langle u\rangle$ should be a material constant, $\mathrm{k}$ should be independent of stress. This leads to the important conclusion that there should be no difference between the kinetics of network coarsening under stress during creep and that during stress-free conditions of static recovery. Borrowing again from the theory of the coarsening of precipitates (Lifshitz et al 1959, Hillert 1965) it is possible to obtain the law governing the growth of $\mathrm{L}^{*}$. For that purpose let us estimate rate of growth of the normalized link length

$$
\begin{aligned}
\frac{d u}{d t} & =\frac{d}{d t}\left(\frac{L}{L^{*}}\right)=\frac{1}{L^{*}}\left(\frac{d L}{d t}-\frac{L}{L^{*}} \frac{d L^{*}}{d t}\right) \\
& =\frac{1}{L^{*}}\left(\frac{k}{L^{*}} \frac{u-1}{u^{m}}-u \frac{d L^{*}}{d t}\right)
\end{aligned}
$$

Multiplying both sides of last expression by $\mathrm{dt} / \mathrm{dL}^{*} \cdot \mathrm{L}^{*}$ gives

$$
L^{*} \frac{d u}{d L^{*}}=\frac{k}{L^{*}} \frac{d t}{d L^{*}} \frac{u-1}{u^{m}}-u
$$

which after substitutions

$$
\begin{aligned}
& \tau=\ln L^{*} \\
& \gamma=\frac{k}{L^{*}} \frac{d t}{d L^{*}}
\end{aligned}
$$

transforms to

$$
\frac{d u}{d \tau}=\gamma \frac{u-1}{u^{m}}-u
$$


Since during coarsening $\mathrm{L}^{*}$ increases monotonically with time, $\tau$ represents here a measure of time. Following reasoning given by Lifshitz et al 1959, the link growth process described by eq. (2.61) asymptotically approaches steady state where $\gamma$ is constant and independent on the initial shape of the distribution function. It is possible to show (Lifshitz et al 1959, Hillert 1965) that the only possible value of $\gamma_{0}$ at the steady state is the one for which both growth rate $d u / d \tau$ and its derivative with respect to $u$ are equal to zero. This gives a set of equations

$$
\begin{aligned}
& \frac{d^{2} u}{d \tau d u}=\gamma\left(\frac{1}{u^{m}}-m \frac{u-1}{u^{m+1}}\right)-1 \equiv 0 \\
& \frac{d u}{d \tau}=\gamma \frac{u-1}{u^{m}}-u \equiv 0
\end{aligned}
$$

which solved for the steady state values of $\gamma_{o}$ and $u_{o}$ produce

$$
\gamma_{o}=\frac{(m+1)^{m+1}}{m^{m}} \text { and } u_{o}=\frac{m+1}{m}
$$

Substituting expression for $\gamma_{o}$ to eq. (2.60) for $\gamma$ gives differential equation governing growth of $\mathrm{L}^{*}$ hence the coarsening rate in the form

$$
L * \frac{d L^{*}}{d t}=k \frac{m^{m}}{(m+1)^{m+1}}
$$

Solving for $\mathrm{L}^{*}$ leads to link growth law

$$
L^{* 2}-L_{o}^{* 2}=\frac{2 m^{m}}{(m+1)^{m+1}} k t
$$

where $L_{o}^{*}$ is the initial value of $L^{*}$. Utilizing relations (2.5) and (2.55) we can write

$$
L^{*}=\frac{\langle L\rangle}{\langle u\rangle}=\frac{1}{\langle u\rangle} \frac{\beta}{\sqrt{\rho}}
$$

which combined with eq. (2.65) produces the following expression for the change of dislocation density during network coarsening process

$$
\frac{1}{\rho}-\frac{1}{\rho_{o}}=\kappa t
$$

where $\rho_{o}$ is the initial dislocation density and the rate constant $\kappa$ is equal to 


$$
\kappa=\left(\frac{\langle u\rangle}{\beta}\right)^{2} \frac{2 m^{m}}{(m+1)^{m+1}} k
$$

Since $\langle u\rangle, \beta$ and $m$ are material constants that can be estimated from the link-length distribution data, eq. (2.68) can be used to find $\kappa$ from the static recovery data on the change of the dislocation density with time. However, eq. (2.67) is expected to be valid only during initial stages of static recovery because, as discussed by Ardell 1997, at longer recovery times the network becomes frustrated and there is no provision for that process in the theory. It is also worth mentioning that although eq. (2.67) is derived based on the kinetics of node movement and network coarsening, it has identical form as the equation obtained by Li 1966 in his treatment of the static recovery as a second-order kinetic process.

The value of $\mathrm{L}^{*}$ can be estimated from the condition that link flux, $J(L, t)$, equal to

$$
J(L, t)=\phi(L, t) g(L, t)
$$

is zero for $\mathrm{L}=\mathrm{L}^{*}$. Following procedure developed by Ardell et al 1986 the flux can be obtained from the continuity equation $(2.50)$ by rewriting it as

$$
\frac{\partial J(L, t)}{\partial L}=-\frac{\partial \phi(L, t)}{\partial t}+\frac{2 M_{o}(t)}{N} \int_{L}^{\infty} \frac{\phi(L, t)}{L} d L+\frac{1}{N}\left(\frac{d N}{d t}-M_{o}(t)\right) \phi(L, t)
$$

For a fixed time this equation can be integrated and with the help of relations from Appendix 1 one obtains

$$
\begin{aligned}
J(L, t) & =-\frac{d}{d t} \int_{0}^{L} \phi\left(L^{\prime}, t\right) d L^{\prime}+\frac{2 M_{o}(t)}{N} L \int_{L}^{\infty} \frac{\phi\left(L^{\prime}, t\right)}{L^{\prime}} d L^{\prime} \\
& +\frac{1}{N} \frac{d N}{d t} \int_{0}^{L} \phi\left(L^{\prime}, t\right) d L^{\prime}-\frac{M_{o}(t)}{N} \int_{L}^{\infty} \phi\left(L^{\prime}, t\right) d L^{\prime}
\end{aligned}
$$

For $\mathrm{L}=\mathrm{L}^{*}$ the flux becomes zero and $\mathrm{L}^{*}$ can be obtained by numerically solving the relation:

$$
\begin{aligned}
0 & =-\frac{d}{d t} \int_{0}^{L^{*}} \phi\left(L^{\prime}, t\right) d L^{\prime}+\frac{2 M_{o}(t)}{N} L^{*} \int_{L^{*}}^{\infty} \frac{\phi\left(L^{\prime}, t\right)}{L^{\prime}} d L^{\prime} \\
& +\frac{1}{N} \frac{d N}{d t} \int_{0}^{L^{*}} \phi\left(L^{\prime}, t\right) d L^{\prime}-\frac{M_{o}(t)}{N} \int_{L^{*}}^{\infty} \phi\left(L^{\prime}, t\right) d L^{\prime}
\end{aligned}
$$

At steady-state all time derivatives become equal zero and the equations reduces to 


$$
0=2 L^{*} \int_{L^{*}}^{\infty} \frac{\phi\left(L^{\prime}, t\right)}{L^{\prime}} d L^{\prime}-\int_{L^{*}}^{\infty} \phi\left(L^{\prime}, t\right) d L^{\prime}
$$

This relation can be used to obtain $\mathrm{L}^{*}$ from the experimental data on the steady state link length distribution.

\section{c. Scaling Relations}

Eq. (2.55) defines a time-invariant measure of the link lengths which can be used to obtain a time-invariant scaled probability distribution function, $\Phi(\mathrm{u})$, through relation

$$
\Phi(u) d u=\phi(L, t) \frac{d L}{N} \quad \text { or } \quad \Phi(u)=\frac{L^{*}}{N} \phi(L, t)
$$

To show that $\Phi(\mathrm{u})$ is indeed a true probability distribution, we integrate the first of the above relations to obtain the cumulative probability

$$
\int_{0}^{\infty} \Phi(u) d u=\frac{1}{N} \int_{0}^{\infty} \phi(L, t) d L=1
$$

which, as expected, is equal to one. Eq. (2.3) can be now used to express dislocation density in terms of $\langle u\rangle$ as

$$
\begin{aligned}
\rho & =\int_{0}^{\infty} L \phi(L, t) d L=L * N \int_{0}^{\infty} u \Phi(u) d u \\
& =L^{*} N\langle u\rangle
\end{aligned}
$$

Defining scaled dimensionless links growth rate as

$$
G(u)=\frac{u-1}{u^{m}}
$$

and utilizing eq. (2.56) gives the following relation between $\mathrm{G}(\mathrm{u})$ and $\mathrm{g}(\mathrm{L}, \mathrm{t})$

$$
G(u)=\frac{L^{*}}{k} g(L, t)
$$

The link annihilation rate, $\mathrm{M}_{\mathrm{o}}$, can be also express in terms of scaled quantities as

$$
\begin{aligned}
& M_{o}(t)=-\lim _{L \rightarrow 0}\{\phi(L, t) \cdot g(L, t)\} \\
& =-\frac{N k}{L^{2}} \lim _{u \rightarrow 0}\{\Phi(u) \cdot G(u)\}
\end{aligned}
$$

Letting scaled link annihilation rate, $\mathrm{M}_{\text {ou }}$, to be equal to

$$
M_{\text {ou }}=-\lim _{u \rightarrow 0}\{\Phi(u) \cdot G(u)\}
$$


one obtains

$$
M_{o u}=\frac{L^{2}}{N k} M_{o}(t)
$$

Following our previous work (Ardell et al 1986 and Przystupa et al 2000 and 2002) the standard, $\mathrm{J}(\mathrm{L}, \mathrm{t})$, and scaled, $\vartheta(\mathrm{u})$, fluxes of links are equal to

$$
J(L, t)=\phi(L, t) \cdot g(L, t) \quad \text { and } \quad \vartheta(u)=\Phi(u) \cdot G(u)
$$

hence, similarly to eq. (2.81), one obtains

$$
\vartheta(u)=\frac{L^{* 2}}{N k} J(L, t)
$$

\section{(a) Link-length distribution}

Substituting obtained scaling relationship to the link continuity equation (2.50) transforms it to

$$
0=-\frac{d \Phi(u) G(u)}{d u}+2 M_{\text {ou }} \int_{u}^{\infty} \frac{\Phi\left(u^{\prime}\right)}{u^{\prime}} d u^{\prime}+\Phi(u)\left\{\frac{L^{*}}{k} \frac{d L^{*}}{d t}-M_{\text {ou }}\right\}
$$

Based on eq. (2.60) the first term in the last bracket is equal to $1 / \gamma$ which, as discussed in the proceeding section, is a time independent constant. Since all other coefficients in eq. (2.84) are also time independent, the probability distribution function $\Phi(\mathrm{u})$ is truly time invariant. This is a significant finding that is reported here for the first time and which gives credence to our previous experimental results on the time independence of the scaled link length distribution functions (Lin et al 1989 and Przystupa et al 2000). Equation (2.84) is then a general differential equation describing evolution of the dislocation link length distribution during both primary and steady state H-D creep.

\section{(b) Link flux}

Equation (2.84) can be significantly simplified if it is written it terms of the scaled link flux, $\vartheta(u)$, rather then the probability distribution function $\Phi(u)$. For that purpose eq. (2.84) needs to be differentiate with respect to $u$ which leads to the result

$$
(\mathrm{u}-1)^{2} \frac{\mathrm{d}^{2} \vartheta(\mathrm{u})}{\mathrm{du}^{2}}+\mathrm{M}_{\mathrm{ou}} \mathrm{u}^{\mathrm{m}}(\mathrm{u}-1) \frac{\mathrm{d} \vartheta(\mathrm{u})}{\mathrm{du}}+\mathrm{M}_{\mathrm{ou}} \mathrm{u}^{\mathrm{m}-1}[(\mathrm{~m}+1)(\mathrm{u}-1)-1] \vartheta(\mathrm{u})=0
$$


where it was additionally assumed that, as expected, $1 / \gamma$ is much less than $\mathrm{M}_{\text {ou }}$. This second order differential equation can be solved for $\vartheta(\mathrm{u})$ with boundary conditions requiring that $\vartheta(\mathrm{u})=\mathrm{M}_{\mathrm{ou}}$ and $\vartheta(1)=0$. The distribution function $\Phi(\mathrm{u})$ can be subsequently obtained from relation (2.82).

\section{(d) Verification}

The predictions of the network theory with respect to the link distribution function were tested by Przystupa et al, (2000 and 2002) by comparing its predictions with the experimental link length distributions obtained during the Harper-Dorn creep of Al single crystals at $920 \mathrm{~K}$ under applied stresses of $0.05,0.06$ and $0.08 \mathrm{MPa}$ (Ardell et al, 1986, Lin et al, 1989). Starting with the distribution functions, the values of $\mathrm{L}^{*}$ were initially determined from eq. (2.73) and then used to estimate $\langle\mathrm{u}\rangle$ and an "experimental" normalized distribution function $\Phi_{\exp }(\mathrm{u})$ via eqs. (2.55) and (2.74). An "experimental" normalized flux, $\vartheta_{\exp }(u)$, were then estimated utilizing eqs. $(2.77)$ and $(2.82)$, with $\mathrm{m}=$ 4/3 obtained by Lin et al. 1989 from analysis of the data on Al (Ardell et al, 1986). They were used to obtain the values of normalized annihilation rate $\mathrm{M}_{\mathrm{ou}}$ for each stress which

Table 2. Compilation of the data on the link length distributions and values of the parameters used in the modeling of the H-D creep of Al single crystals tested in compression at $920 \mathrm{~K}$ under the stresses indicated (Przystupa et al 2002).

\begin{tabular}{ccccccc}
\hline$\sigma(\mathrm{MPa})=$ & 0.05 & 0.06 & 0.08 & Average & St.Dev. & Validity \\
\hline \hline $\mathrm{N}\left(\mathrm{mm}^{-3}\right)$ & 1045.5 & 987.4 & 878.8 & 970.5 & 84.6 & \\
$\langle\mathrm{~L}\rangle(\mathrm{mm})$ & 0.0826 & 0.0841 & 0.0872 & 0.0846 & 0.0024 & Steady \\
$\rho\left(\mathrm{mm}^{-2}\right)$ & 86.32 & 83.00 & 76.61 & 81.98 & 4.98 & state \\
$\mathrm{L}^{*}(\mathrm{~mm})$ & 0.0403 & 0.0400 & 0.0423 & 0.0408 & 0.0012 & \\
\hline$\langle\mathrm{u}\rangle$ & 2.058 & 2.108 & 2.068 & 2.078 & 0.0273 & Primary \\
$\beta$ & 0.767 & 0.766 & 0.763 & 0.7653 & 0.0021 & and steady \\
$\mathrm{M}_{\mathrm{ou}}$ & 0.556 & 0.509 & 0.611 & 0.559 & 0.051 & state region \\
\hline
\end{tabular}


allowed for the analysis of the steady state H-D creep data. The reason for using the steady state as the starting point is that eq. (2.84) is exact under these conditions, hence $\langle\mathrm{u}\rangle$ and the function $\Phi(\mathrm{u})$ are predicted with the highest accuracy. The estimated values of $\langle\mathrm{u}\rangle$ and other characteristics of the distribution functions are summarized in Table 2. The values of $\langle\mathbf{u}\rangle$ are remarkably similar for all stresses, the maximum deviation from the average not exceeding $1.5 \%$. It can be also seen that $\mathrm{M}_{\mathrm{ou}}$ can be regarded as a stressindependent constant with best estimate of $\mathrm{M}_{\mathrm{ou}}$ for all three stresses equl to 0.577. Comparison of the predicted and experimentally measured normalized distribution functions, for both steady-state and primary creep are shown in Figs. 2(b) and 2(c).

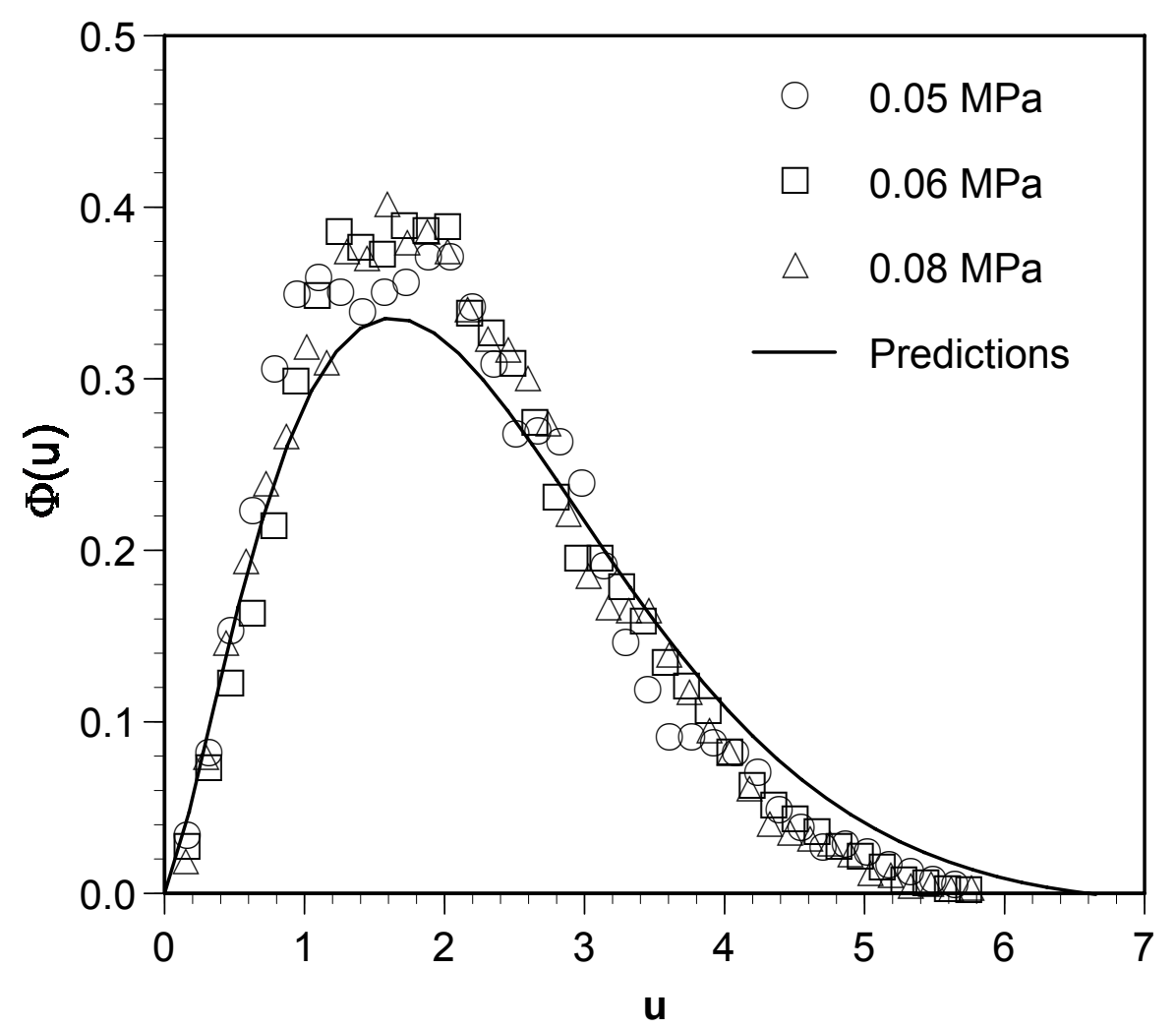

Figure 9. Scaling behavior of the normalized dislocation link-length distributions for pure Al samples during steady-state creep at indicated stresses and $913 \mathrm{~K}(0.98$ $\left.\mathrm{T}_{\text {melt }}\right)$. 


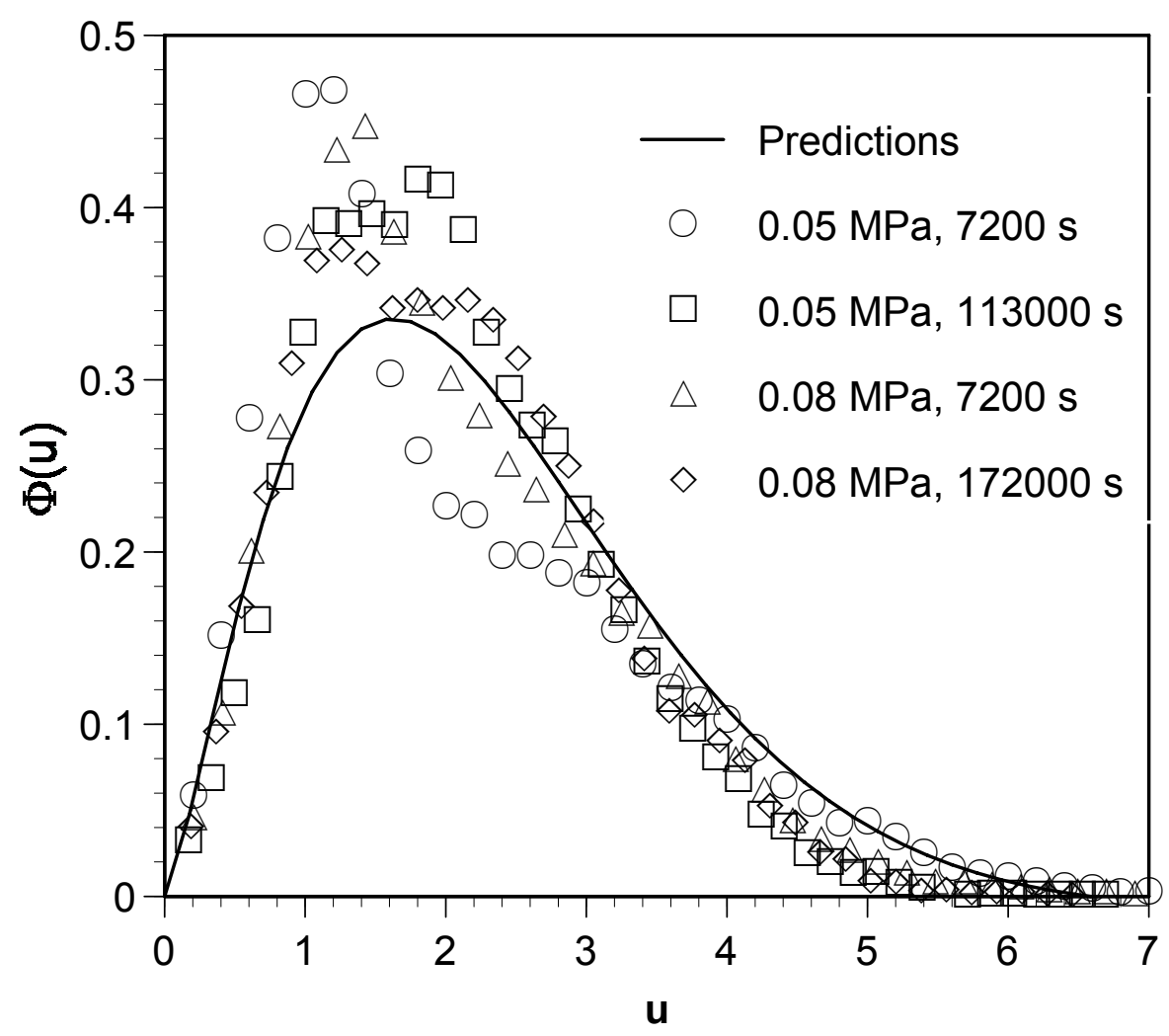

Figure 10. Scaling behavior of the normalized dislocation link-length distributions for pure Al samples during primary creep at indicated stresses and times at $913 \mathrm{~K}$

The predicted distribution function was obtained by solving numerically eq. (2.85) with exponent $m=4 / 3$ and link annihilation rate $M_{o u}=0.577$. The agreement between theory and experiment is quite good, though the theoretically calculated $\Phi(\mathrm{u})$ underestimates $\Phi_{\text {exp }}(\mathrm{u})$ near the peak values at $\mathrm{u} \approx 2$, and overestimates them at larger values of $\mathrm{u}$. Nevertheless, we believe, given the approximations and assumptions, that the data on $\mathrm{H}$ $\mathrm{D}$ creep of $\mathrm{Al}$ can indeed be described using the universal scaling functions $\Phi(\mathrm{u})$ and $\vartheta(u)$ during the primary and steady-state creep regimes.

\section{d. Strain Rate}

The incremental strain generated by one dislocation link sweeping an area $\delta A$ is equal to 


$$
\delta \varepsilon=\frac{\alpha \cdot b \cdot \delta A}{V}
$$

where $\alpha \approx 0.5$ is geometrical constant, $\mathrm{b}$ is Burgers vector and $V$ is crystal volume. To obtain strain rate it is then necessary to sum up such contributions from all links in the distribution, per unit time, which gives

$$
\dot{\varepsilon}=\alpha b \int_{0}^{\infty} \phi(L, t) \frac{d A}{d t} d L
$$

Assuming that under stress, $\sigma$, all links bow out to the same radius $r$

$$
r=\frac{2 \Gamma}{\sigma b}
$$

where $\Gamma$ is line tension, the area $A$ under the $\operatorname{arc}$ of length $\mathrm{L}$ is equal to

$$
A=\frac{r}{2}\left(L-r \sin \frac{L}{r}\right)
$$

which differentiated with respect to time leads to

$$
\frac{d A}{d t}=\frac{r}{2}\left(1-\cos \frac{L}{r}\right) g(L, t)=r \cdot g(L, t) \sin ^{2} \frac{L}{2 r}
$$

Substituting result to the strain rate equation and assuming that during H-D creep there are no links greater than critical length $\mathrm{L}_{\mathrm{c}}$ produces

$$
\dot{\varepsilon}=\alpha b \int_{0}^{L_{c}} \phi(L, t) g(L, t) \sin ^{2} \frac{L}{2 r} d L
$$

$\mathrm{L}_{\mathrm{c}}$ is a critical link length for which link becomes unconstraint to glide or climb and it is equal to

$$
L_{c}=\pi r=\frac{2 \pi \Gamma}{\sigma b}
$$

Using scaling relations, eq (2.91) becomes

$$
\dot{\varepsilon}=\frac{\alpha \cdot b \cdot r \cdot k \cdot N}{L^{*}} \int_{0}^{u_{c}} \frac{u-1}{u^{m}} \Phi(u) \sin ^{2}\left(\frac{\pi u}{2 u_{c}}\right) d L
$$

where $u_{c}=\langle L\rangle / L^{*}$. Expending $\sin ^{2}\left(\pi \mathrm{u} / 2 \mathrm{u}_{\mathrm{c}}\right)$ in a power series, taking advantage of the fact that $\mathrm{u}<<\mathrm{u}_{\mathrm{c}}$ and utilizing relations (2.3), (2.57) and (2.88) gives the following final form of the strain rate equation for the H-D creep: 


$$
\dot{\varepsilon}=\frac{\pi C b^{3} D}{2 k_{b} T} \rho \sigma
$$

where $\mathrm{C}$ is a material constant equal to

$$
\mathrm{C}=\frac{\alpha}{\langle\mathrm{u}\rangle^{2}} \int_{0}^{\mathrm{u}_{\mathrm{c}}} \mathrm{u}^{\left.2-\mathrm{m}_{(\mathrm{u}}-1\right) \Phi(\mathrm{u}) \mathrm{du}}
$$

To test the predictive capabilities of equation (2.94) can be rewritten in a normalized form

$$
\frac{2 k_{b} T \dot{\varepsilon}}{\pi C \Omega \rho D G}=\frac{\sigma}{G}
$$

and compared with the relevant experimental data from the references listed in Table 1. Such comparison with the value of $\mathrm{C}=0.267$ obtained for aluminum (Przystupa et al.

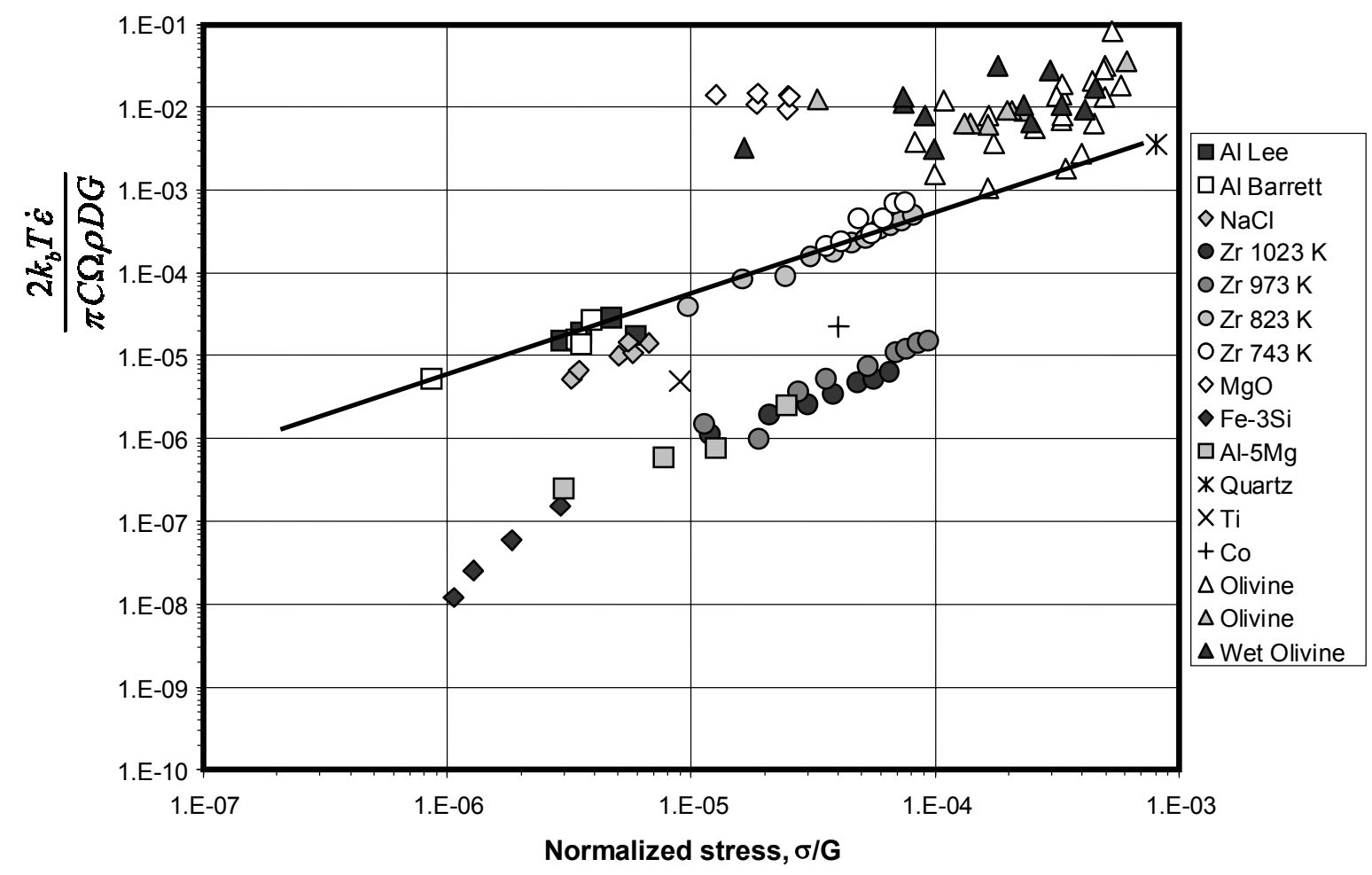

Figure 11. Comparison of the predictions from the dislocation network theory of the H-D creep, eq. (2.96), represented by the straight line with the experimental data for H-D creep from sources listed in Table 1. 
2002) and assumed the same for all materials is shown in Figure 11. Comparing Figure 11 with the similar plots for the other H-D theories reviewed earlier in this report, it is evident that the predictions of the network theory are as good and some instances even better. The two factors that definitely contributed significantly to the observed deviations from the line of perfect agreement were (1) absence of reliable data on dislocation densities for most of the materials and (2) uncertainty regarding the magnitude of the constant $\mathrm{C}$ which is most definitely not the same for all materials. Under those circumstances the obtained agreement should be considered as very good.

We also note that by combining equation (2.94) with (2.68) and (2.57) one obtains the following relation for the strain rate:

$$
\dot{\varepsilon}=\frac{\beta^{2} \kappa C b^{2}}{16 \Gamma\langle u\rangle} \frac{(m+1)^{m+1}}{m^{m}} \rho \sigma
$$

Since values of $\kappa, \mathrm{m}, \mathrm{C}$ and $\langle\mathrm{u}\rangle$ can be obtained from the static annealing data on the dislocation annealing kinetics and link length distributions, eq. (2.97) can be instrumental in the verifications of the predictive capabilities of the dislocation network theory of H-D creep. 


\section{Experimental Procedures and Results}

The emphasis of the experimental part of the program was on quantitative characterization of the evolution of the dislocation networks during annealing of pure aluminum, zinc and tin samples, representing materials with fcc, hcp and bcc crystal structure, respectively. For this purpose the aluminum samples were pre-deformed by creep at 913 and $619 \mathrm{~K}$ and then annealed. The higher deformation temperature was selected to generate starting dislocation networks similar to those forming during HarperDorn creep and the lower, to obtain higher dislocation densities suitable for reliable estimates of the parameters of the network growth law. The annealing temperatures for samples pre-deformed at 913 were 913,863 and $817 \mathrm{~K}$ and for those deformed at $619 \mathrm{~K}$ they were 913,733 and $619 \mathrm{~K}$. The particular temperatures were selected to obtain data points equally spaced on the $1 / \mathrm{T}$ scale, which is convenient for further analysis. In case of zinc the samples were pre-deformed by creep to steady state region at $573 \mathrm{~K}$ and subsequently annealed at the same temperature for various times. The testing temperature was selected to assure operation of the same dislocation network coarsening mechanisms during annealing as those expected during H-D creep with higher dislocation density suitable for reliable characterization of the dislocation structure. For tin the creep and annealing temperature was $423 \mathrm{~K}$, chosen again as for zinc to assure operation of the same network recovery and refinement mechanism during annealing and H-D at higher dislocation densities. After annealing, the samples were polished and used for measurements of the dislocation densities and dislocation link-length distributions using etch-pit methods. The obtained results were subsequently used for the verification of the predictions from the dislocation network theory of the H-D cree. The results obtained and specifics of the experimental procedures used, are described in more detail in the following sections.

\section{Characterization of Dislocation Structures}

All characterizations of the dislocation structures were done using the etch pit method. The reason for selecting that method was that it was the only method that allowed for the measurements of the low dislocation densities expected during H-D creep 
and annealing. We also have significant experience using that method, particularly for aluminum (Ardell et al. 1986, Lin et al, 1985). The obtained etch-pit dislocation structures were scanned and the resulting images used as input to the Image-Pro quantitative image analysis program. The dislocation densities were be subsequently estimated using equation(Ham et al, 1961):

$$
\rho=\frac{2 \mathrm{~N}}{\mathrm{~A}}
$$

where $\mathrm{N}$ is the number of etch pits not associated with subgrain or grain boundaries and A is the corresponding test area. The distribution of dislocation link lengths was then obtained from the coordinates of the etch pits using the method developed by us in our previous etch pit studies (Lin et al., 1985 and 1989). This method is an adaptation of the TEM method used for the measurement of link lengths in thin foils by Oden et al.1974. It has been modified by us for the use with the etch pit results and successfully tested in the characterizations of the link length distributions in our earlier studies on the creep behavior of $\mathrm{Al}$ and $\mathrm{NaCl}$ (Lee, 1985, Lin et al., 1989). It assumes that link lengths, $\ell_{\mathrm{i}}$, are related to the local dislocation densities, $\rho_{\mathrm{i}}$, through relation $\ell \approx \rho_{\mathrm{i}}^{-1 / 2}$. The $\rho_{\mathrm{i}}$ are then estimated from the distances between projections of the etch pits on an arbitrary test line.

We have also investigated the possibility of using other alternative methods of finding local dislocation densities which could give more precise estimates of the linklengths. The first of those methods, shown schematically in the upper part of Figure 12, employs Voronoi diagrams. In this method the dislocation pit structure is divided into Voronoi polygonal cells with each polygon encompassing the area that is closer to a given pit than to any other pit. The reciprocals of the cell areas represent the local dislocation densities which are then used to obtain link lengths. As shown in the figure, our current method gives a larger number of links in the small size range compared to that obtained from Voronoi diagrams. Similar result has been obtained from the second new method which is based on representing dislocation network with uniform 3connected nets. This method is shown schematically in the lower part of Figure 12. It assumes that links form three-dimensional network build from basic units shown in the 


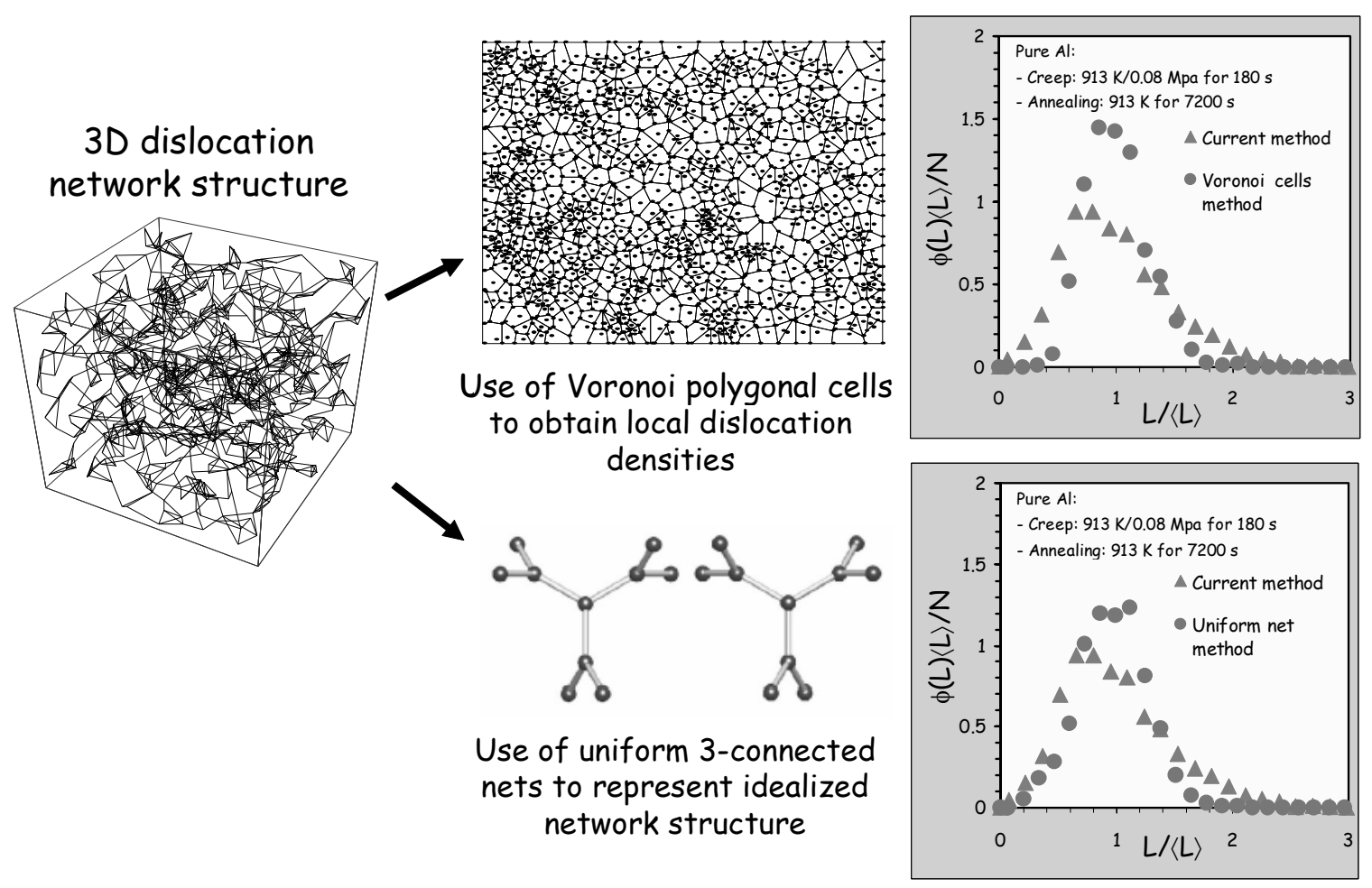

Figure 12. Schematic showing procedures for refining current method for obtaining linklength distributions by utilizing Voronoi cells and 3-connected uniform nets.

middle part of the figure (Delgado et al, 2003). Such network consists of regular n-gons which can be approximated by circles. Assuming that etch pit structure represents plane section through the network of circles allows finding distribution of circle diameters using standard Saltykov method (Undrwood, 1970). The link lengths can be than obtained from circle diameters through rudimentary algebra. Results of such calculations, assuming the statistically most probable network of 10-gons, are shown in Figure 12. As for the Voronoi diagrams, this method also gives smaller number of links in the initial part of the distribution compared to our current method. This difference is significant because that particular part of the distribution is used to estimate exponent $m$ in our link growth law. Both methods require further testing before they can be used in confidence in the analysis of the etch pit results. 


\section{Network Evolution Studies}

\section{a. FCC Aluminum}

The aluminum samples for annealing studies were prepared from $99.999 \% \mathrm{Al}$ pellets purchased from the Kurt J. Lesker Company, Clarington, PA. The pellets were melted in a vertical furnace in a graphite crucible under an Ar atmosphere. The defectfree ingots were obtained by following a processing sequence consisting of initial melting for $23 \mathrm{~min}$ at $1050 \mathrm{~K}$ followed by cool-down and soaking for $17 \mathrm{~min}$ at $950 \mathrm{~K}$ then for 20 $\mathrm{min}$ at $930 \mathrm{~K}$ and finally slow cooling to room temperature. The final ingots had a diameter of $23 \mathrm{~mm}$ and were $65 \mathrm{~mm}$ long. They were cut with a diamond saw to parallelepiped-shaped creep samples. To remove surface damage introduced during machining all the samples were chemically polished by immersion for $15 \mathrm{~min}$ at $373 \mathrm{~K}$ in a polishing solution consisting of $60 \% \mathrm{H}_{2} \mathrm{SO}_{4}+30 \% \mathrm{H}_{3} \mathrm{PO}_{4}+10 \% \mathrm{HNO}_{3}$. After polishing the samples were annealed for 72 hour at $913 \mathrm{~K}$, which resulted in a final dislocation density of about $800 \mathrm{~mm}^{-2}$. We attempted to use dilute $\mathrm{Al}+1.0$ at. $\% \mathrm{Mg}$ and $\mathrm{Al}+0.05$ at. $\% \mathrm{Cu}$ alloys to obtain lower dislocation densities. The solutes present in those alloys should pin dislocations and prevent their multiplication due to thermal stresses and/or handling. After annealing both alloys for $72 \mathrm{hrs}$ at $640 \mathrm{~K}$, the lowest dislocation density in the $\mathrm{Al}-\mathrm{Cu}$ alloy was $690 \mathrm{~mm}^{-2}$ and in the $\mathrm{Al}-\mathrm{Mg}$ alloy it was in the $4000 \mathrm{~mm}^{-2}$ range. An alternative two-step annealing of the same alloys for $72 \mathrm{hrs}$ at $913 \mathrm{~K}$, followed by $72 \mathrm{hrs}$ at $926 \mathrm{~K}$, did not result in any significant reduction of the final dislocation density. Since the dislocation densities in the solid solution alloys were either larger or not very different from those in pure aluminum, the alloys were excluded from further studies.

After annealing, the samples were crept in argon atmosphere in compression at $913 \mathrm{~K}$ and $619 \mathrm{~K}$ under constant stress and then cooled under load to preserve the high temperature dislocation network structure. The loading was done using a custom built creep apparatus with a radiation furnace and a lever-type constant-load loading system. The advantage of using the radiation furnace is that it allows for rapid heating and cooling, which facilitates preservation of both the initial and the as-loaded dislocation structures. The furnace has a temperature stability of $\pm 1 \mathrm{~K}$ and a cooling rate of $5.5 \mathrm{~K} / \mathrm{s}$, 
which is more than adequate for our purposes. The deformed samples were subsequently sliced into $6 \mathrm{~mm}$ thick disks and annealed also in argon atmosphere. To obtain the dislocation etch pit structure the samples had to be first mechanically polished, then electropolished and finally etched. The preliminary mechanical polishing was done using, in sequence, 320, 600, 800 and 120 mesh paper. For the final fine polishing steps 1 and $0.05 \mu \mathrm{m}$ alumina powder were used. To remove possible surface damage resulting from mechanical polishing the samples were electropolished in the solution of $15 \% \mathrm{HClO}_{4}$ in methanol cooled to $-60^{\circ} \mathrm{C}$ by adding liquid nitrogen. The electropolishing took 45 minutes to 1 hour at $12 \mathrm{~V}$ and $150 \mathrm{~mA}$. This particular procedure was used by us in our previous work on $\mathrm{Al}$ and showed to be effective in obtaining accurate and reproducible results (Lee, 1985). The final etch pitting consisted of etching the surface with the mixture of $50 \% \mathrm{HCl}, 47 \%+\mathrm{HNO}_{3}+3 \% \mathrm{HF}$ at $-35{ }^{\circ} \mathrm{C}$ for 35 to 45 seconds. As seen in Figure 13 the etch pits obtained were always well defined, clearly revealing the dislocation structure without sacrificing surface brightness. The etch pit densities were measured using an optical microscope at a magnification of $200 \mathrm{x}$. The reported dislocation densities were obtained based on an average etch-pit density taken from at least ten different areas.

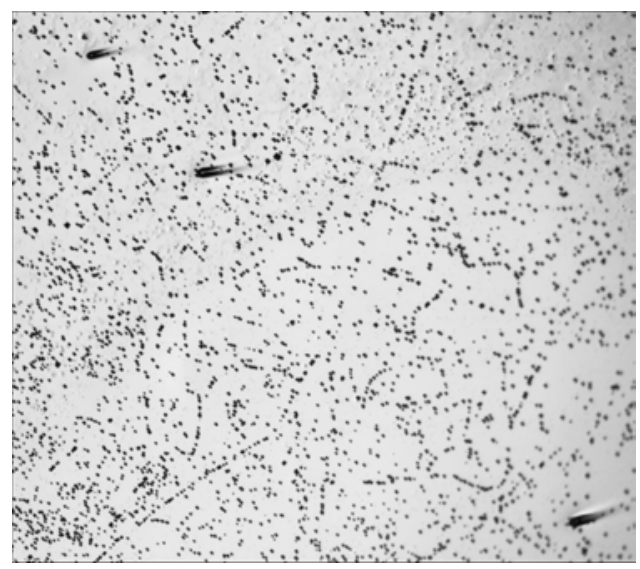

(a)

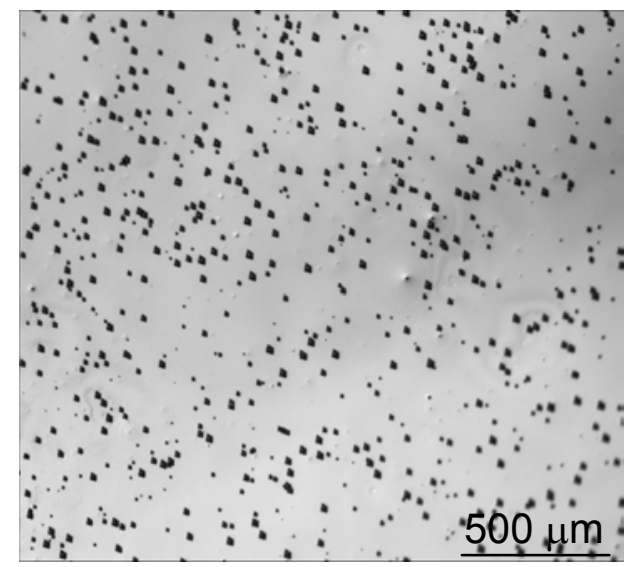

(b)

Figure 13. Dislocation structures in pure aluminum samples loaded in compression for $180 \mathrm{~s}$ at a constant stress of $0.08 \mathrm{MPa}$ at $913 \mathrm{~K}$ and then annealed for (a) $300 \mathrm{~s}$ $\left(\rho=1029 \mathrm{~mm}^{-2}\right)$ and (b) $7200 \mathrm{~s}\left(\rho=389 \mathrm{~mm}^{-2}\right)$ at the same temperature. 


\section{i. Samples pr-deformed at $913 \mathrm{~K}$}

All samples pre-deformed at $913 \mathrm{~K}$ had dimensions 9x9x20 mm and were loaded in compression for $180 \mathrm{~s}$ at a constant stress of $0.08 \mathrm{MPa}$ and than annealed at at 913, 863 and $817 \mathrm{~K}$ for times ranging from $300 \mathrm{~s}$ to $2 \mathrm{hrs}$. Figure 13 shows typical dislocation etch pit structure for samples annealed after loading at $913 \mathrm{~K}$. For short annealing times there is a tendency to form faint low-angle grain boundaries (Figure 13a). Those boundaries dissolve during further annealing and the dislocation structure becomes uniform Figure 13b). The changes of the dislocation densities during annealing for different temperatures are shown in Figure 14. The plateau density appears to be the same for all three annealing temperatures and equal to $323 \pm 60.4 \mathrm{~mm}^{-2}$. This is the "frustration density", that is the density at which the availability of the links with Burgers vectors suitable for the annihilation reactions has been exhausted. It is important to point out that

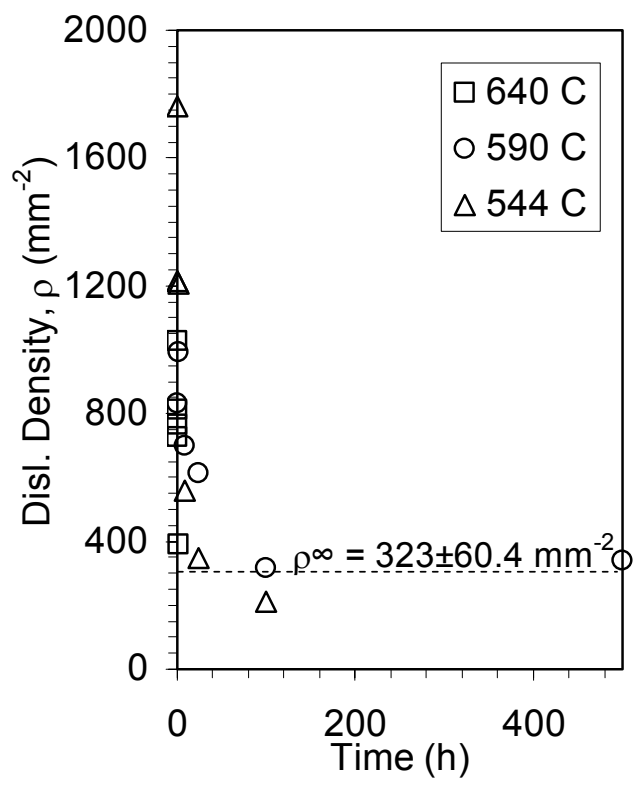

(a)

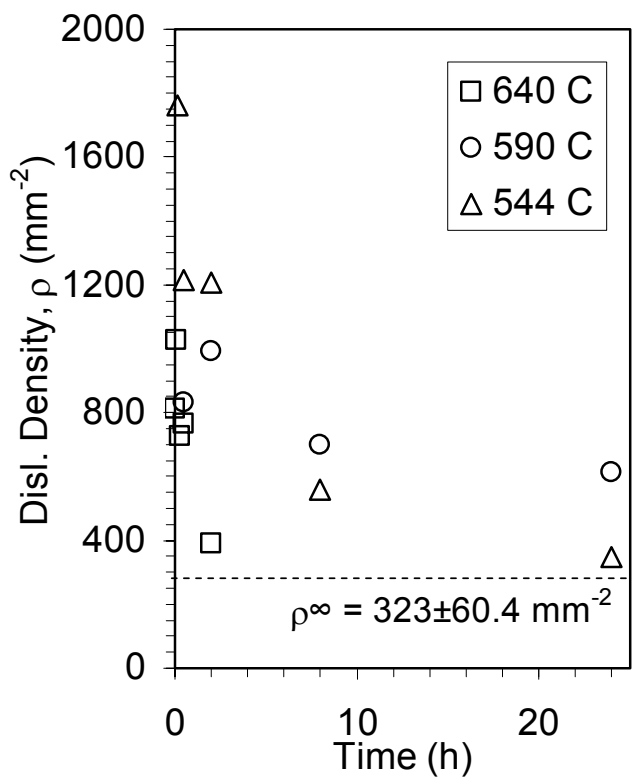

(b)

Figure 14. Change of dislocation density with time in pure Al samples during annealing at the indicated temperatures for (a) long and (b) short times. Prior to annealing the samples were crept at $913 \mathrm{~K}$ for $180 \mathrm{~s}$ under a constant stress of $0.08 \mathrm{MPa}$. The line shows the average plateau dislocation density level. 


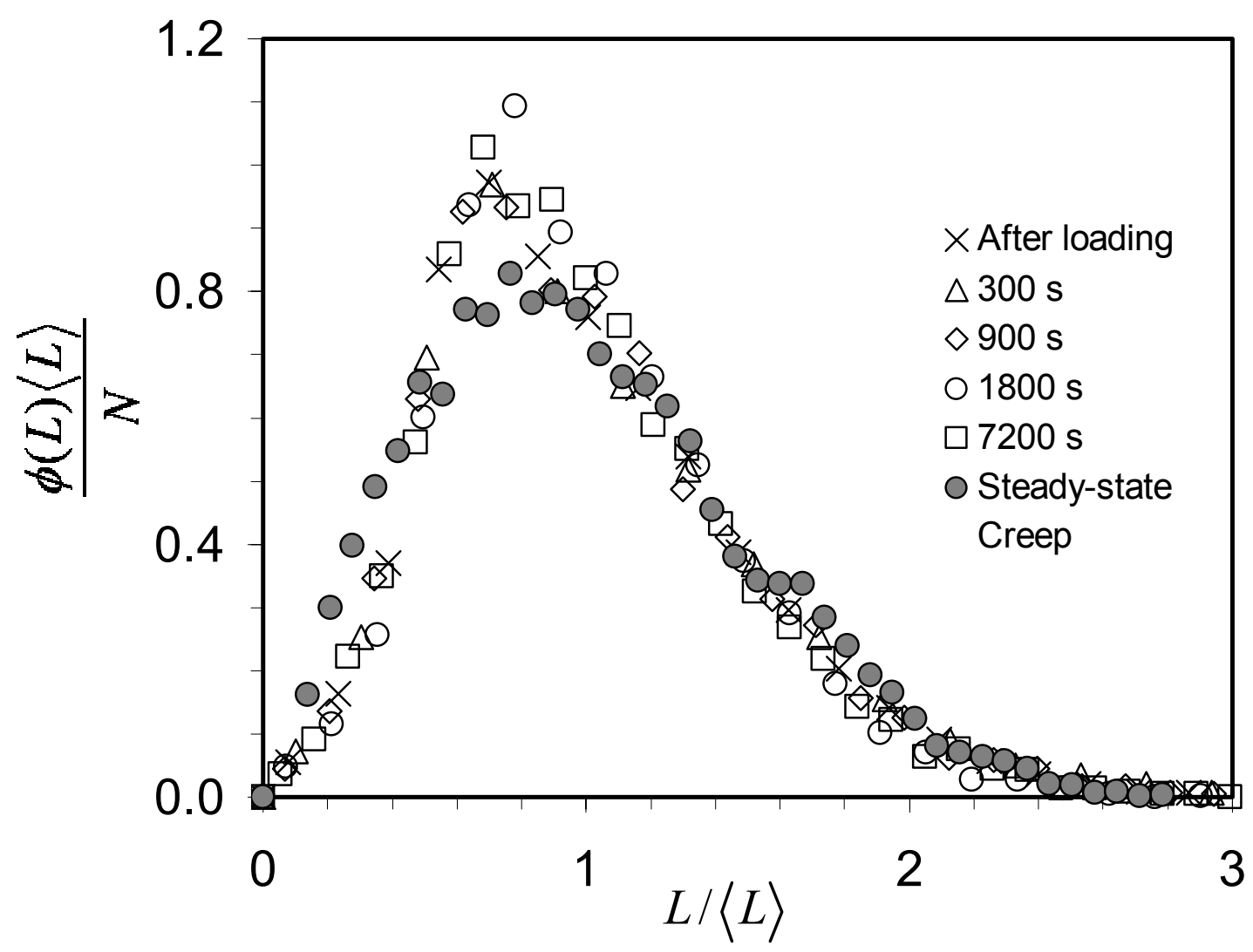

Figure 15. Experimental results showing the normalized dislocation link length distributions for pure aluminum samples after loading at $913 \mathrm{~K}$ in compression at constant stress of $0.08 \mathrm{MPa}$ for $180 \mathrm{~s}$ and subsequently unloaded and statically annealed at the same temperature for the indicated times. Dark circles are for the distribution obtained after Harper-Dorn creep at the same constant stress and temperature to steady-state.

for annealing at $913 \mathrm{~K}$ the link exhaustion time is very short shorter. This means that obtaining reliable data on the dislocation density kinetics to determine the slope of the initial linear part of the curve at that temperature is imply impossible. Figure 15 shows a comparison of the initial and annealed dislocation link-length distributions with the linklength distribution for aluminum obtained under creep conditions in our earlier studies of Harper-Dorn creep (Lee 1985). The data from all the distributions collapse onto a single curve, which is in accord with our theoretically predicted time-invariant scaling behavior. 
This result is the first ever convincing proof that the evolution of the dislocation network during static annealing is the same as that during Harper-Dorn creep. Figure 16 shows a fit of the function $\phi(L)=C \cdot L^{m}$ lines to the initial portions of the distributions. The average slopes of the lines provide the experimental values of the exponent, $m$, in our link growth law, $g(L, t)$ The average value of $m$ is $1.291 \pm 0.07$, which is in excellent agreement with the value of $m=1.33$ obtained during Harper-Dorn creep [3]. This is additional evidence that the kinetics of static annealing and coarsening of the dislocation networks during Harper-Dorn creep can be described by the same growth law.

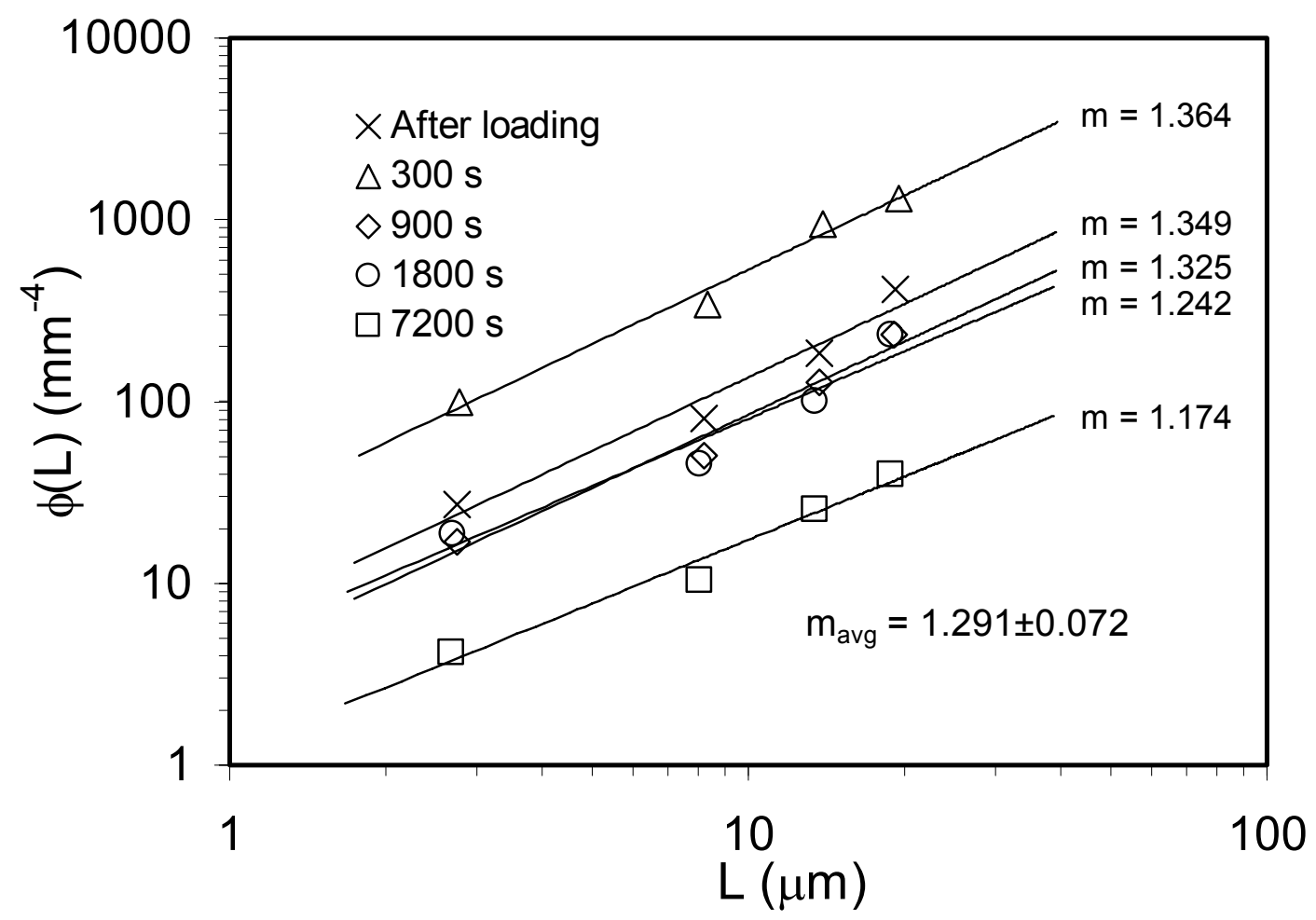

Figure 16. Experimental results showing a fit to the initial portion of the distributions shown in Figure 15 to obtain the exponent $\mathrm{m}$ in the link growth law. All results are for pure aluminum samples after loading at $913 \mathrm{~K}$ in compression at constant stress of $0.08 \mathrm{MPa}$ for $180 \mathrm{~s}$ and subsequently unloaded and statically annealed at the same temperature for the indicated times. 


\section{ii. Samples pr-deformed at $620 \mathrm{~K}$}

All the samples used in the creep and annealing studies at $620 \mathrm{~K}$ were prepared using the same procedure as the one described in the proceeding section. The final creep samples had dimensions $7 \mathrm{~mm} \times 7 \mathrm{~mm} \times 20 \mathrm{~mm}$. After cutting and polishing the samples were annealed for 1 hour at $723 \mathrm{~K}$ Subsequent creep tests were designed to (1) determine the time to reach steady-state, hence the time necessary to develop a stable dislocation network structure and (2) to find the relationship between the steady-state dislocation density and the applied stress. All the creep tests were conducted in a radiant furnace at $619 \mathrm{~K}$ under constant load and initial stresses of 2, 3.34 and $5 \mathrm{MPa}$. The resulting creep curves are shown in Figure 17a. The correlation between the final steady-state dislocation densities and stress is shown in Figure 17b. The trend line shown in the figure represents best fit of the data to the standard relation:

$$
\sigma=\sigma_{o}+\alpha M G b \sqrt{\rho}
$$

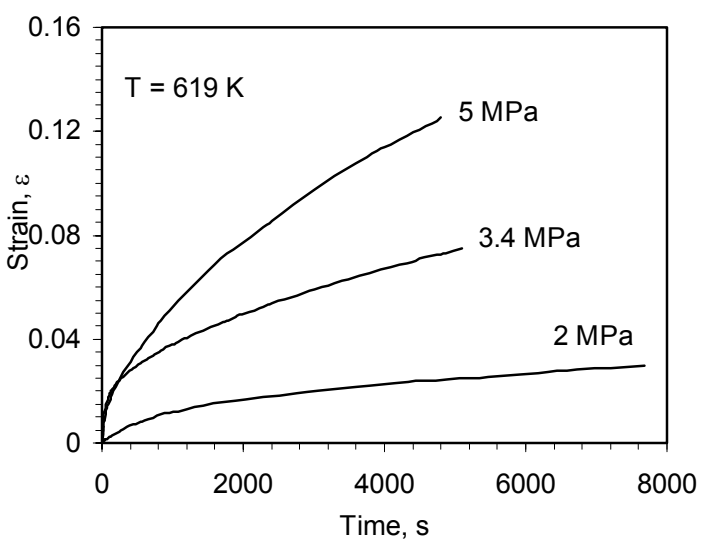

(a)

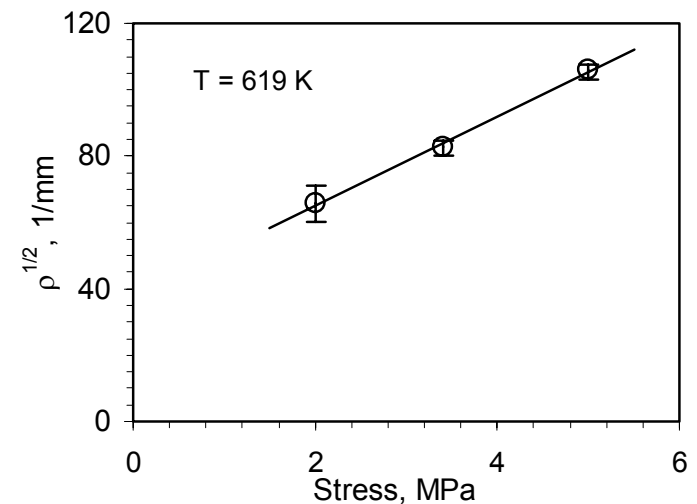

(b)

Figure 17. Results of the creep tests conducted for Al samples at $619 \mathrm{~K}$ under constantstress conditions: (a) creep curves and (b) change of steady-state dislocation density with stress.

where $\sigma_{o}$ and $\alpha$ are fitting constants, $M$ is the Taylor factor, $G$ the shear modulus and $b$ the Burgers vector. The best fit was obtained with $\sigma_{o}=-2.83 \mathrm{MPa}$ and $\alpha M G b=0.074$ MPa $\cdot \mathrm{mm}$. Assuming $M=3.06$, which is appropriate for our case of a random polycrystal, 
and $\mathrm{G}$ and $\mathrm{b}$ for pure aluminum gives $\alpha=3.77$. Our values of $\sigma_{o}$ and $\alpha$ are slightly higher than those obtained under similar testing conditions for aluminum single crystals by Daily and Ahlquist, 1972. The difference can probably be attributed to the differences in material purity and the effects of grain boundaries.

After interrupted creep tests the samples were annealed for times ranging from 10 $\mathrm{s}$ to 64 hours at temperatures of 619,733 and $913 \mathrm{~K}$ to determine the kinetics of the variation of the dislocation density with annealing time. The obtain data were critical for reliable estimates of the phenomenological constants in the equation describing annealing

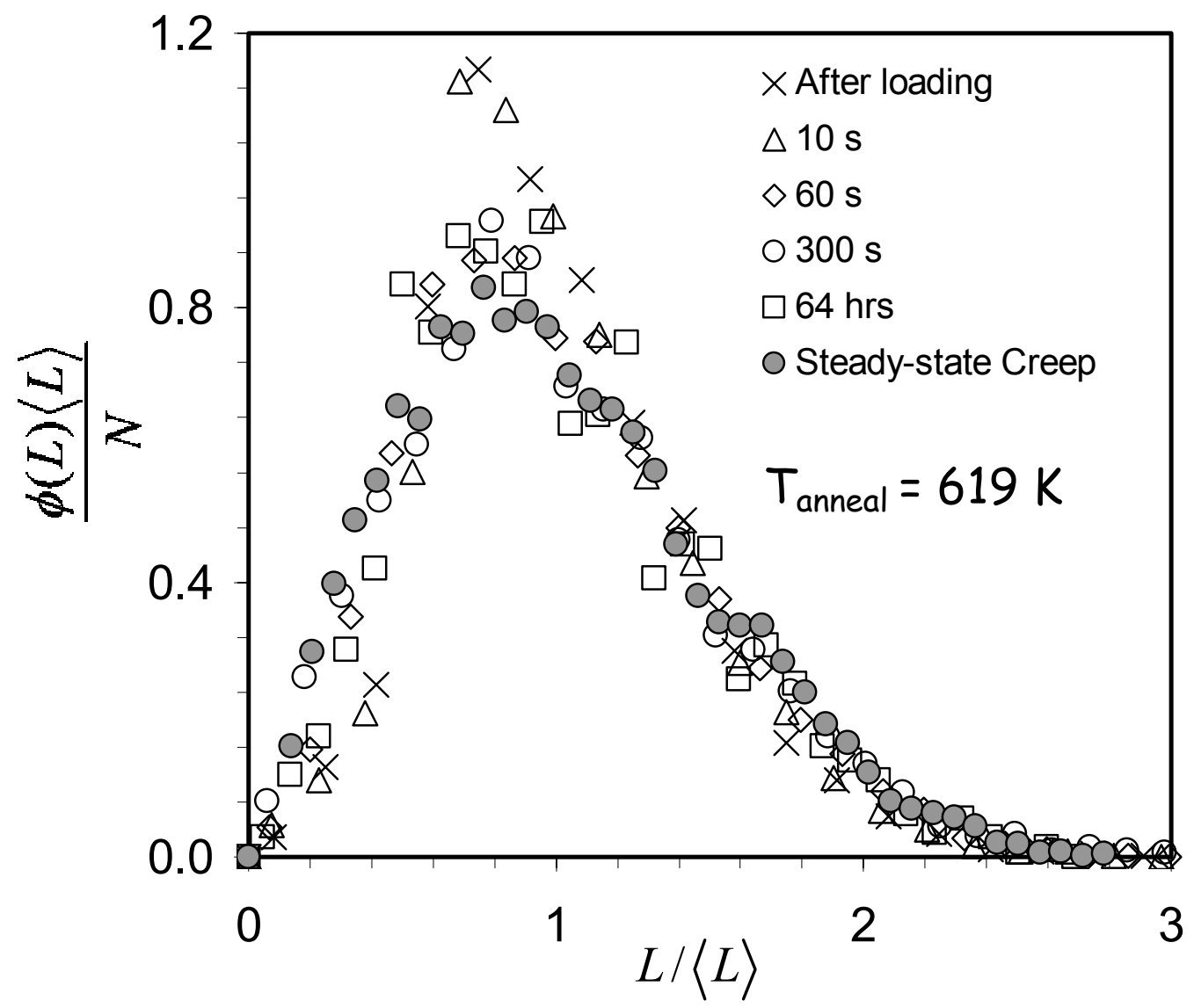

Figure 18. Experimental results showing the normalized dislocation link length distributions for pure aluminum samples after creep at $619 \mathrm{~K}$ in compression at constant stress of $5 \mathrm{MPa}$ to steady-state and subsequently unloaded and statically annealed at the same temperature for the indicated times. Dark circles are for the distribution obtained after Harper-Dorn creep at temperature $913 \mathrm{~K}$ the constant stress of $0.08 \mathrm{MPa}$ to steady-state. 


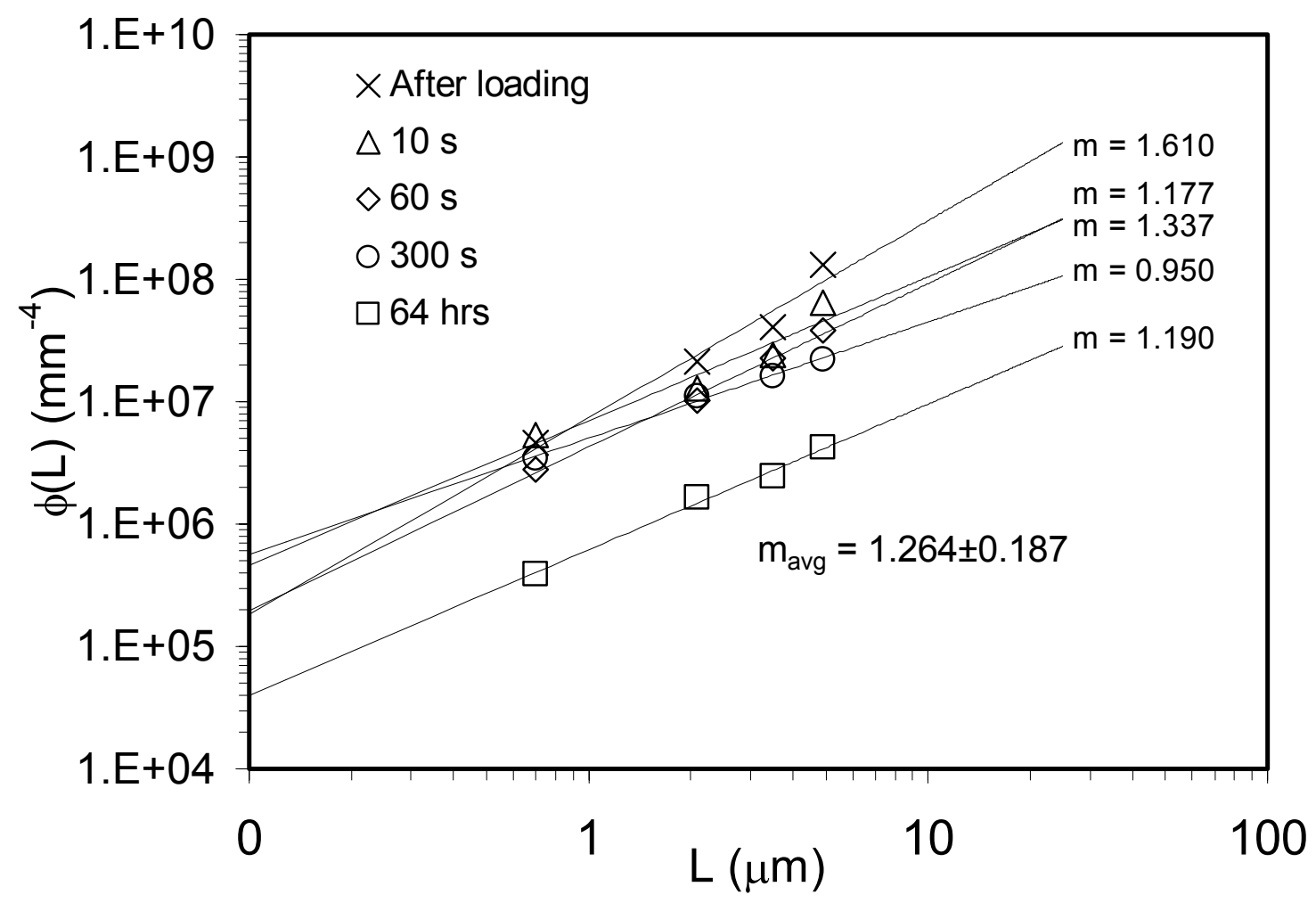

Figure 19. Experimental results showing a fit to the initial portion of the distributions shown in Figure 18 to obtain the exponent $m$ in the link growth law. All results are for pure aluminum samples after creep at $619 \mathrm{~K}$ in compression at constant stress of $5 \mathrm{MPa}$ to steady state and subsequently unloaded and statically annealed at the same temperature for the indicated times.

kinetics. To obtain the values of those constants it was necessary to (1) perform annealing at two different temperatures and (2) establish that the scaled link-length distributions in both temperatures were also time invariant and identical to those obtained during H-D creep. The temperature chosen for estimating rate constant were $733 \mathrm{~K}$ and the creep test temperature of 619 K. Figure 18 shows scaled dislocation link-length distributions for annealing at $619 \mathrm{~K}$. The distributions for all annealing times superimpose and are similar to that for H-D creep which is also shown in the figure; similar behavior was also observed for the other annealing temperature as shown in Figure 20 and Figure 21. This 
is the first ever results supporting our theoretical predictions that the scaled dislocation link length distributions are time invariant and are the same for annealing and H-D creep Figure 19 shows initial portions of the distributions which were used to estimate exponent $m$ in our link growth law discussed earlier in the report. The average initial slope of the distributions was $1.264 \pm 0.187$ which compares very favorably with the value of $\mathrm{m}=1.33$ obtained for H-D creep.

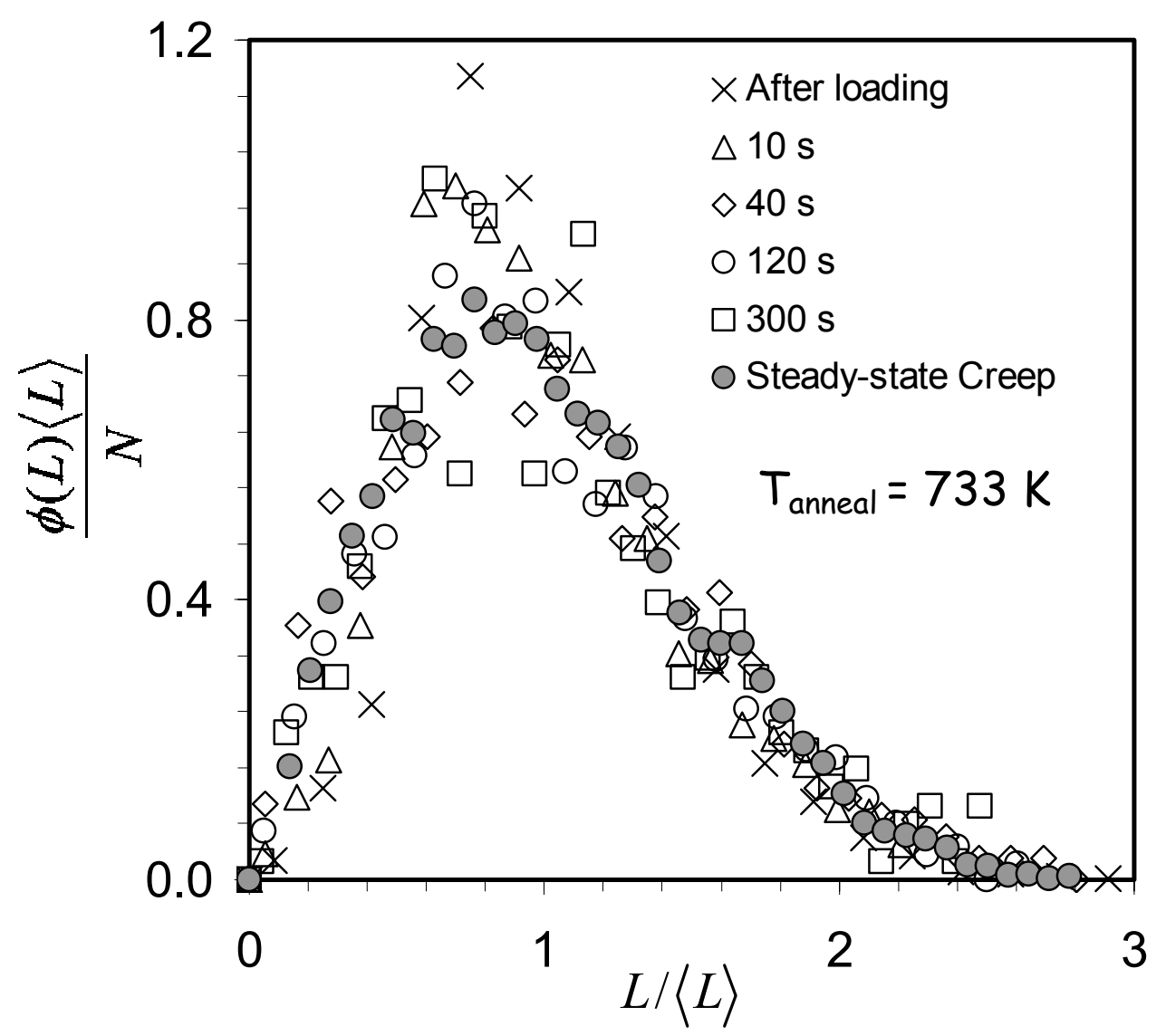

Figure 20. Experimental results showing the normalized dislocation link length distributions for pure aluminum samples after creep at $619 \mathrm{~K}$ in compression at constant stress of $5 \mathrm{MPa}$ to steady-state and subsequently unloaded and statically annealed at $733 \mathrm{~K}$ for the indicated times. Dark circles are for the distribution obtained after Harper-Dorn creep at temperature $913 \mathrm{~K}$ the constant stress of $0.08 \mathrm{MPa}$ to steady-state. Average initial slope of the distribution, $\mathrm{m}$, is $1.156 \pm 0.313$ 


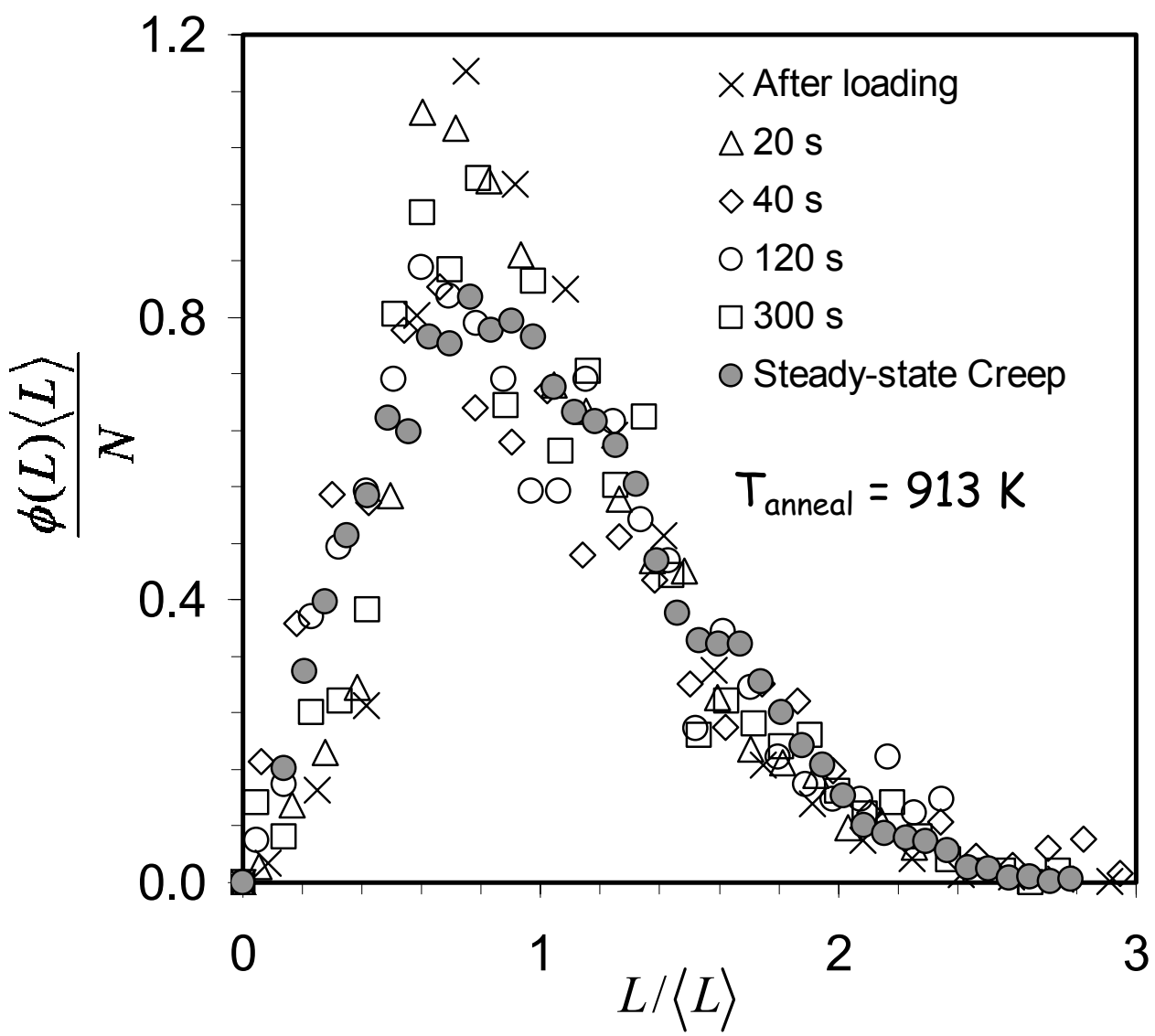

Figure 21. Experimental results showing the normalized dislocation link length distributions for pure aluminum samples after creep at $619 \mathrm{~K}$ in compression at constant stress of $5 \mathrm{MPa}$ to steady-state and subsequently unloaded and statically annealed at $913 \mathrm{~K}$ for the indicated times. Dark circles are for the distribution obtained after Harper-Dorn creep at temperature $913 \mathrm{~K}$ the constant stress of $0.08 \mathrm{MPa}$ to steady-state. Average initial slope of the distribution, $\mathrm{m}$, is $1.067 \pm 0.405$

Figure 22a shows the change of dislocation density with annealing time for samples annealed at $619 \mathrm{~K}$ after deforming them, at the same temperature and at the indicated stresses, up to steady state. As expected the dislocation density $\rho$ decreases rapidly with annealing time and reaches the frustration/plateau level in less than a minute. The frustration density is independent of the initial dislocation density, and varies from 3500 


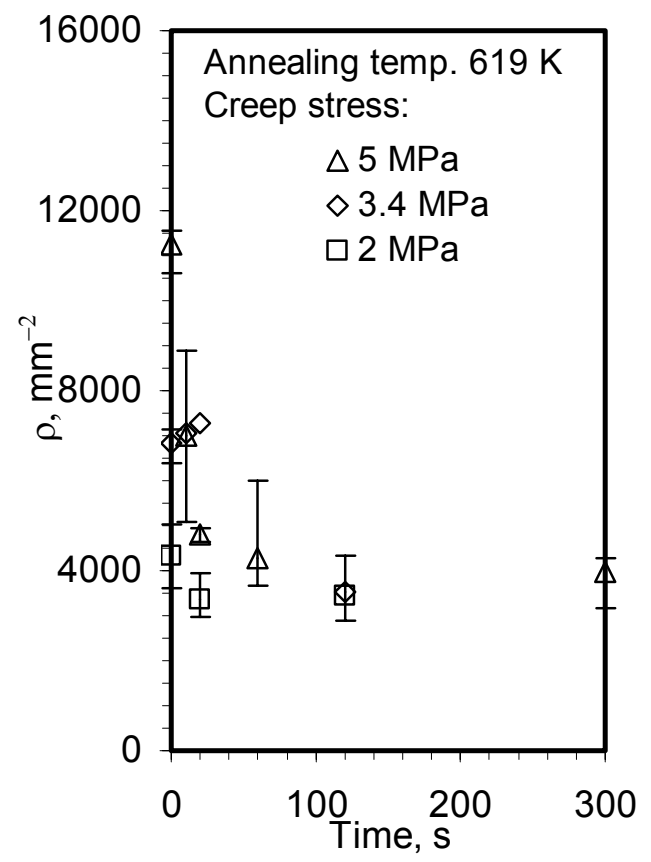

(a)

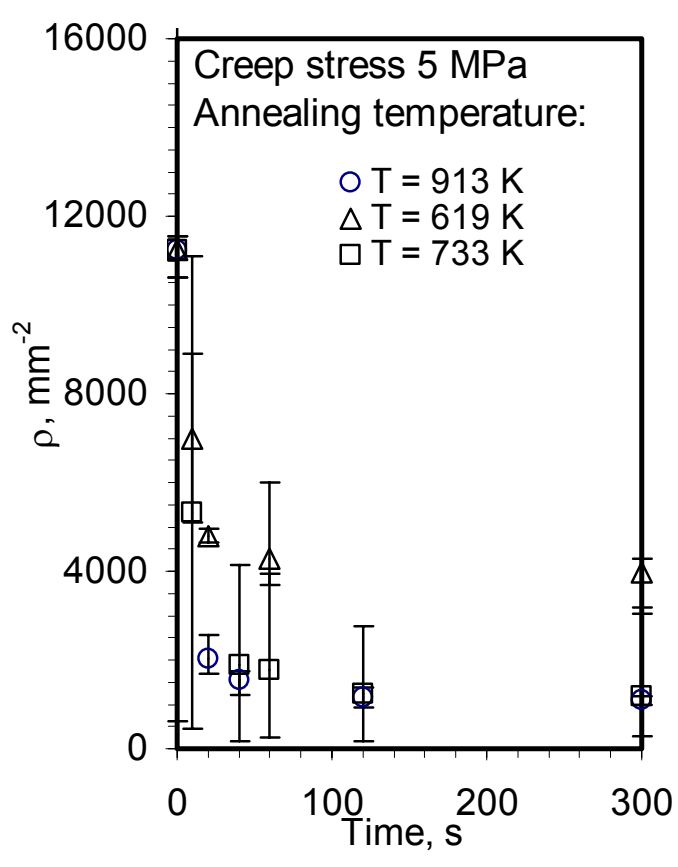

(b)

Figure 22. Change of dislocation density for aluminum with annealing time for (a) samples crept to steady-state at indicated stresses and then annealed at $619 \mathrm{~K}$ and (b) samples crept to steady-state at $5 \mathrm{MPa}$ and then annealed at indicated temperatures. In both cases the creep was conducted at $619 \mathrm{~K}$.

to $4000 \mathrm{~mm}^{-2}$. However, the frustration level does depend on the annealing temperature, as shown in Figure 22b and Figure 23. These figures compare changes of $\rho$ with time for identical initial dislocation networks annealed at different temperatures. For higher annealing temperatures the change of the dislocation density is more rapid and the final frustration dislocation level is smaller $\left(\sim 1000 \mathrm{~mm}^{-2}\right)$. The results in Figure $22 \mathrm{~b}$ and Figure 23 , for annealing times up to $5 \mathrm{~min}$, are re-plotted as $1 /\left(\rho-\rho_{\infty}\right)$ vs time in Figure 24. This plot makes possible estimation of constant $\kappa(T)$ in our slightly modified dislocation density vs annealing time relation:

$$
\frac{1}{\rho-\rho_{\infty}}-\frac{1}{\rho_{o}-\rho_{\infty}}=\kappa(T) \cdot t=\frac{C_{\kappa}}{T} \exp \left(-\frac{Q}{R \cdot T}\right) \cdot t
$$




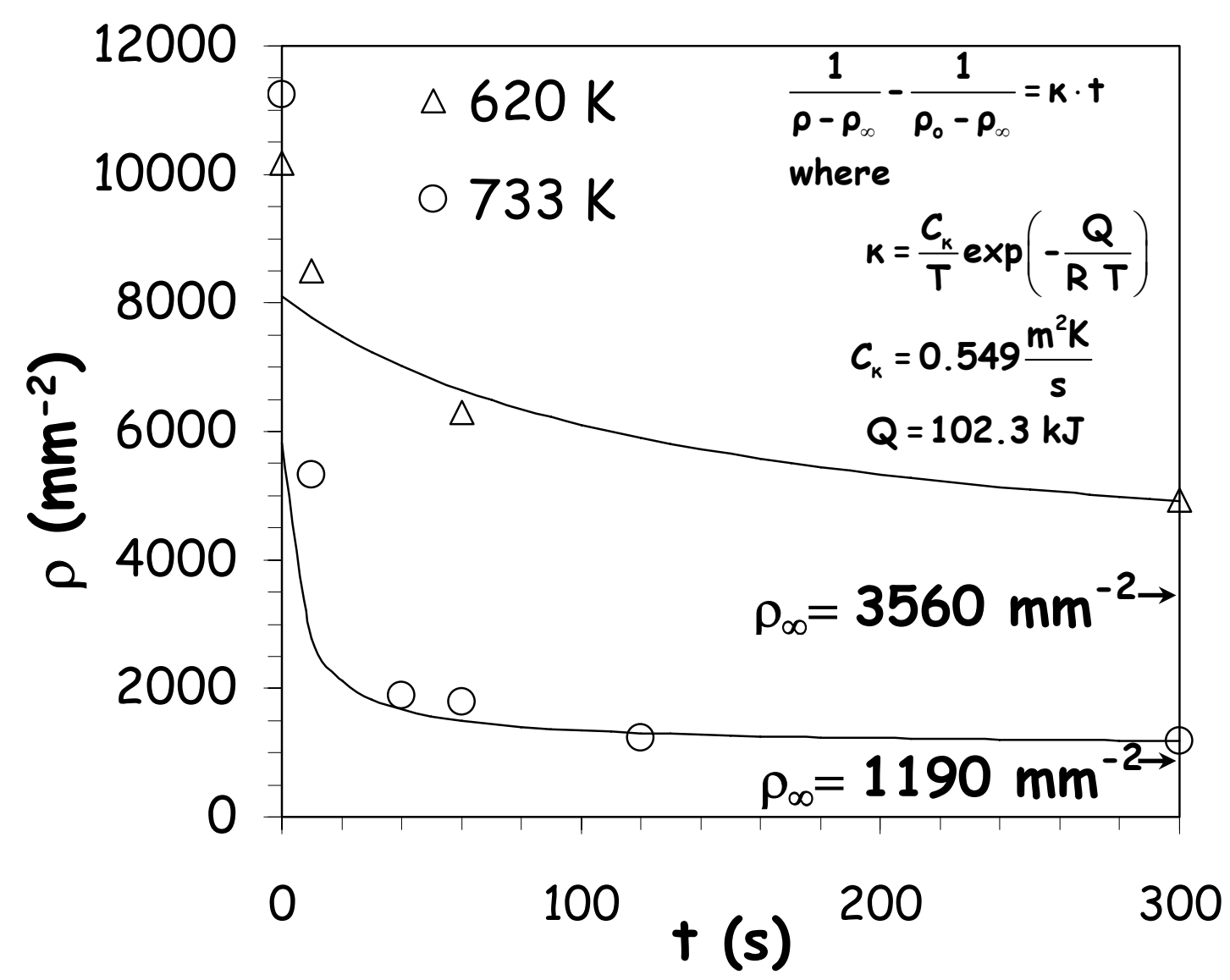

Figure 23. Results of fitting annealing data for 620 and $733 \mathrm{~K}$ from Figure $22 \mathrm{~b}$ to eq. The values of the best-fit constants as well as the frustration dislocations densities $\mathrm{r}$ are shown in figure. Prior to annealing the samples were pre-deformed in compression to steady state at constant stress of $5 \mathrm{MPa}$ at $620 \mathrm{~K}$.

where $\rho_{\infty}$ is the "frustration" dislocation density. This relation is a modified version of eq. (2.67) and it that takes into account our assertion that during annealing and H-D creep dislocation density does not "anneal out" but rather tends asymptotically to a frustration plateau. However, equations (2.67) and (3.3) have the same slope at the limit of $t \rightarrow 0$, which means that best fit of eq. (3.3) can be used to find $\kappa$. Using frustration dislocation density $\rho_{\infty}$ of $3560 \mathrm{~mm}^{-2}$ for $620 \mathrm{~K}$ and $1190 \mathrm{~mm}^{-2}$ for $733 \mathrm{~K}$ the best fit shown in Figure 24 gives values of $\kappa$ equal to $3.86 \cdot 10^{-11} \mathrm{~m}^{2} / \mathrm{s}$ and $1.73 \cdot 10^{-12} \mathrm{~m}^{2} / \mathrm{s}$ for temperatures $733 \mathrm{~K}$ 


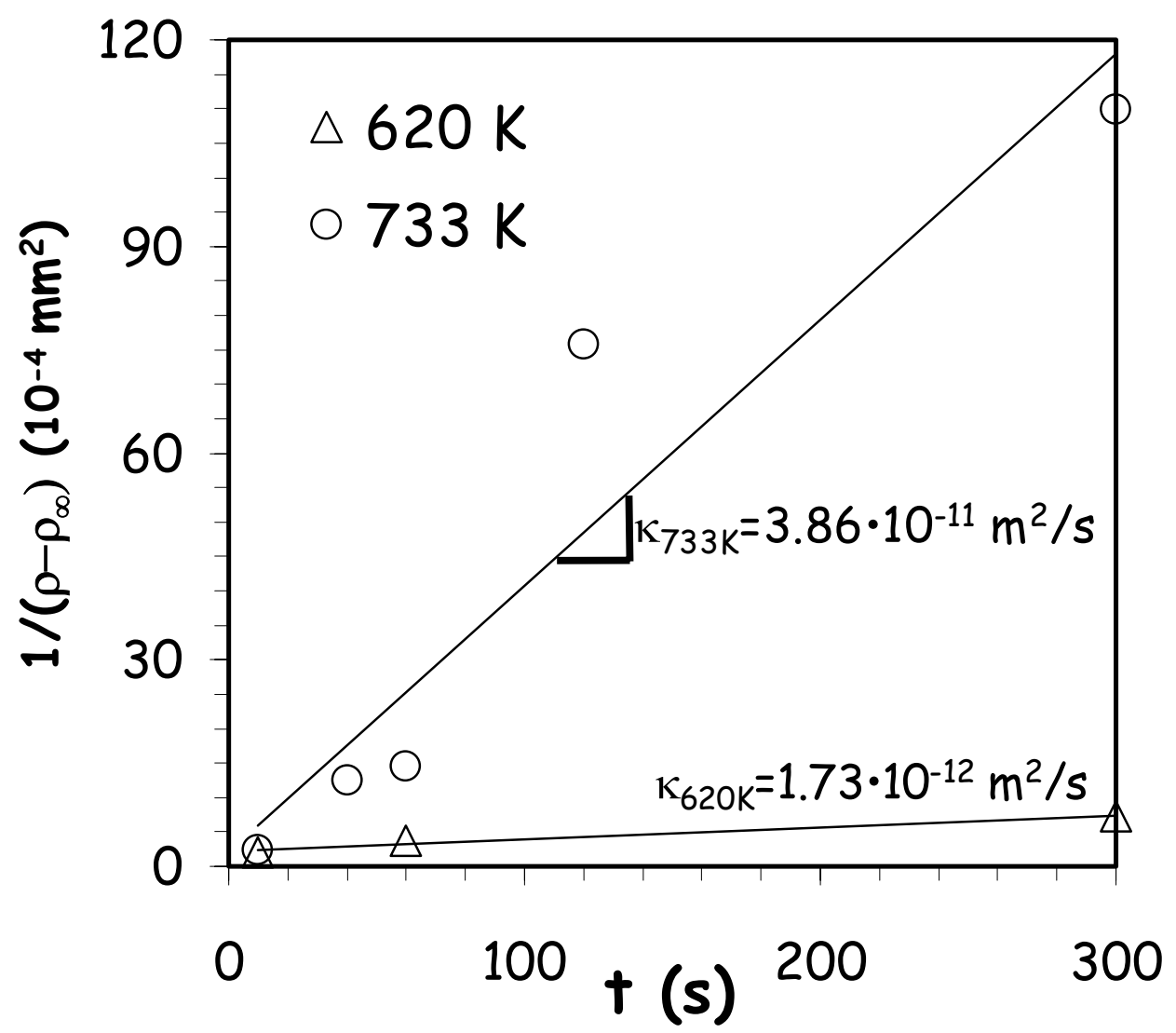

Figure 24. Plot of the reciprocal of the dislocation density vs time for samples previously crept to steady-state at $619 \mathrm{~K}$ at a constant stress of $5 \mathrm{MPa}$ and subsequently annealed at the indicated temperatures.

and $620 \mathrm{~K}$, respectively. Based of those results the estimated value of constant $\mathrm{C}_{\mathrm{K}}$ is $0.549 \mathrm{~m}^{2} \cdot \mathrm{K} / \mathrm{s}$ and the activation energy $\mathrm{Q}$ is equal to $102.3 \mathrm{~kJ}$. These results constitute a complete set of information needed to predict H-D steady-state creep rates from static annealing data using eq. (2.97). Comparison of such predictions with the experimental data for H-D creep of aluminum at $920 \mathrm{~K}$ is shown in Figure 2. The experimental rates are on average 4.54 times higher than the predicted values which should be considered as an outstanding result. It is important to point out that this is the first time ever that H-D creep rates have been predicted from the annealing data without any adjustable parameters. 


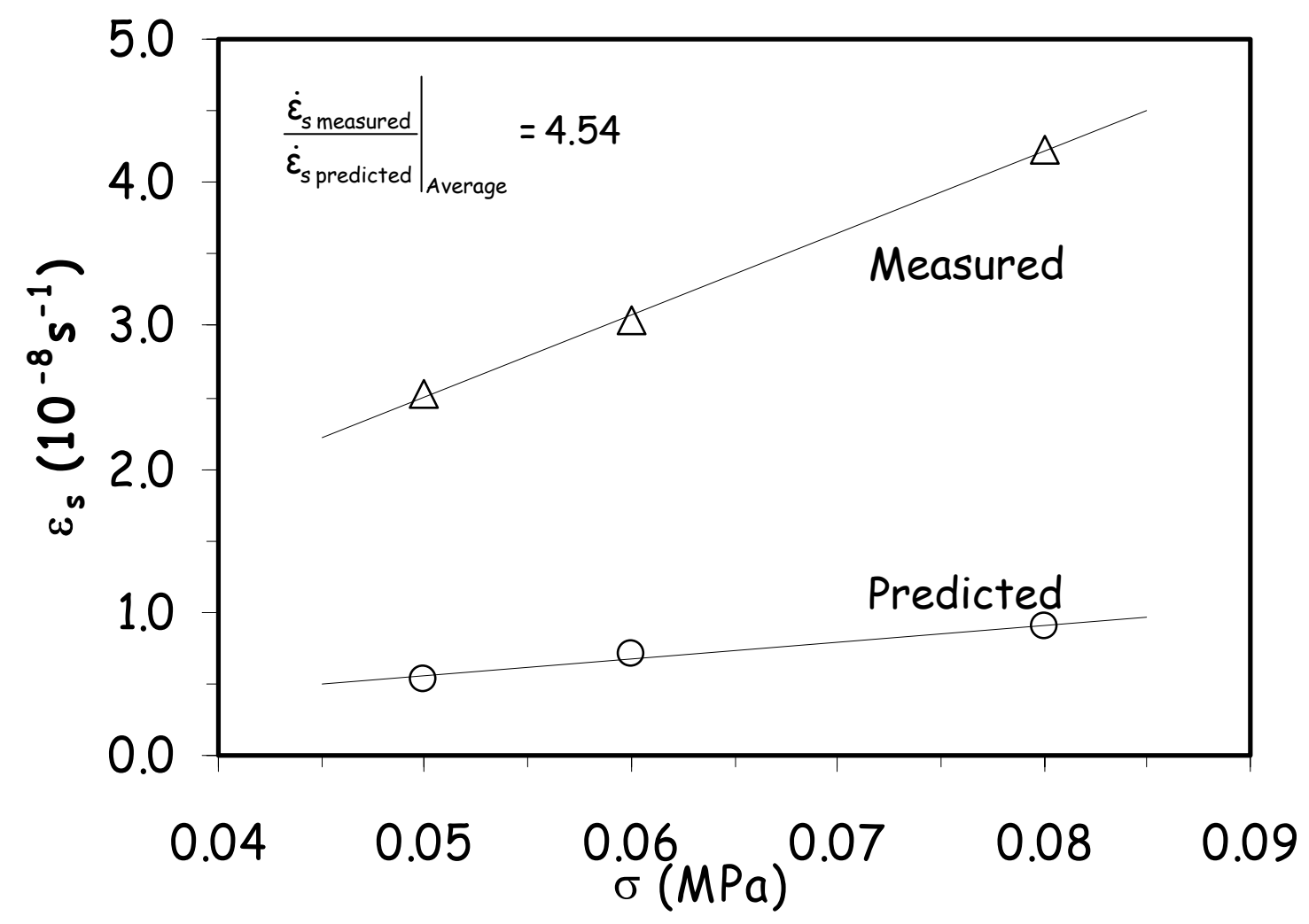

Figure 25. Comparison of the measured and predicted Harper-Dorn creep rates for steady-state for pure aluminum tested at $920 \mathrm{~K}$. The predicted creep rates were obtained from static annealing data using creep rate equation of the dislocation network theory of Harper-Dorn creep.

\section{b. HCP and BCC metals}

Our original model hcp material was $\mathrm{Mg}-0.5 \mathrm{wt} . \% \mathrm{Zr}$ alloy. During initial stages of the program we have developed procedures for annealing this alloy to obtain $1-2 \mathrm{~mm}$ grain size and to obtain dislocation etch pits. Although initial etch pitting results were very encouraging, in subsequent investigations we were unable to establish one-to-one correspondence between etch pits and dislocations. The reason for that was presence of extraneous pits associated not with dislocations but most likely with $\mathrm{ZrH}_{2}$ precipitates. These precipitates form readily during high temperature annealing when minute amount of moisture in annealing atmosphere can react with magnesium forming magnesium 
oxide and freeing hydrogen which subsequently reacts with zirconium (Squires, et al 1963). Despite numerous attempts to anneal samples in very pure argon gas atmosphere we were unable to eliminate this problem. As a resulted we decided to use pure $\mathrm{Zn}$ as an alternative model hep material. The suitable $12.7 \mathrm{~mm}$ diameter $99.99+\%$ pure zinc rod has been purchased from Alfa Aesar. The samples cut from that rod were annealed in argon for $24 \mathrm{hrs}$ at $673 \mathrm{~K}\left(0.95 \mathrm{~T}_{\text {melting }}\right)$ which resulted in recrystallization and grain size in the 1-2 mm range suitable for our studies. The procedure used for etching was the one developed by Brandt et al. 1963. It involves initial chemical polishing for 20 to $100 \mathrm{~s}$ in the solution of $160 \mathrm{~g} \mathrm{CrO}_{3}$ and $20 \mathrm{~g} \mathrm{Na}_{2} \mathrm{SO}_{4}$ in $500 \mathrm{ml}$ of distilled water (Solution 1) followed by dipping for 5-6 $\mathrm{s}$ in the solution of $1 \mathrm{~g} \mathrm{Hg}\left(\mathrm{NO}_{3}\right)_{2}$ in $1 \mathrm{ml} \mathrm{HNO}_{3}$ and $500 \mathrm{ml}$ of distilled water, dipping for 5-6 $\mathrm{s}$ in Solution 1, dipping for 2-3 $\mathrm{s}$ in the solution of 160 $\mathrm{g}$ of $\mathrm{CrO}_{3}$ in $500 \mathrm{ml}$ of distilled water, rinsing in water and finally drying in compressed air. The etching dissolves sample surface and forms hillocks at dislocations.

The annealed samples were crept in argon atmosphere in compression at the stress of $4 \mathrm{MPa}$ and temperature of $573 \mathrm{~K}$ to the steady state region and then cooled under load to room temperature. The creep apparatus used was the same one as for the aluminum.

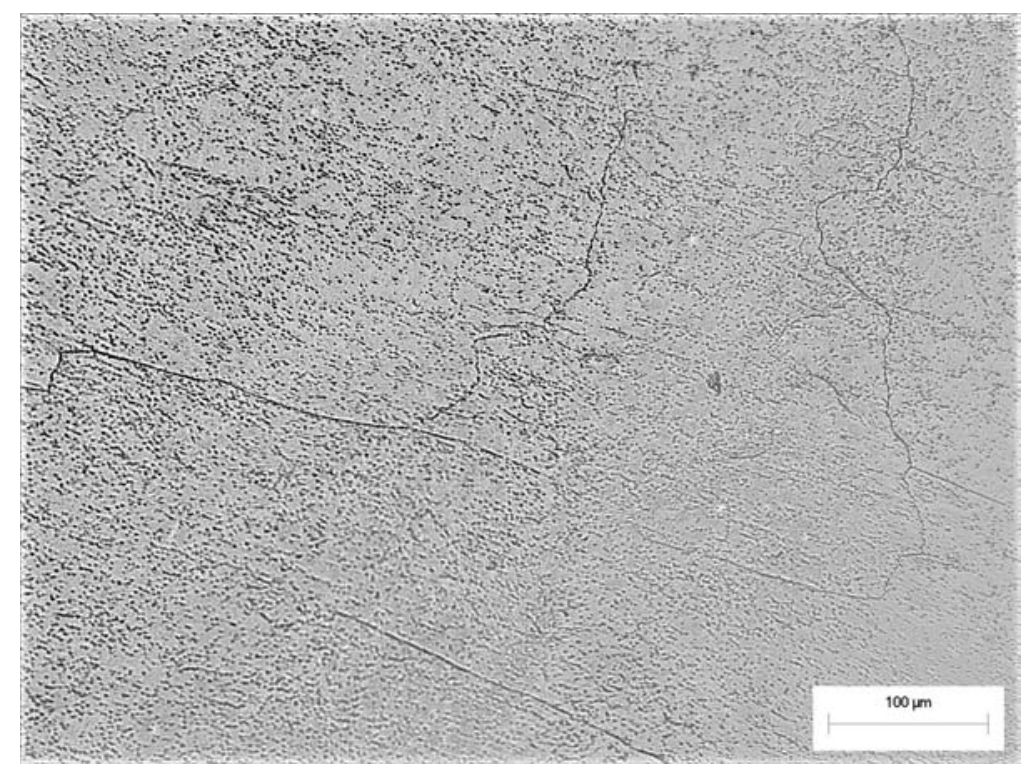

Figure 26. Dislocation structure of pure zinc sample after creep at $573 \mathrm{~K}$ in compression at constant stress of $4 \mathrm{MPa}$ to steady-state and subsequently cooled under load to room temperature. Density of dislocations not associated with boundaries is $\sim 1.6 \times 10^{5} \mathrm{~mm}^{-2}$. 


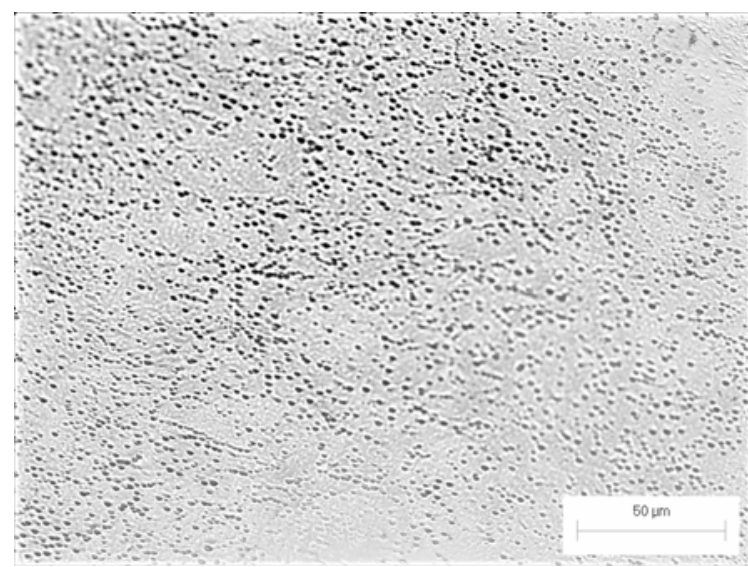

(a)

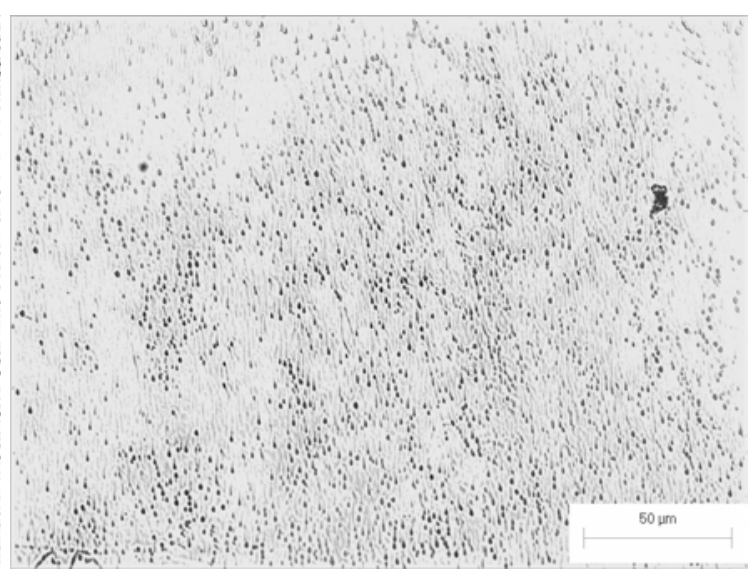

(b)

Figure 27. Dislocation structure of pure zinc sample (a) after creep at $573 \mathrm{~K}$ in compression at constant stress of $4 \mathrm{MPa}$ to steady-state and cooling under load to room temperature $\left(\rho=1.58 \times 10^{5} \mathrm{~mm}^{-2}\right)$ and (b) subsequent annealing for $2 \mathrm{~min}$ at the same temperature $\left(\rho=0.88 \times 10^{5} \mathrm{~mm}^{-2}\right)$.

Typical dislocation structure after loading is shown in Figure 26 and, at higher magnification, in Figure 27a. The micrographs show expected dislocation subgrain structure with dislocation density inside subgrains on the order of $\sim 1.6 \times 10^{5} \mathrm{~mm}^{-2}$. After subsequent annealing at $573 \mathrm{~K}$ all samples recrystallized. The recrystallization was not complete for samples annealed for up to 5 minutes which allow for measurements of dislocation densities in a few unrecrystallized grains. It is important to point out that this was not an easy task as only grains with orientations close to [0001] and [10 $\overline{10}]$ develop hillocks. Typical dislocation structure in unrecrystallized grains is shown in Figure $27 \mathrm{~b}$. The dislocation density is only about half of that of the as loaded samples and most of the subgrains dissolved. The dislocation density data obtained from unrecrystallized grains for samples annealed for 2 and 5 minutes are summarized in Table 3. For comparison the table also includes the data on dislocation structure for the recystallized grain with corresponding dislocation structure shown in Figure 28.

Although the obtain results were insufficient to asses annealing kinetics, they were more than adequate for obtaining reliable dislocation link length distributions. The 
Table 3. Characteristics of the dislocation structure of pure zinc deformed by creep at $573 \mathrm{~K}$ to steady state

\begin{tabular}{ccccc}
\hline & After loading & $\begin{array}{c}\text { Annealing for } \\
2 \text { min }\end{array}$ & $\begin{array}{c}\text { Annealed for } \\
5 \mathrm{~min}\end{array}$ & $\begin{array}{c}\text { Recrystallized } \\
\text { structure }\end{array}$ \\
\hline$\rho\left[\mathrm{mm}^{-2}\right]$ & $1.58 \times 10^{5}$ & $8.86 \times 10^{4}$ & $7.28 \times 10^{4}$ & $2.01 \times 10^{4}$ \\
\hline $\mathrm{N}\left[\mathrm{mm}^{-3}\right]$ & $7.04 \times 10^{7}$ & $3.2 \times 10^{7}$ & $2.36 \times 10^{7}$ & $0.32 \times 10^{7}$ \\
\hline$\langle\mathrm{L}\rangle[\mu \mathrm{m}]$ & 2.24 & 2.75 & 3.08 & 6.17 \\
\hline $\mathrm{L}^{*}[\mu \mathrm{m}]$ & 0.995 & 1.17 & 1.29 & 2.75 \\
\hline$\langle\mathrm{u}\rangle$ & 2.25 & 2.35 & 2.39 & 2.24 \\
\hline $\mathrm{m}$ & 1.578 & 1.65 & 0.999 & 1.188 \\
\hline
\end{tabular}

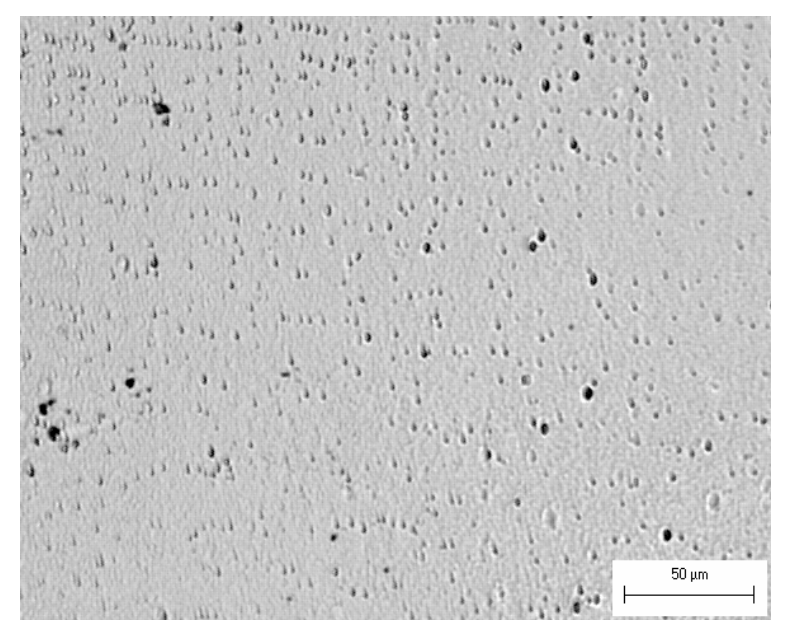

Figure 28. Dislocation structure in a recrystallized grain of pure zinc sample after creep at $573 \mathrm{~K}$ in compression at constant stress of $4 \mathrm{MPa}$ to steady-state, cooling under load to room temperature subsequent annealing for $5 \mathrm{~min}$ at the same temperature $\left(\rho=0.2 \times 10^{5} \mathrm{~mm}^{-2}\right)$.

experimental distribution are shown in Figure 29. All zinc distributions, including the one for the recrystalized structure, are similar suggesting that they can be represented by one universal curve. For comparison the figure also contains data points for the link length distribution for steady-state H-D creep for Al representing time invariant universal distribution for fcc metals. It appears that the differences between zinc and aluminum 
distributions are small and to the first approximation it could be assumed that they can be represented by one universal curve.

As for aluminum, the initial portion of the distributions were fitted to the function $\phi(L)=C \cdot L^{m}$ to obtain exponent, $m$, in our link growth law, $g(L, t)$, Figure 30 . The average value of $m$ is $1.244 \pm 0.30$, which is in good agreement with the value of $m=$ 1.33 obtained during Harper-Dorn creep of Al. This suggests that the kinetics of static

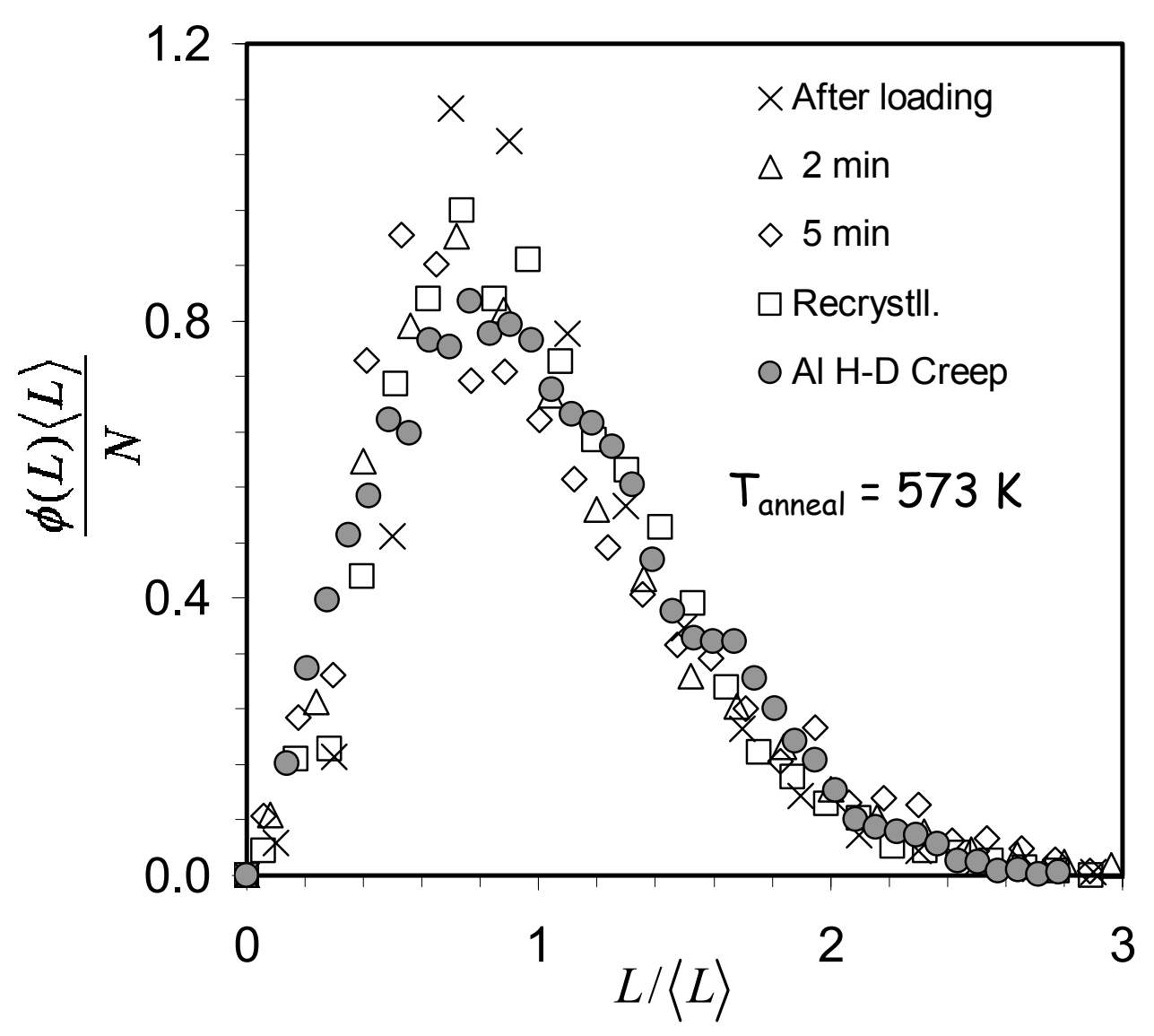

Figure 29. Experimental results showing the normalized dislocation link length distributions for pure zinc samples after creep at $573 \mathrm{~K}$ in compression at constant stress of $4 \mathrm{MPa}$ to steady-state and subsequently unloaded and statically annealed at the same temperature for the indicated times. Square data points are for dislocation structure in a recystallized zinc grain and dark circles are for the distribution obtained for aluminum after Harper-Dorn creep at temperature $913 \mathrm{~K}$ the constant stress of $0.08 \mathrm{MPa}$ to steady-state. 
annealing and coarsening of the dislocation networks during Harper-Dorn creep of zinc is similar to that of aluminum and that it can be described by the same growth law. We also not note that although the general shape of the distribution for recrystallized structure is similar to those after annealing, Figure 30, they differ especially in the short link region. This difference could be significant because it suggests smaller dislocation annihilation rate for the former.

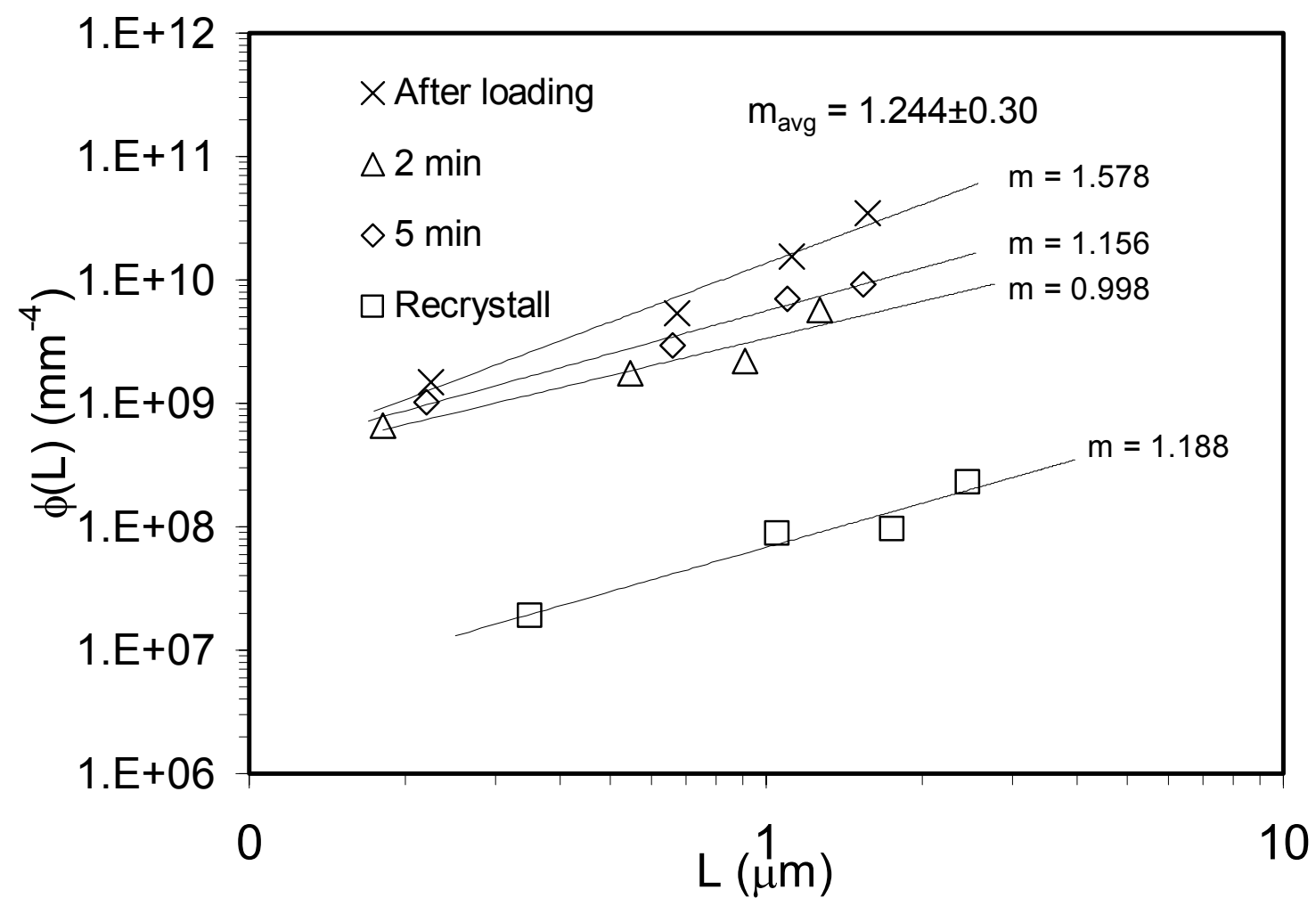

Figure 30. Experimental results showing a fit to the initial portion of the distributions shown in Figure 29 to obtain the exponent $m$ in the link growth law. All results are for pure zinc samples after creep at $573 \mathrm{~K}$ in compression at constant stress of $4 \mathrm{MPa}$ to steady state and subsequently unloaded and statically annealed at the same temperature for the indicated times. 
Because of the problems associated with obtaining dislocation etch pits in the $\mathrm{Mg}$ $-0.5 \mathrm{wt} . \% \mathrm{Zr}$, it was also decided to use white tin rather that $\mathrm{Mg}-11 \mathrm{wt} \% \mathrm{Li}$ as the model bcc material. White tin has a body centered tetragonal structure and eleven possible slip systems resulting from the combinations of five closed packed planes and four slip directions (Honda, 1978). Its creep behavior was studied by Mohamed et al 1973 who showed that at $495 \mathrm{~K}$ white tin exhibits H-D creep at stresses smaller than about 0.3 $\mathrm{MPa}$. The etching procedure to obtain dislocation structure in tin has been also known (Honda et al., 1972). It is a two-step process consisting of chemical polishing for one hour in the solution of 1 part of nitric acid, 1 part of acetic acid and 4 parts of glycerin followed by etching for 30 to $270 \mathrm{~s}$ in a $500 \mathrm{ml}$ solution consisting of $50 \mathrm{cc} \mathrm{HCl}, 63 \mathrm{~g}$ of $\mathrm{NH}_{4} \mathrm{NO}_{3}, 5 \times 10^{-3} \mathrm{~g}$ of $\mathrm{CuSO}_{4} \cdot 5 \mathrm{H}_{2} \mathrm{O}$ and distilled water. The samples used in the annealing studies of tin have been cut from $12.7 \mathrm{~mm}$ diameter $99.98 \%$ tin rod purchased from Alfa Aesar. The samples were annealed for $22 \mathrm{hrs}$ at $478 \mathrm{~K}$ which resulted in the completely recrystallized structure with 2 to $4 \mathrm{~mm}$ grain size. The annealed samples were then

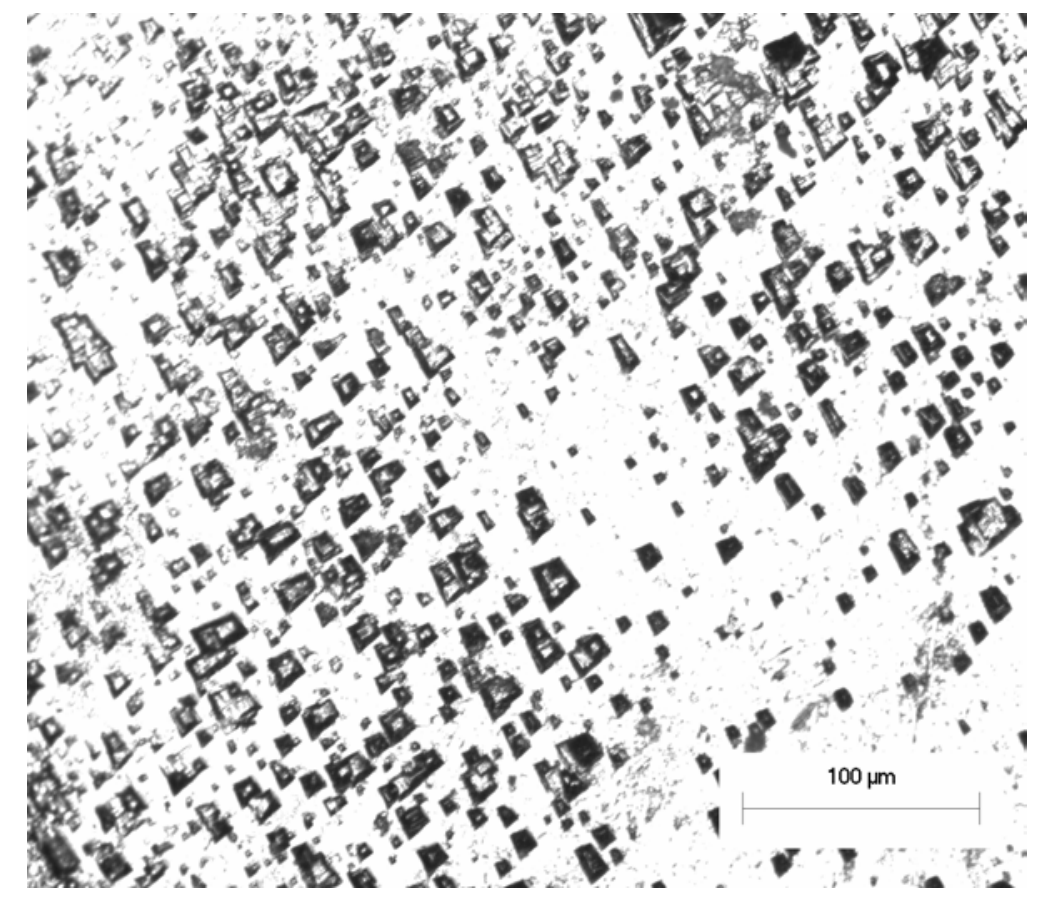

Figure 31. Dislocation etch pit structure of pure tin sample after creep at $423 \mathrm{~K}$ in compression at constant stress of $2 \mathrm{MPa}$ to steady-state and cooling under load to room temperature; dislocation density $\rho=9,803 \mathrm{~mm}^{-2}$. 
deformed in compression with the constant stress of $2 \mathrm{MPa}$ at $423 \mathrm{~K}$ to steady state and then cooled under load to room temperature. All subsequent annealing for the times ranging from $15 \mathrm{~s}$ to $10 \mathrm{~min}$ were also done at $423 \mathrm{~K}$ and resulted in partially tocompletely recrystallized structure. Despite numerous attempts we were unable to obtain sufficiently clear etch pit that would allow us to characterize the annealed dislocation structure. We were however successful in obtaining clear pits in the as deformed sample. The obtained structure is shown in Figure 31 and the results of the characterizations are summarized in Table 4. Figure 32 shows the resultant link length

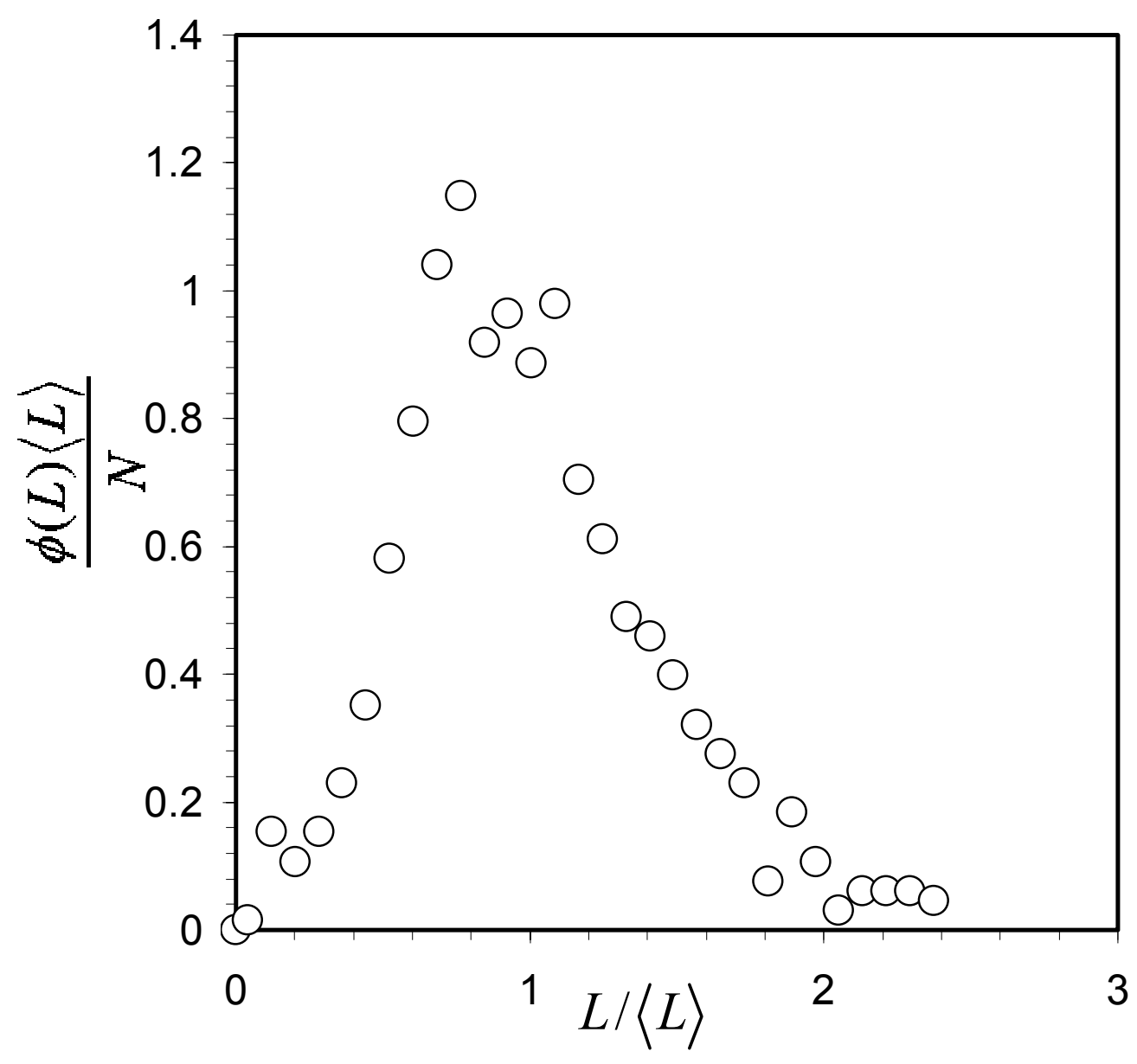

Figure 32. Experimental results showing the normalized dislocation link length distributions for pure tin samples after creep at $423 \mathrm{~K}$ in compression at constant stress of $2 \mathrm{MPa}$ to steady-state and subsequently cooled after load to room temperature 
Table 4. Characteristics of the dislocation structure of pure tin deformed by creep at $423 \mathrm{~K}$ to steady state

\begin{tabular}{cc}
\hline$\rho$ & $9,803 \mathrm{~mm}^{-2}$ \\
\hline $\mathrm{N}$ & $1.12 \times 10^{6} \mathrm{~mm}^{-3}$ \\
\hline$\langle\mathrm{L}\rangle$ & $8.82 \mu \mathrm{m}$ \\
\hline $\mathrm{L}_{\text {star }}$ & $4.01 \mu \mathrm{m}$ \\
\hline$\langle\mathrm{u}\rangle$ & 2.20 \\
\hline $\mathrm{m}$ & 1.162 \\
\hline
\end{tabular}

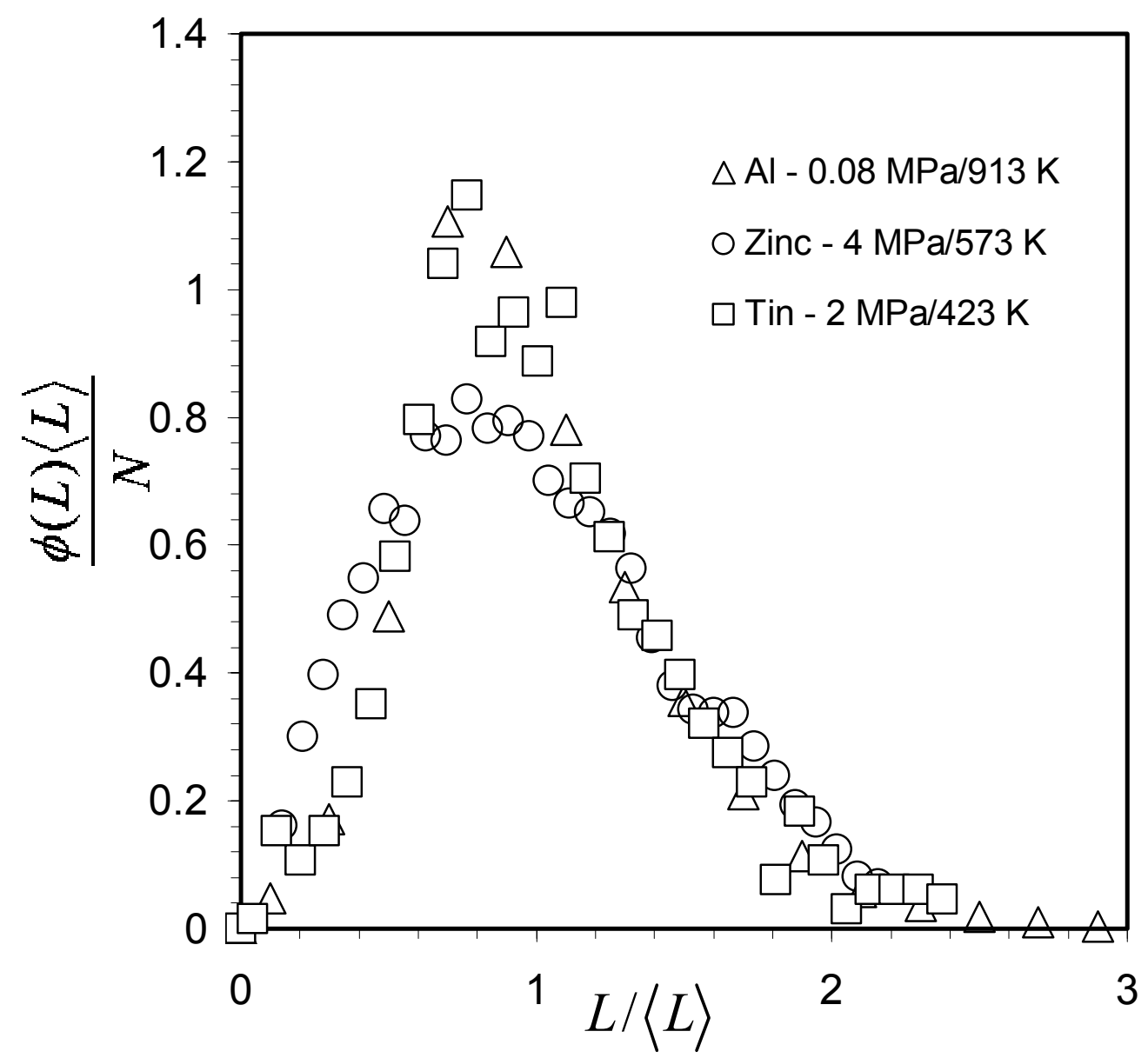

Figure 33. Experimental results showing comparison of the normalized dislocation link length distributions for pure aluminum, zinc and tin samples after creep to steady state at indicated temperatures and stresses. 
distribution. The initial slope of that distribution is 1.162 which is not very different from the values obtained for aluminum and zinc.

Figure 33 compares link length distributions for the as all three investigated materials for samples after creep test. The three distributions are quite similar in the long link region but slightly diverge at the lower link length. The difference is the largest for zinc which could be a result of the limited number of the available slip system for that material hence smaller variety of dislocations with different Burgers vectors. These results suggest that the scaled link length distribution is universal and the same for the three considered crystal structures. This supports our theoretical findings that appropriately scaled dislocation link length distribution should both universal and time invariant. 


\section{$\underline{\text { References }}$}

Ardell, A. J., Reiss, H., and Nix, W. D., 1965, J. Appl. Phys., 36, p. 1727.

Ardell, A. J., and Przystupa, M. A., 1984, Mechanics of Materials, 3, pp. 319-332

Ardell, A. J., and Lee, S. S., 1986, Acta Metall., 34, pp. 2411-2423.

Ardell, A. J., and Przystupa, M.A., 1993, High Temp. Def'n and Processing, 12, pp. 1-11.

Ardell, A. J., 1997, Acta Metall., 45, pp. 2971-2981.

Barrett, C. R., and Nix, W. D., 1965, Acta Metall., 13, pp. 1247-1258.

Barrett, C. R., 1967, Trans. Metall. Soc. AIME, 239, pp. 1724-1729.

Barrett, C. R., Muehleisen, E. C., and Nix, W. D., 1972, Mater. Sci. Engng,. 10, 33.

Banerdt, W. B., and Sammis, C. G., 1985, Phys. Earth Planet. Interiors, 41, pp. 108-124

Berbon, M.Z., Owen, D.M., and Langdon, T.G., 1996, The Johannes Weertman Symposium, ed. by R.J. Arsenault et al., TMS, Warrendale, PA, pp. 145-154.

Blum, W., and Maier, W., 1999, Phys. Stat. Sol., 171, pp. 467- 474.

Brandt, R. C., Adams, K. H., and Vreeland, Jr., T., 1963, Journal of Applied Physics, 34, pp. 587-590.

Cadek, J., and Fiala, J., 1988, Acta Technica CSAV, no. 1, pp. 1-13.

Daily, S., and Ahlquist, C. N., 1972, Scripta Metallurgica, 6, pp. 95-100.

Delgado Friedrichs, O., O’Keeffe, M., and Yaghi, O. M., 2003, Acta Crystollagraphy, vol. A59, pp. 22-27

Evans, H. E., and Knowles, G., 1977, Acta Metall., 25, pp. 963-975.

Frank, F.C., 1950, "The Origin of Dislocations," Symposium on Plastic Deformation of crystalline Solids, Carnegie Inst. Tech. and Dept. of the Navy, ONR, Washington, DC (1950), p. 100.

Friedel, J., Dislocations, 1964, Pergamon Press, Oxford.

Frost, H. J., and Ashby, M. F., 1982, Desformation-Mechanism Maps, Pergamon Press, Oxford, United Kingdom.

Garafano, F., 1963, Trans. Metall. Soc. AIME, 227, p. 351.

Ginter, T.J., Chaudhury, P.K., and Mohamed, F.A., 2001, Acta Mater., 49, pp. 263-272.

Ham, R., and Sharpe, N., 1961, Phil. Mag., 6, p. 1193

Harper, J., and Dorn, J.E., 1957, Acta metall., 5, p. 654.

Harper, J. G., Shepard L. A., and Dorn, J. E., 1958, Acta Metall., 6, pp. 509-518. 
Herring, C., 1950, J. appl Phys., 21, p. 437.

Hillert, M., 1965, Acta Metall., 13, pp. 227-238.

Hirth, J.P., and Lothe, J., 1968, Theory of Dislcoations, McGraw-Hill, New York.

Honda, K., and Hirokawa, T., 1972, Japanese Journal of Applied Physics, 12, pp. 17631774

Honda, K., 1978, Japanese Journal of Applied Physics, 17, pp. 33-41.

Jones, H., and Leak, G. M., 1966, Acta Metall., 14, pp. 21-27.

Justice, Jr., M.G., Graham,E.K., Tressler, R.E., and Tsong, I.S.T., 1982, Geophys. Res. Lett., 9, pp. 1005-1008.

Kassner, M.E., and Pérez-Prado, M.-T., 2000, Prog. Mater. Sci., 45, pp. 1-102.

Kassner, M. E., Kumar, P., and Blum, W., 2007, Int. J. Plasticity, 23, pp. 980-1000.

Kumar, P., Kassner, M. E., and Langdon, T. G., 2007, J. Mater. Sci., 42, pp. 409-420

Kohlstedt, D.L., and Goetze, C.,1974, J. of Geophys. Res., 1974, 79, pp. 2045-2051.

Lagneborg, R., 1972, Met. Sci. J., 6, pp. 127-133.

Lagneborg, R., and Forsen, B.-H., 1973, Acta Metall., 21, p. 781-790.

Lagneborg, R., Forsen, B.-H., and Wiberg, J, 1974, Creep Strength in Steel and High-

Temperature Alloys, The Metals Society, London, , pp. 1-7.

Langdon, T. G., and Yavari, P., 1982, Acta Metall., 30, pp. 881-887

Lee, S. S., 1985, Ph. D. Dissertation, UCLA, Los Angeles, CA.

Li, J. C. M., 1966; Recrystallization, Grain Growth and Texture, American Society for Metals, Metals Park, Ohio, p. 45.

Lifshitz, I.M., and Slyozov, V.V., 1959, Soviet Physics JETP, 35, pp. 331-339.

Lin, P., Przystupa, M. A., and Ardell, A. J., 1985, "Dislocation Network Dynamics During Creep Deformation of Monocrystalline Sodium Chloride" in Strength of Metals and Alloys, ICSMA-7, edited by H. K. McQueen et al. (Pergamon Press, Oxford, 1985), p. 595

Lin, P., Lee, S. S., and Ardell, A. J., 1989, Acta Met., 37, pp. 739-748.

Malakondaiah, G., and Roma Rao, P., 1979, Scripta Met., 13, pp. 1197-1190.

Malakondaiah, G., and Roma Rao, P., 1982, Acta Met., 29, p. 1263.

Miekk-Oja, H. M., and Lindroos, V. K., 1972, Surface Science, 31, pp. 422-455

Mitra, S.K., and McLean, D., 1967, Metal Sci. J., 1, pp. 192-198. 
Mohamed, F. A., and Wolfenstine, J., 1991, Hot Deformation of Aluminum Alloys, ed. T. G. Langdon, H. D. Merchant, J. G. Morris and M. A. Zaidi, TMS, pp. 223-237.

Mohamed, F. M., Murty, K. L., and Morris, J. W., 1973, Metal. Trans., 4, p. 935

Mohamed, F. M., Murty, K. L., and Morris, J. W. , 1975, Rate processes in Plastic Deformation of Materials, ed. J. C. M. Li and A. K. Mukherjee, ASM, Metal Park, Ohio, p. 459.

Mohamed, F.A., and Ginter, T.J., 1982, Acta Metall., 30, pp. 1869-1881

Nabarro, F.R.N., 1948, Report of a Conference on Strength of Solids, p. 75. Physical Soc., London.

Nabarro, F. R. N., 1989, Acta metall., 37, pp. 2217-22

Nabarro, F. R. N., 1997, Phil. Mag., 1971, 75, pp. 703-711.

Nabarro, F. R. N., 1997a, Mater. Sci. Engng,. A234-236, pp. 67-76.

Nabarro, F.R.N., 2000, Phys. Stat. Sol., 182, pp. 627- 629.

Nabarro, F.R.N., 2000a, Phys. of Sol. State, 42, pp. 1456- 1459.

Ness, E., Progress in Material Science, 1998, 41, pp. 129-193.

Ness, E., Marthinsen, K., and Roning, B., 2001, J. of Mater. Proc. Tech.. 117, 333-340.

Ness, E., Blum, W., and Eisenlohr, P., 2002, Metall. and Mater. Trans. A, 33A, 305-310.

Ness, E., and Marthinsen, K.,2002a, Mater. Sci. Engng,.A322, 176-193.

Ness, E., Marthinsen, K., and Brechet, Y., 2002b, Scripta Met., 47, pp. 607-611.

Nix, W. D., Gasca-Neri, R., and Hirth, J. P., Phil. Mag., 1971, 23, pp. 1339-1349.

Nost, B., and Nes, E., 1969, Acta Metall., 17, pp. 13-20.

Novotny, J., Fiala, J., and Cadek, J., 1983, Acta Metall., 31, p. 1697.

Novotny, J., Fiala, J., and Cadek, J., 1985, Acta Metall., 33, pp. 905-911.

Oden, A., Lind, E., and Lagneborg, R., 1974, Creep Strength of Steel and HighTemperature Alloys, Metal Society, London, p. 60

Ostrom, P., and Lagneborg, R., 1976, J. Engineer. Mater. Tech., Trans. ASME, Ser. H, 98, pp.114-124.

Ostrom, P., and Lagneborg, R., 1980, Res Mech., 1, p. 59-79.

Owen, D. M., and Langdon, T. G., 1996, Mater. Sci. Eng., A216, pp.20-29. 
Przystupa, M. A., and Ardell, A. J., 2000, Deformation, Processing, and Properties of Structural Materials, ed. E. M. Taleff, C. K. Syn, and D. R. Lesuer, The Mineral, Metal \& Mateials Society, pp. 157-168.

Raman, V., and Raj, S. V., 1985, Scripta Met., 19, pp. 629-634.

Ramesh, K. S., Yasuda, E., and Kimura, S., 1986, J. Mater. Sci., 21, pp. 4015-4018.

Ramesh, K. S., Yasuda, E., Kimura, S., and Urabe K.,1986a, J. Mater. Sci., 21, pp. 3147-3152.

Raj, S. V., 1985, Scripta Met., 96, pp. 57-64.

Raj, S. V., 1987, Mater. Sci. Eng., A216, pp.20-29.

Ruano, O. A., Wadsworth, J., and Sherby, O. D., 1988, Acta Metall., 36, pp. 1117-1128.

Ruano, O. A., Wadsworth, J., and Sherby, O. D., 1988a, Scripta Metall., 22, pp. 19071910.

Ruano, O. A., Wolfenstine, J., Wadsworth, J., and Sherby, O. D., 1992, J. Am. Ceram. Soc., 75, pp. 1737-1741.

Sargent, P. M., and Ashby, M. F., 1982, Scipta Met., 16, pp. 1415-1422.

Seeger, A., Kronmuller, H., Mader, S., and Trauble, H., 1961, Phil. Mag., 6, p. 639.

Squires, R. L.,. Weiner, R. T, and Phillips, M., 1963, Journal of Nuclear Materials,. 8, pp. 77-80.

Stang, R. G., Nix, W. D., Barrett, C. R., 1973, Metall. Trans., 4, pp. 1695-1699.

Turunen, M. J., and Lindroos, V. K., 1974, Phil. Mag., 29, pp. 701-708.

Turunen, M. J., and Lindroos, V. K., 1973, Phil. Mag., 27, pp. 81-86.

Turunen, M. J., 1976, Acta Metall., 24, pp. 463-467.

Underwood, E. E., 1970, "Quantitative Stereology”, Addison-Wesley Publishing Company, Inc, Philadelphia, 1970

Wagner, C., 1961, Z. Electrochem., 65, p. 581.

Wang, J. N., and Langdon, T. G. , 1994, Acta metal. mater., 42, pp. 2487-2492.

Wang, J. N., Mater Sc and Eng A., 1995, A202, pp. 52-56.

Wang, J. N., Acta Mater., 1996, 44, pp. 855-862.

Wang, J. N., Mater Sc and Eng A., 1996a, A206, pp. 259-269.

Weertman, J., 1968, Trans. of ASM, 61, pp. 681-694.

Weertman, J., and Blacic, J., 1984, Geophys. Res. Letters, 11, pp. 117-120. 
Wu, M. Y., and Sherby, O. D., 1984, Acta Metall., v. 32, pp. 1561-1572.

Yavari, P., Miller, D. A., and Langdon, T. G., 1982, Acta Metall., 30, pp. 881-887. 
Appendix 1. Publications, Presentations and Personnel

In addition to three annual progress reports, the research on this project resulted in the following publications and presentations:

Publications:

1. Y. Tan, M. A. Przystupa and A. J. Ardell, "Evolution of dislocation link-length distributions during static annealing", Proceedings of the 2nd International Conference on Multiscale Modeling of Materials (MMM-2), edited by N. M. Ghoniem, UCLA, Los Angeles, CA, 2004, p. 298.

2. Y. Tan, M. A. Przystupa and A. J. Ardell, "Evolution of the Dislocation Link-Length Distribution During Static Annealing of Pure Aluminum Polycrystals”, in preparation.

3. M. A. Przystupa, "Characterization of the Dislocation Link-Length Distributions Using Data from the Dislocation Etch-Pit Patterns", in preparation.

Presentations:

1. Y. Tan, M. A. Przystupa and A. J. Ardell, "Evolution of dislocation link-length distributions during static annealing", $2^{\text {nd }}$ International Conference on Multiscale Modeling of Materials (MMM-2), Los Angeles, CA, Oct. 11-15, 2004.

2. M. A. Przystupa, Y. Tan and A. J. Ardell, "Predicting Harper-Dorn creep rates using data on static annealing”, TMS $134^{\text {th }}$ Annual Meeting and Exhibition, San Francisco, CA, Feb. 13-17, 2005.

3. M. A. Przystupa, Y. Tan and A. J. Ardell, "Harper-Dorn Creep of fcc, bcc and hcp Metals and Alloys”, 2006 DOE/BES Mechanical Behavior Contractors Meeting, San Antonio, TX, March 15-17, 2006

Personnel:

The following individuals received either total or partial financial support on this project:

Professor Marek A. Przystupa, Principal Investigator;

Professor Alan J. Ardell, co-Principal Investigator; 
Dr. Yi Tan, post-doctoral researcher; currently Professor Dalian University of Technology, China;

Mr. Evan Epstein, undergraduate laboratory helper;

Mr. Manan Mehta, undergraduate laboratory helper;

Mr. Malek Kunbargi, undergraduate laboratory helper;

Mr. Jonathan E. Sunio, undergraduate laboratory helper;

Mr. Grant M. Kao, undergraduate laboratory helper. 
Appendix 2. Compilation of useful relationships

The following equations are identities used throughout this report during derivations of the relations describing evolutions of the link length distributions.

$$
\begin{aligned}
& \int_{0}^{L_{c}} \frac{\partial(\phi g)}{\partial l} d L=-\lim _{L \rightarrow 0}(\phi g) \\
& \int_{0}^{L_{c}} L \frac{\partial(\phi g)}{\partial l} d L=-N\langle g\rangle \\
& \int_{0}^{L_{c}}\left(\int_{L}^{L_{c}} \frac{\phi\left(L^{\prime}\right)}{\partial L^{\prime}} d L^{\prime}\right) d L=N_{n} \\
& \int_{0}^{L_{c}} L\left(\int_{L}^{L_{c}} \frac{\phi\left(L^{\prime}\right)}{\partial L^{\prime}} d L^{\prime}\right) d L=\frac{1}{2} N_{n}\left\langle L_{n}\right\rangle \\
& \frac{d}{d L}\left(\int_{L}^{L_{c}} \frac{\phi\left(L^{\prime}\right)}{\partial L^{\prime}} d L^{\prime}\right)=-\frac{\phi(L)}{L} \\
& \int_{0}^{L}\left(\int_{L^{\prime}}^{L_{m}} \frac{\phi\left(L^{\prime \prime}\right)}{\partial L^{\prime \prime}} d L^{\prime \prime}\right) d L^{\prime}=L \int_{L}^{L_{m}} \frac{\phi\left(L^{\prime}\right)}{\partial L^{\prime}} d L^{\prime}+\int_{0}^{L} \phi(L) d L
\end{aligned}
$$

